\title{
Dynamics, ionization and charge separation in superheated metastable water
}

\author{
Dissertation \\ zur Erlangung des Doktorgrades \\ der Mathematisch-Naturwissenschaftlichen Fakultäten \\ der Georg-August-Universität zu Göttingen
}

vorgelegt von

Esteban Vöhringer-Martinez

aus Córdoba, Argentinien

Göttingen 2008 
D7

Referent: Prof. Dr. Jürgen Troe

Korreferent: Prof. Dr. Helmut Grubmüller

Tag der mündlichen Prüfung: 2. Juli 2008 


\section{Acknowledgements}

I would like to express my deep and sincere gratitude to my supervisor Prof. Abel for his guidance over the past four years and Prof. Grubmüller for the fruitful discussions and contributions to my work.

I wish to express my warm thanks to Prof. Troe for hosting me in his group at the beginning of my thesis and Prof. Botschwina for his help in questions related to quantum mechanics.

I am very grateful to Gerrit "The Master" Groenhof, who introduced me into the world of computer simulations and shared the room with me, the stinky "gaucho".

I warmly thank Frank Wiederschein for his tireless help trying to obtain the distribution we were looking for and for the discussions where I learned a lot. Frank, Jürgen Haas, and Dirk Matthes, I would also like to thank for reading the awful english of the first drafts and giving me great tips on how to write what I wanted to express.

My sincere thanks are due to the whole Grubmüller group, which always provided me with valuable advice concerning the simulations. I wish to also thank the Abel group and especially Oli, Kathrin, Yiaxing, Ales, and Alexander for explaining me the experimental difficulties and performing the experiments.

My biggest thanks go to my parents. Les agradezco de todo corazón por haberme posibilitado todo esto y por todo aquello que no se puede formular en palabras.

I owe my loving thanks to my family, my wife Ale and my daughter Lara. Ustedes representan para mi lo más importante en este mundo y les debo miles de gracia por darme lo que me dan todos los días y en especial en este último tiempo. 



\section{Summary}

Heating up a water filament in vacuum with an Infrared(IR)-laser generates superheated metastable water, which undergoes an ultrafast phase expansion.

Here, Molecular Dynamics (MD) simulations were used to follow this process and to provide a molecular picture for recent experiments using photoelectron spectroscopy. From the experiments, time scales on the order of hundreds of picoseconds were assigned for the evolution of the metastable phase, which depend on the initial temperature. It was found that methanol under comparable conditions was slower and showed different characteristics in the spectra.

From the simulations two different temperature regimes for the evolution of metastable water could be identified: a subcritical and a supercritical regime. At subcritical initial temperatures the process takes place as single molecule evaporation from the liquid-vacuum interface. At supercritical temperatures, however, the phase evolves much faster $(\sim 100 \mathrm{ps})$. The simulation results showed that the evolution of characteristic bands in the spectrum is accompanied with changes in the nature of the hydrogen bonds. Aggregates of 5-20 water molecules built during the simulations identified a new band appearing in the photoelectron spectrum after $200 \mathrm{ps}$.

In accordance with the measured spectra, the simulations of methanol revealed much slower dynamics than water and no aggregates were built. The difference between the two hydrogen bonded liquids stems from slower dynamics in the formation and dissociation of hydrogen bonds in methanol, which represent the first step in the evolution of these metastable phases. Furthermore, QM/MM-calculation assigned shifts in supercritical water spectra to a destabilization of the ground and the final state of the ionization process due to hydrogen bonds.

An IR-laser is also used to isolate ions or charged molecules from a liquid 
water filament in mass spectrometric analysis.

MD-simulations of model systems, describing the water filament and the influence of the IR-laser, showed that shock waves divide the liquid matrix into smaller volumes. The minor increase in temperature in the divided volumes, revealed the softness of the isolation method, which allows the characterization of fragile proteins and protein-complexes.

In addition, the mechanism for the generation of charges during the dispersion of the liquid was studied. Three regimes were identified for the probability to obtain a charged volume after the shock waves, which were dependent on the ion concentration. The charge probability for the divided volumes at concentrations lower than $10^{-3} \mathrm{M}$ was derived from an analytical expression assuming a Poisson distribution for the number of ions in the volumes. For higher concentrations the ion-ion interactions had to be taken into account, which reduced this charge probability. In addition, a flux of ions during the separation of the liquid at concentrations larger than $10^{-1} \mathrm{M}$, required MD-simulations of the process. This flux increased with the charge of the divided volume at the beginning of the separation process and reduced the charge probability of the volumes even further. 


\section{Contents}

1 Introduction $\quad 1$

2 Methods $\quad 7$

2.1 Born-Oppenheimer Approximation . . . . . . . . 8

2.2 Classical Dynamics . . . . . . . . . . . . . . . 9

2.3 Molecular Dynamics . . . . . . . . . . . . . . . . . 10

2.4 Quantum Mechanics . . . . . . . . . . . . . . . 11

2.4.1 Hartree-Fock Method . . . . . . . . . . . . . . 13

2.4.2 Koopmans' Theorem . . . . . . . . . . . . 16

2.4 .3 Electron Correlation . . . . . . . . . . . . 17

2.5 Quantum Mechanics Molecular Mechanics Method . . . . . . 20

2.6 Empirical Force Fields . . . . . . . . . . . . . . . . . . . . 22

2.6.1 Water Models . . . . . . . . . . . . . . . 24

2.6.2 Methanol Models . . . . . . . . . . . . . 27

2.7 Hydrogen Bonds . . . . . . . . . . . . . . . . . . . . 28

2.7.1 Hydrogen Bond Kinetics . . . . . . . . . . . . . 29

3 Ultrafast Dynamics of Metastable Liquids 33

3.1 Experiment . . . . . . . . . . . . . . . . 33

3.2 Photoelectron Spectroscopy . . . . . . . . . . . . . 35

3.3 Simulation Setup . . . . . . . . . . . . . . . . . . 49

3.4 Results . . . . . . . . . . . . . . . . 50

3.4.1 Density ....................... 54 
3.4.2 Hydrogen Bonds . . . . . . . . . . . . . 56

3.4.3 Hydrogen Bond Dynamics . . . . . . . . . . . . . 59

3.4.4 Aggregates. . . . . . . . . . . . 60

3.4.5 Dynamics of Thermally Excited Water Aggregates: Dimer, Trimer, Tetramer . . . . . . . . . . . . 62

3.4.6 Simulated Photoelectron Spectra of Water . . . . . . 64

4 Laser Induced Isolation of Charges from Water 69

4.1 Experiment . . . . . . . . . . . . . . . . . . 69

4.2 Simulation Setup . . . . . . . . . . . . . . . . 72

4.2.1 Temperature Gradient . . . . . . . . . . . . 73

4.3 Results . . . . . . . . . . . . . . . 76

4.3 .1 Shock Waves . . . . . . . . . . . . 76

4.3.2 Ion Isolation through Ablation . . . . . . . . . . . 80

4.3.3 Isolation of Biomolecules from Liquid Water Matrix . 89

5 Discussion $\quad 91$

5.1 Ultrafast Dynamics in Metastable Liquids . . . . . . . . . . 91

5.2 Laser Induced Isolation of Charges from Water . . . . . . . . 95

$\begin{array}{lc}\text { List of Figures } & 103\end{array}$

$\begin{array}{ll}\text { List of Tables } & 105\end{array}$

$\begin{array}{ll}\text { Bibliography } & 107\end{array}$ 


\section{Chapter 1}

\section{Introduction}

Water is a chemical substance which is found throughout the universe in its three aggregate states: gas, liquid and solid. Water vapor is found in atmospheres of several planets like Mercury, Venus or Mars. Liquid water is suggested to be under the surface of Saturn's moon Enceladus and, furthermore, ice forms the polar caps on Mars and Titan. On earth, water covers $70 \%$ of its surface as oceans, it is present as vapor in clouds and also as ice at the polar caps. The interchange between the aggregate states is accompanied by heat transfer, which forms the basis for the heat transport from the equator to the northern and southern hemispheres.

Water constitutes 50-60\% of our body mass and plays a major role in biological processes like the photosynthesis. On a smaller scale, the dynamic structure and molecular properties of water are crucial for many chemical and biological processes: It serves as solvent for hydrophilic substances or as proton carrier for proton transfers between an acid and a base. Furthermore, single water molecules were found to catalyze radical-molecule reactions in the gas phase [1], which are relevant for the removal of atmospheric pollutants.

As a liquid, water exhibits anomalies distinguishing it from most other liquids. Compared to ammonia or hydrogen fluoride, water has a higher 
melting and boiling point. Its maximum density is at $4{ }^{\circ} \mathrm{C}$, well above its melting point, and the minimum in its isothermal compressibility is at $46^{\circ} \mathrm{C}$. These anomalies cause water to freeze "top down", which ensures life under frozen water surfaces. One reason for these anomalies is the formation of hydrogen bonds between the electronegative oxygen and the hydrogen atom. These interactions are ten times stronger than Van der Waals forces which dominate the interatomic interactions in most other liquids. The nature and the dynamics of these hydrogen bonds is the topic of ongoing investigations $[2,3,4]$.

Research on water represents a field of continuous strong interest in science also at extreme conditions $[5,6]$. If water is cooled to below its melting point, without impurities causing crystallization, a supercooled phase is reached. This phase reveals new thermodynamic properties, like divergent isothermal compressibility and heat capacity at $-45^{\circ} \mathrm{C}$ [6]. Water can also be heated up to above its critical point $\left(T_{\text {crit }}=374^{\circ} \mathrm{C}\right)$, where it forms a supercritical phase. At these conditions water features properties that can be quite different from those at standard conditions. It exhibits a higher solubility for organic compounds, a smaller one for salts, and also a higher degree of self ionization. These properties render supercritical water as an important agent in many areas ranging from high temperature chemistry [7] to hazardous waste treatment.

As the thermodynamic properties of water vary over a wide range of conditions, the underlying microscopic picture is still a subject of debate. X-ray absorption and X-ray Raman scattering experiments revealed details on the microscopic structure at standard [3] and at supercritical conditions [8], like the average number of hydrogen bonds and the coordination of a water molecule in the first solvation shell. Winter et al. [9] studied the local hydrogen bonding network at the liquid-water interface of a water filament in vacuum with photoelectron spectroscopy. They were able to 
identify characteristic hydrogen bonded configuration of water molecules at interfaces. Photoelectron spectroscopy represents a very sensitive technique used in static Electronic Spectroscopy for Chemical Analysis(ESCA) to display binding states of molecules [10]. Recently, photoelectron spectroscopy also has been shown to be suitable for studying dynamics with a high temporal resolution [11].

Although the two limiting cases represented by the supercritical phase and the liquid phase have been widely studied, very little is known on the transition from one phase to the other. This transition, and especially the time scale on which it occurs, is of great interest, as it is governed by characteristic features of the two phases.

Experimental work performed by Link [11] focused on this issue. A liquid water filament was heated to supercritical temperatures with an Infrared(IR)fs-laser and photoelectron spectra of the evolution of the superheated phase were recorded using a time resolved IR-XUV pump probe technique. The spectra obtained revealed characteristic shifts and assigned a time scale of several hundreds of picoseconds to the process depending on the deposited IR-energy. In contrast, the same experiments performed with methanol displayed much slower dynamics. These differences between the two liquids suggest molecular properties to be responsible for the observed dynamics. However, an assignment of spectral features on a molecular level was not possible.

Here, computer simulations were used to provide a microscopic picture of the evolution of the metastable phase and to correlate dynamics on a molecular level with the observed spectra. In Chapter 3 the experimental setup used to study the process as well as the main features of photoelectron spectroscopy on liquids will be presented. Furthermore, the simulation setup and the obtained simulation results will be compared to the experiment and a microscopic picture for the observed spectral shifts and bands will be given. The different behavior of water and methanol will be discussed in terms of hydrogen bond dynamics. 
Extreme conditions in liquid water also become accessible via shock waves. At the location of the shock front extreme thermodynamic conditions characterized by temperatures of several $1000{ }^{\circ} \mathrm{C}$ and densities twice as large as in the liquid phase can be reached. Shock waves travel through the liquid phase with a velocity larger than the velocity of sound, thereby inducing changes in thermodynamic properties on a very short time scale. Furthermore, shock waves play an important role in areas from detonation of explosives to astrophysics. To understand their behavior on a microscopic level, Holian et al. carried out Molecular Dynamics (MD) simulations [12, 13]. The MD simulations were able to yield macroscopic properties of shock waves, which matched Navier-Stokes continuum simulations and, additionally, to provide new insights in the relaxation process after the shock wave on an atomistic scale.

Experimentally, shock waves can be induced through a fast high energy deposition, which is used in the mass spectrometry (MS) technique Matrix Assisted Laser Desorption Ionisation(MALDI)-MS to isolate molecules into the gas phase. For this technique, aromatic compounds serve as solid matrices which absorb ultraviolet or infrared radiation from a laser. The radiation ionizes the matrix which transfers its charge to the analyt via ion-molecule reactions. In a second step, the heated matrix evaporates and transports the analyt into the gas phase. This desorption from the matrix and the ionization mechanism was investigated by MD-simulations [14,15,16,17,18,19], which assigned shock waves an important role in the desorption process. In a newly developed mass spectrometry technique called Laser Induced Liquid Beam Ion Desorption(LILBID)-MS, high energy is deposited on a liquid water filament, which serves as a matrix. The energy is deposited with an infrared laser tuned at the absorption wavelength of liquid water. The water filament is heated up and molecules as large as proteins can be transfered from the liquid into the gas phase without fragmentation [20,21]. In comparison to MALDI and other mass spectrometry techniques such as Electrospray Ionisation(ESI), this technique yields mostly singly charged 
molecules, which facilitates the identification of molecules in mixtures. In addition, the intensity obtained in the mass spectrum is proportional to the concentration of the molecule in the bulk, enabling a quantitative analysis. For biomolecules, using liquid water as matrix is a great advantage because they can be analyzed in their physiological environment.

However, little is known about the desorption process and the mechanism leading to mostly singly charged molecules.

The isolation of charges from different matrices represents a topic not only relevant for mass spectrometric techniques. In the atmosphere charges are separated in thunderstorms, thus, creating large electric fields in the order of $30 \mathrm{MV}$ between clouds and the earth's surface, which then provoke discharges of $20 \mathrm{kA}$ observed as lightnings. For this charge separation several mechanism were proposed. One possible effect may stem from large convection of air masses in the earlier stages of thunderstorms. Due to condensation at lower temperatures, the newly formed rain drops are polarized by the electrostatic field between the positive ionosphere and earth's negative surface and fall down. On their way down they separate negative ions to the bottom of the clouds and positive ions to the top (Wilson effect). Mason et al. [22] proposed another mechanism which relies on polarization of ice particles due to the electric field between the ionosphere and the earth. When rain drops hit these ice particles, they adopt their charge on the surface. These charged rain drops are separated in clouds and build the electric field resulting in the observed discharges.

Similar charging mechanism were also proposed for charged water clusters observed experimentally via mass spectrometry after the impact of neutral clusters on a surface [23]. Charge generation was assigned to the pickup of cations from the surface.

In order to study the charge mechanism responsible for the isolation of charged molecules from the liquid filament in the LILBID-technique, here, 
charge separation from liquid matrices is addressed with computer simulations. A general mechanism, which can be also applied to charge generation in the atmosphere, is worked out in Chapter 4. This mechanism is compared to measured data obtained via the LILBID-technique. Furthermore, the softness of the LILBID-technique is addressed in terms of transient temperature increase.

The following Chapter introduces the methods used to study the evolution of superheated metastable phases as well as the charge separation from liquid water. In the last Chapter, simulation results will be compared to the experiment and a general overview will be provided. 


\section{Chapter 2}

\section{Methods}

This Chapter describes different simulation methods used in this work to provide a molecular picture to experimental findings of fluids under extreme conditions. Thereby topics ranging from quantum mechanics to empirical force fields are addressed, which are used to reproduce properties of electrons and molecules in different experimental setups as a function of time. Also, tools for the analysis of the simulations in terms of e.g. hydrogen bonds which govern the structure in liquids like water and methanol up to supercritical conditions are discussed.

The properties of any physical system and its time evolution can be represented by equation 2.1, which is called the time-dependent Schrödinger equation.

$$
\hat{H}(t) \psi(t)=i \hbar \frac{\delta}{\delta t} \psi(t)
$$

$\hat{H}$ is the Hamiltonian describing the total energy of the system, $\psi(t)$ the wave function describing its state as a function of time, $i$ the imaginary unit, $\hbar$ the reduced Planck constant $\left(\frac{h}{2 \pi}\right)$.

For many systems the Hamiltonian is time independent and the time inde- 
pendent Schrödinger equation 2.2 can be used.

$$
\hat{H} \psi=E \psi
$$

By neglecting relativistic effects the time independent Hamiltonian can be written as the sum of a kinetic $\hat{T}$ and a potential energy operator $\hat{V}$. $E$ in equation 2.2 represents the total energy of the system.

As the aim of this thesis is to yield a molecular picture of fluids under extreme conditions, the respective wave functions has to be derived. The first step is the Born-Oppenheimer approximation, which allows treating the nuclear and electronic degrees of freedom separately.

\subsection{Born-Oppenheimer Approximation}

In the Born-Oppenheimer approximation electrons are treated as they would move in a field where the nuclei are frozen [24]. Since electrons are much lighter than nuclei, they move much faster and thus can adapt instantaneously to new configurations of nuclei. Within this approximation the time independent Schrödinder equation 2.2 for the electronic degrees of freedom for each configuration of the nuclei is solved.

The Ansatz consist in separating the wave function $\psi$ in a nuclear part $\psi_{n}$, which depends only on the nuclear positions and momenta and an electronic part $\psi_{e}$, which is a function of the electronic degrees of freedom, where the positions of the nuclei $\mathbf{R}_{n}$ enter as parameters.

$$
\psi=\psi_{n}\left(\mathbf{R}_{n}\right) \psi_{e}\left(\mathbf{r}_{e} ; \mathbf{R}_{n}\right)
$$

This wave function can be introduced into equation 2.2. If the influence of the nuclear kinetic energy operator on the electronic wave function $\psi_{e}$ is neglected, and the equation is multiplied with $\psi_{e}$ and integrated over the 
electronic degrees of freedom, the Schrödinger equation is given as

$$
\left(\hat{\mathbf{T}}_{n}+E_{e}\left(\mathbf{R}_{n}\right)\right) \psi_{n}\left(\mathbf{R}_{n}\right)=E_{t o t} \psi_{n}\left(\mathbf{R}_{n}\right)
$$

where $\mathbf{T}_{n}$ is the kinetic energy operator of the nuclei, $E_{e}$ the energy of the electronic Schrödinger equation $\hat{H}_{e} \psi_{e}\left(\mathbf{r}_{e}, \mathbf{R}_{n}\right)=E_{e} \psi_{e}\left(\mathbf{r}_{e} ; \mathbf{R}_{n}\right)$ for a given nuclei configuration and $E_{t o t}$ the total energy.

Solving the electronic Schrödinger equation for different nuclei configuration, which is described in detail in Chapter 2.4, yields the energy as function of nuclear positions. This forms the basis for the concept of the motion of nuclei on a Potential Energy Surface (PES).

\subsection{Classical Dynamics}

The Potential Energy Surface (PES) yields the change in energy of a system in respect to nuclear positions, but since in this work the dynamics of nuclei in fluids under extreme conditions is from interest, their motion on the PES has to be described. Here, the nuclei are treated classically with Newton's equation of motion (Classical Dynamics). Classical Dynamics is only valid for non bonded heavy atoms at high temperatures. Quantum mechanical effects of nuclear motions like tunneling of hydrogen atoms across potential barriers become more pronounced for small atoms at low temperatures. Under the assumption that quantum mechanical effects play a minor role in the studied systems, the positions of several atoms as a function of time can be derived from the underlying potential energy surface. How to achieve this using computer simulation represents the topic of the following Chapter. 


\subsection{Molecular Dynamics}

Via Molecular Dynamics(MD)-simulations the motion of atoms are followed under the assumption that the nuclei behave classically. The integration of Newton's equation of motion 2.5 for all atoms yields a trajectory for each atom in which its position as a function of time is described.

$$
\begin{aligned}
& \frac{d^{2} \mathbf{R}_{i}(t)}{d t^{2}}=\frac{\mathbf{F}_{i}}{m_{i}} \\
& \mathbf{F}_{i}=-\frac{\delta V\left(\mathbf{R}_{i}, \ldots, \mathbf{R}_{N}\right)}{\delta \mathbf{R}_{i}}
\end{aligned}
$$

The position of atom $i$ is denoted as $\mathbf{R}_{\mathbf{i}}$, the mass as $m_{i}, \mathbf{F}_{i}$ are the forces acting on this atom and $V\left(\mathbf{R}_{i}, \ldots, \mathbf{R}_{N}\right)$ is the underlying potential for this atom.

MD-simulations require the knowledge of the potential energy $V\left(\mathbf{R}_{i}, \ldots, \mathbf{R}_{N}\right)$ as a function of the coordinates of all atoms to calculate the force on each atom. This potential energy can be derived either from an empirical potential, which is discussed in Chapter 2.6, or calculated for each time step using quantum molecular mechanics, which is the topic of Chapter 2.4.

Once the potential energy as a function of the coordinates of all atoms is known, Newton's equation can be integrated for small time steps $\Delta t$. In order to keep the total energy constant throughout the integration $\Delta t$ has to be of the order of the fastest motion in the system of interest. For molecules, $\Delta t$ has to be smaller than the period of the fastest vibration (e.g. $\tau_{v i b}(\mathrm{OH})=10 \mathrm{fs}$ ) so that values of $1 \mathrm{fs}$ or $2 \mathrm{fs}$ are used. Constraints for the fastest motions enables larger time steps as they keep the constraint degrees of freedoms frozen throughout the simulation.

To integrate Newton's equation several algorithms have been proposed [25]. In this work the leap-frog algorithm is used, which uses the positions of the 
nuclei $\mathbf{R}$ at time $t$ and the velocities at time $t-\frac{\Delta t}{2}$.

$$
\begin{aligned}
\mathbf{R}(t+\Delta t) & =\mathbf{R}(t)+\mathbf{v}\left(t+\frac{\Delta t}{2}\right) \Delta t \\
\mathbf{v}\left(t+\frac{\Delta t}{2}\right) & =\mathbf{v}\left(t-\frac{\Delta t}{2}\right)+\frac{\mathbf{F}(t)}{m} \Delta t
\end{aligned}
$$

This algorithm is equivalent to the Verlet Algorithm [26], which is of the third order in $\mathbf{R}$ and time reversible.

\subsection{Quantum Mechanics}

Molecular Dynamics relies on the knowledge of the Potential Energy Surface and its derivative for a certain configuration of atoms. The timeindependent Schrödinger equation 2.2 and the Born-Oppenheimer approximation (Chapter 2.1) provide the basis for its calculation. The first step is to solve the electronic part of the Schrödinger equation for fixed positions of the nuclei. That means that the eigenfunctions to the electronic Hamilton operator $H_{e}$ have to be found, which is given in atomic units as

$$
\hat{H}_{e}=-\sum_{i}^{n_{e}} \frac{1}{2} \nabla_{e, i}^{2}-\sum_{i}^{n_{e}} \sum_{a}^{N} \frac{Z_{a}}{\mathbf{r}_{i, a}}+\sum_{i}^{n_{e}} \sum_{j \neq i}^{n_{e}} \frac{1}{\mathbf{r}_{i, j}} .
$$

$N$ is the total number of nuclei, $n_{e}$ the number of electrons and $Z_{a}$ is the atomic number of nuclei $a$. The first two terms depend on the position and momenta of only one electron. For one electron the last term cancels and the solution can be derived analytically. However, most molecules consist of more than one electron which require further approximations. The variational principle known from quantum mechanics plays a crucial role as it states that each trial wave function will yield a higher energy as the exact wave function (for a detailed argument see [27]). The energy of 
an arbitrary wave function $\psi$ is given by the following equation

$$
E=\frac{\langle\psi|\hat{H}| \psi\rangle}{\langle\psi \mid \psi\rangle} .
$$

The origin of the denominator arises from the normalization of the wave function, which ensures that the probability of finding the electrons anywhere is always one.

To find the wave function with the lowest energy some properties of electrons has to be considered. Electrons behave like fermions, which implies that the total wave function must be antisymmetric with respect to the interchange of coordinates of two electrons. This can be achieved by using a Slater Determinant (SD) for the total wave function in the form

$$
\psi=\frac{1}{\sqrt{N !}}\left|\begin{array}{cccc}
\phi_{1}(1) & \phi_{2}(1) & \cdots & \phi_{n}(1) \\
\phi_{1}(2) & \phi_{2}(2) & \cdots & \phi_{n}(2) \\
\cdot & \cdots & \cdots & \cdots \\
\phi_{1}(n) & \phi_{2}(n) & \cdots & \phi_{n}(n)
\end{array}\right|
$$

where $\mathrm{N}$ is the total number of electrons. The rows are formed by single electron wave functions (orbitals) $\phi_{n}$ depending on the spatial coordinates while the electron indices are along the rows.

Another property of electrons is their spin. In addition to the three cartesian coordinates describing the position of an electrons in space each electron has a property which is called spin. The spin is given by the operator $\hat{s}_{z}$ and takes values of $\pm \frac{1}{2} \hbar$. As the Hamiltonian is invariant to the spin a total description of the electron can be achieved by using spin orbitals which consist of a spatial and a spin part and can be written in vector notation as

$$
\varphi_{i}=\phi_{i}\left[\begin{array}{l}
\alpha \\
\beta
\end{array}\right] \text {. }
$$


If the total wave function is approximated by a single Slater Determinant formed out of orthonormal spin orbitals, the denominator for the energy in equation 2.10 vanishes and the energy is the sum of one and two electron integrals.

$$
E=\sum_{i}^{n}\left\langle\varphi_{i}\left|\hat{h}_{i}\right| \varphi_{i}\right\rangle+\sum_{i}^{n} \sum_{i \neq j}^{n}\left\langle\varphi_{i} \varphi_{j}\left|\frac{1}{\mathbf{r}_{i j}}\right| \varphi_{i} \varphi_{j}\right\rangle-\left\langle\varphi_{i} \varphi_{j}\left|\frac{1}{\mathbf{r}_{i j}}\right| \varphi_{j} \varphi_{i}\right\rangle
$$

Commonly instead of writing each integral a matrix notation as in equation 2.14 is used. $h_{i}$ is a matrix element representing the one electron integral which describes the kinetic energy and the potential energy arising from the electron-nucleus interaction of an electron in the spin orbital $\varphi_{i}$.

$$
E=\sum_{i}^{n} h_{i}+\sum_{i}^{n} \sum_{j \neq i}^{n} J_{i j}-K_{i j}
$$

$J_{i j}$ is called the Coulomb integral and equals $\left\langle\varphi_{i} \varphi_{j}\left|\frac{1}{\mathbf{r}_{i j}}\right| \varphi_{i} \varphi_{j}\right\rangle$. This integral accounts for the electrostatic interactions of two charge distributions $\left|\varphi_{i}\right|^{2}$ and $\left|\varphi_{j}\right|^{2}$. The second integral $K_{i j}$ is called the exchange integral and equals $\left\langle\varphi_{i} \varphi_{j}\left|\frac{1}{\mathbf{r}_{i j}}\right| \varphi_{j} \varphi_{i}\right\rangle$. This integral has no classical analog and is the result of the antisymmetry of the wave function.

Although this forms the basis for the calculation of the energy of a single Slater Determinant representing the total wave function, still an algorithm is needed which yields the wave function with the lowest energy. One of the first algorithm is the Hartree-Fock method. 


\subsubsection{Hartree-Fock Method}

The Hartree-Fock method allows an iterative search for the wave function with the lowest energy. The best orbitals has to be found, which minimize the energy in respect to little changes in the orbitals denoted as $\delta \varphi_{i}$, under the condition that the orbitals remain orthonormal. The resulting minimized orbitals are given by the Hartree-Fock equation (for a detailed description see [27]).

$$
\hat{F}_{i} \tilde{\varphi}_{i}=\varepsilon_{i} \tilde{\varphi}_{i}
$$

The Fock operator $\hat{F}_{i}$ is defined as

$$
\hat{F}_{i}=\hat{h}_{i}+\sum_{j} \hat{J}_{j}-\hat{K}_{j}
$$

$\hat{J}_{j}$ and $\hat{K}_{j}$ are two electron operators of the form $\hat{J}_{j} \varphi_{i}=\left\langle\varphi_{j}\left|\frac{1}{\mathbf{r}_{i j}}\right| \varphi_{j}\right\rangle \varphi_{i}$ and $\hat{K}_{j} \varphi_{i}=\left\langle\varphi_{j}\left|\frac{1}{\mathbf{r}_{i j}}\right| \varphi_{i}\right\rangle \varphi_{j}$ and $\hat{h}_{i}$ is an one electron operator describing the kinetic energy contribution and the electrostatic interaction with all nuclei of the electron $i . \tilde{\varphi}_{i}$ are the orbitals which diagonalize the Fock matrix and $\varepsilon_{i}$ represent the energy of each orbital.

The operator $\hat{J}_{j}$ is called the Coulomb-operator and describes the average electron potential felt by an electron in orbital $i$ (see 2.17). The instantaneous position of the other electron contributing to the total electron potential acting on electron in orbital $i$ is replaced by an average interaction over all spin and spatial coordinates of the other electrons. The operator $\hat{J}_{j}$ is called a local operator, since it depends only on the position of the electron in orbital $i$. 


$$
v_{\text {coul }}(i)=\sum_{j \neq i}\left\langle\varphi_{j}\left|\frac{1}{\mathbf{r}_{i j}}\right| \varphi_{j}\right\rangle
$$

The operator $\hat{K}_{j}$ is called the exchange operator, since it exchanges an electron from orbital $i$ to $j$. This operator is in contrast to the coulomboperator a nonlocal operator as it does not only depend on the electron in orbital $i$ but also in orbital $j$.

Because the spatial parts of the molecular spin orbitals are unknown, the Linear Combination of atomic Orbitals (LCAO) method is used. This method describes a molecular orbital as a sum of atomic orbitals multiplied with certain coefficients $c_{k i}$. For computational reasons usually Gauss functions $\chi_{k}$ are used.

$$
\phi_{i}=\sum_{k} c_{k i} \chi_{k}
$$

Replacing the molecular orbitals in equation 2.15, multiplying from the left with $\phi_{j}^{*}$ and integrating yields the Roothaan-Haal equations.

$$
\sum_{k} \mathbf{F}_{j k} \mathbf{C}_{k i}=\varepsilon_{i} \sum_{k} \mathbf{S}_{j k} \mathbf{C}_{k i}
$$

$\mathbf{F}_{j k}$ is the Fock matrix with elements $\int \chi_{j}^{*} \hat{F}(1) \chi_{k} d r_{1}$ and the dimension $K \times K$ ( $K$ being the number of Gauss-functions). $\mathbf{S}_{j k}$ is called the overlap matrix, which diagonal elements are one and the off-diagonal elements have absolute values less than one, since the used Gauss functions are normalized but not orthogonal to each other. The set of equations can be rewritten in Matrix-form (2.20), where $\mathbf{C}$ is a $K \times K$ matrix with elements $c_{k i}$ and $\varepsilon$ is 
a diagonal matrix with the diagonal values $\varepsilon_{i}$.

$$
\mathbf{F C}=\mathbf{S C} \varepsilon
$$

Since the Fock-matrix is also dependent on the coefficients $c_{k i}$, the coefficients have to be optimized iteratively in a self consistent field approach. The advantage is that the integrals have to be evaluated only once.

\subsubsection{Koopmans' Theorem}

One of the aims of this thesis is to understand changes in the energy of electrons measured via photoelectron spectra during an evolution of a metastable liquid. These energies equal the vertical ionization potential of an electron in a specific orbital, if the ionization process is carried out without changes in the position of the nuclei. This vertical ionization potential is obtained via the total energy difference of the neutral and the ionized molecule at the same configurations, where an electron is removed from the specific orbital. Koopmans' theorem neglects any changes of the orbitals during this process ("frozen $M O$ "-approximation). With this approximation the diagonal elements of the diagonalized Fock-matrix in the Hartree-Fock method, which represent the energies of the canonical molecular orbitals, are used to calculate vertical ionization potentials.

The ionization potential is obtained from the energy difference of two Slater determinants. One with $N$-occupied orbitals and one with $(N-1)$-occupied orbitals, where one electron has been removed from orbital $k$. The respec- 
tive energies are given as

$$
\begin{array}{r}
E_{N}=\sum_{i}^{N} h_{i}+\sum_{i}^{N} \sum_{j \neq i}^{N} J_{i j}-K_{i j} \\
E_{N-1}^{k}=\sum_{i}^{N-1} h_{i}+\sum_{i}^{N-1} \sum_{j \neq i}^{N-1} J_{i j}-K_{i j} .
\end{array}
$$

The energy difference between equation 2.21 and 2.22, which corresponds to the ionization potential within the "frozen $M O$ "-approximation for removing an electron from orbital $k$, is the orbital energy of orbital $k$.

$$
E_{N}-E_{N-1}^{k}=h_{k}+\sum_{i}^{N} J_{k i}-K_{k i}=\varepsilon_{k}
$$

\subsubsection{Electron Correlation}

The Hartree-Fock approximation (2.4.1) is a good first approximation for finding the wave function with the lowest energy for a molecule at a certain conformation. But as the interaction of the electrons is only treated in a mean field approach (eq. 2.17) no correlation between the electrons is present. The difference between the Hartree Fock energy and the total energy is called the electron correlation energy, which is a consequence of the motion of the electrons being correlated. This energy represents only $\sim 1 \%$ of the total energy. But since total energies are very large, the electron correlation makes an important contribution in quantum chemistry (especially in hydrogen bonded systems) where small energy differences are of interest.

Nevertheless, the Hartree-Fock method serves as the first step going to more advanced methods - i.e. Configuration Interaction (CI) or Coupled Cluster(CCSD,CCSD (T) ) - where electron correlation is taken into account. 
For a nice introduction and overview the reader is referred to [27].

Here, a short introduction into perturbation theory is given, as this is the method used for accounting for the electron correlation of hydrogen bonded liquids in this work.

In perturbation theory one is interested in knowing the change in energy of a system by applying a small perturbation. The Hamilton operator is represented as the sum of the Hamilton operator of the unperturbed system and the perturbation operators of different orders.

$$
\hat{H}=\hat{H}_{0}+\lambda \hat{H}_{1}+\lambda^{2} \hat{H}_{2}+\ldots+\lambda^{n} \hat{H}_{n}
$$

Since the perturbation is expected to be small compared to the unperturbed system the energy and the wave function can be expanded in a Taylorseries.

$$
\begin{gathered}
E=E_{0}+\lambda E_{1}+\lambda^{2} E_{2}+\ldots \lambda^{n} E_{n} \\
\psi=\psi_{0}+\lambda \psi_{1}+\lambda^{2} \psi_{2}+\ldots \lambda^{n} \psi_{n}
\end{gathered}
$$

Replacing the terms into the Schrödinger equation yields

$$
\sum_{m} \lambda^{m} \sum_{n}\left[\hat{H}_{k}-E_{k}\right] \psi_{m-n}=0 .
$$

The sum can only be zero, if all the coefficients are zero. The equation can be written for each contribution of the Taylor expansion until the respective order to the total energy. In the following only the contribution until the second order are considered.

$$
\begin{array}{ll}
m=0: & \hat{H}_{0}-E_{0} \psi_{0}=0 \\
m=1: & \hat{H}_{0}-E_{0} \psi_{1}+\hat{H}_{1}-E_{1} \psi_{0}=0 \\
m=2: & \hat{H}_{0}-E_{0} \psi_{2}+\hat{H}_{1}-E_{1} \psi_{1}+\hat{H}_{2}-E_{2} \psi_{0}=0
\end{array}
$$


In the Rayleigh-Schröder perturbation theory the intermediate normalization is chosen such that the wave functions $\psi_{0}, \psi_{k}$ are orthogonal. By the multiplication of equations 2.29 and 2.30 with $\psi_{0}$ from the left the respective energies are given as

$$
\begin{aligned}
& E_{0}=\left\langle\psi_{0}\left|\hat{H}_{0}\right| \psi_{0}\right\rangle \\
& E_{1}=\left\langle\psi_{0}\left|\hat{H}_{1}\right| \psi_{0}\right\rangle \\
& E_{2}=\left\langle\psi_{0}\left|\hat{H}_{1}\right| \psi_{1}\right\rangle+\left\langle\psi_{0}\left|\hat{H}_{2}\right| \psi_{1}\right\rangle
\end{aligned}
$$

As can be seen in equation 2.33 to get the energy of $(n+1)$-order only the wave function of the $n$-order must be known. If $\hat{H}_{2}$ is zero the second order energy can be solved, if one knows the wave function to the first order. To get a solution for the first order wave function, equation 2.29 can be used and the first order wave function is expanded as linear combination of the known eigenfunctions of $\hat{H}_{0}$. After finding the coefficients, which minimize the energy, the second-order energy can be written as

$$
E_{2}=\sum_{i} \frac{\left\langle\psi_{0}\left|\hat{H}_{1}\right| \psi_{i}\right\rangle\left\langle\psi_{i}\left|\hat{H}_{1}\right| \psi_{0}\right\rangle}{E_{0}-E_{i}}
$$

In quantum chemistry the Rayleigh-Schröder intermediate normalization is used in the Møller-Plesset method to calculate the contribution of the electron correlation to the total energy. The unperturbed operator $\hat{H}_{0}$ is a sum of Fock operators and the perturbation operator is given in equation 2.36.

$$
\begin{aligned}
& \hat{H}_{0}=\sum_{n} \hat{F}_{i} \\
& \hat{H}_{1}=\hat{J}_{j}-\hat{K}_{j}+2 \sum_{n} \sum_{j \neq i} \hat{J}_{i j}-\hat{K}_{j i}
\end{aligned}
$$

Using this approach the zero-order energy is the sum of the orbital energy and the first-order energy is the Hartree-Fock energy. The second-order 
energy is given as

$$
E_{2}=\sum_{i} \sum_{j \neq i} \sum_{a} \sum_{b \neq a} \frac{\left\langle\psi_{0}\left|\hat{H}_{1}\right| \psi_{i j}^{a b}\right\rangle\left\langle\psi_{i j}^{a b}\left|\hat{H}_{1}\right| \psi_{0}\right\rangle}{E_{0}-E_{i j}^{a b}}
$$

where $\psi_{i j}^{a b}$ denotes the excitation of two electrons from orbital $i$ and $j$ into $a$ and $b$. Single excitations don't contribute to the second-order energy because of Brillouins theorem (see [27]), which states that the expectation value of the Hamilton operator with $\psi_{0}$ and $\psi_{i}^{a}$ is zero because of the orthogonality of the zero-order and the first-order wave function.

Compared to other methods accounting for electron correlation MP2 is computationally faster and yields good results when treating hydrogen bonded systems. Other methods may recover more from the electron correlation energy but are computationally very demanding. As will be seen in this work a compromise between accuracy in the energy and sampling efficiency (maximal amount of calculated configurations per state) is needed to reproduce experimental observables.

\subsection{Quantum Mechanics Molecular Mechanics Method}

Quantum mechanical methods, such as Hartree Fock or higher level methods like Møller Plesset are able to give an accurate description of the potential energy surface. But since this methods are computationally very expensive only systems in the order of 100 atoms can be treated. Semi-empirical methods account for larger systems at a lower level of accuracy, but the system sizes reached are still not able to reproduce long range effects which are important in studying influences of solvents on electron energies obtained from photoelectron spectra. 


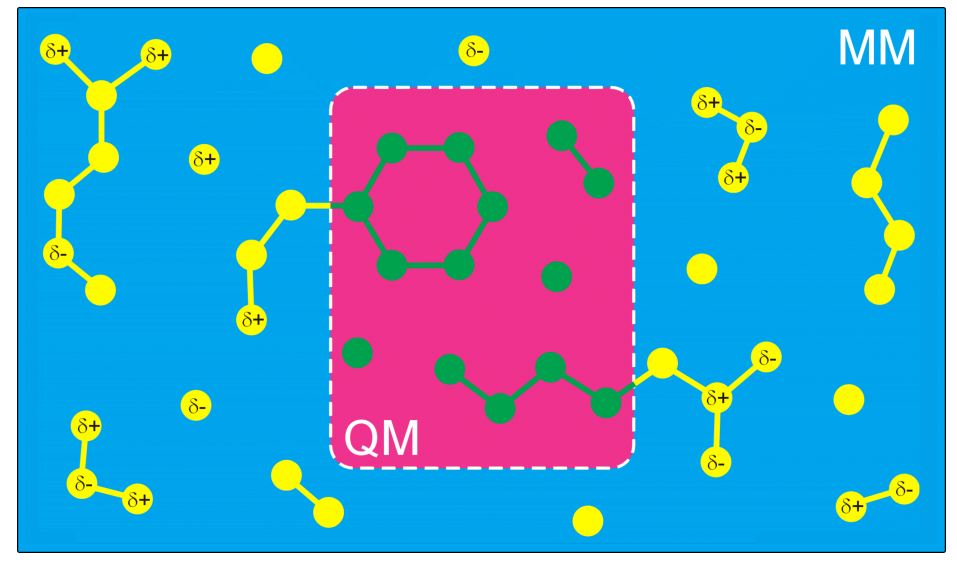

Figure 2.1: Scheme for the QM/MM-embedding.

One way to increase the system size and thereby include long range effects of the environment when performing simulations in solution or in biological systems is given by Hybrid Quantum mechanical / Molecular Mechanics (QM/MM) methods in which the environment is treated with an empirical force field $[28,29,30,31]$. A schematic picture is shown in Fig 2.1. This method proposed by Warshel and Levitt [32] uses the effective Hamiltonian in the form

$$
\hat{H}_{\mathrm{eff}}=\hat{H}_{\mathrm{QM}}+\hat{H}_{\mathrm{MM}}+\hat{H}_{\mathrm{QMMM}}^{\mathrm{elec}}+\hat{H}_{\mathrm{QMMM}}^{\mathrm{vdW}}
$$

$\hat{H}_{\mathrm{QM}}$ in equation 2.38 is the Fock-operator of equation 2.15 if the HartreeFock method was used (but can be replaced by electronic Hamiltonians of other approximations, i.e. MP2, semiempirical methods), plus the electrostatic interactions between the QM nuclei. The $\hat{H}_{\mathrm{MM}}$ operator represents the Hamilton operator which is used in empirical force fields and consists of Coulomb, Van der Waals, and bonded interactions and will be explained in detail in the following Chapter.

When no electrostatic interactions between the quantum mechanical- (QM) and the molecular mechanical- (MM) part are present, only the $\hat{H}_{\mathrm{QMMM}}^{\mathrm{vdW}}$ 
operator remains. This method is called mechanical embedding. The two parts interact only via Van der Waals interaction described at the MM level. This interactions ensure that the MM-part and the QM-part do not come too close due to the absence of electronic structural description of the MM atoms.

$$
\hat{H}_{\mathrm{QMMM}}^{\mathrm{vdW}}=\sum_{k}^{N_{\mathrm{QM}}} \sum_{l}^{N_{\mathrm{MM}}} 4 \varepsilon\left[\left(\frac{\sigma_{k l}}{\mathbf{R}_{k l}}\right)^{12}-\left(\frac{\sigma_{k l}}{\mathbf{R}_{k l}}\right)^{6}\right]
$$

$N_{\mathrm{QM}}$ is the number of nuclei in the QM- and $N_{\mathrm{MM}}$ the number in the MM-region. The parameters have to be optimized to describe properly the non-bonding interaction between the two systems.

Normally the electrostatic contribution of the environment has also to be taken into account since it induces a polarization of the wave function in the QM-part of the system. This method is called electronic embedding and the operator $\hat{H}_{\mathrm{QMMM}}^{\text {elec }}$ forms part of the effective Hamiltonian (2.38).

$$
\hat{H}_{\mathrm{QMMM}}^{\text {elec }}=-\sum_{i}^{n_{e}} \sum_{l}^{N_{\mathrm{MM}}} \frac{Q_{l}}{\mathbf{r}_{i, l}}+\sum_{a}^{N_{\mathrm{QM}}} \sum_{l}^{N_{\mathrm{MM}}} \frac{Z_{a} Q_{l}}{\mathbf{R}_{a, l}}
$$

$n_{e}$ represents the total number of electrons, $Q_{l}$ is the charge of MM atom $l$ and $Z_{a}$ is the charge of the QM nuclei $a$. Adding this operator to the Fock-operator yields a new one-electron operator (2.41), which describes the interaction of each electron in his molecular orbital with the charges of the MM atoms and leads to the polarization of the wave function.

$$
\hat{h}_{i}=-\frac{1}{2} \nabla_{e, i}^{2}-\sum_{a}^{N_{\mathrm{QM}}} \frac{Z_{a}}{\mathbf{r}_{i, a}}-\sum_{l}^{N_{\mathrm{MM}}} \frac{Q_{l}}{\mathbf{r}_{i, l}}
$$

The disadvantage of the electronic embedding method consist in not accounting for the polarization of the MM-part caused by the QM-part. Methods which take the polarization of the MM-region into account are 
called polarizable embedding methods, but require a polarizable force field for the MM-part of the system.

The aim of this work was to study the influence of a water environment on the orbital energies of water clusters and therefore the electronic embedding method, as implemented by Groenhof et al. [33] in the GROMACS-package and GAUSSIAN, is used.

\subsection{Empirical Force Fields}

Since the number of atoms which can be treated by Quantum Mechanics is limited to 100 atoms one has to fall back to empirical methods when dynamical properties of solvents are of interest. This methods allow to treat systems in the size of $>10^{6}$ atoms.

In empirical methods the interactions between atoms are described with a potential $V$ which consists of non-bonded and bonded terms.

$$
V=\sum_{\text {non-bond }} V_{\text {non-bond }}+\sum_{\text {bond }} V_{\text {bond }}
$$

One of the non-bonded terms is the Coulomb term, which describes the interaction between two charged atoms of charge $Q_{i}$ (equation 2.43 first term). The Van der Waals attractive interaction between two atoms, originated from induced dipoles produced by electron fluctuation in the electron cloud of an atom and the Pauli repulsion, which describes the repulsion of the electron clouds of two atoms at short distances make the second contribution to the non-bonded interaction and are represented by the Lennard- 
Jones potential.

$$
\sum_{\text {non-bond }} V_{\text {non-bond }}=\sum_{i}^{N} \sum_{j \neq i}^{N}\left[\frac{Q_{i} Q_{j}}{4 \pi \epsilon_{0} \mathbf{r}_{i j}}+\left(\frac{\sigma_{12, i j}}{\mathbf{r}_{i j}}\right)^{12}-\left(\frac{\sigma_{6, i j}}{\mathbf{r}_{i j}}\right)^{6}\right]
$$

$\sigma_{6, i j}$ and $\sigma_{12, i j}$ are the Lennard-Jones parameters for atoms $i$ and $j$ and $\mathbf{r}_{i j}$ the distance. The atomic charges and the Lennard-Jones parameters must be parameterized by comparing observables obtained from simulations with their experimental analog.

The bonded terms in the potential describe covalent interactions between two atoms, bond angles or dihedral interactions.

$$
\begin{aligned}
\sum_{\text {bond }} V_{\text {bond }}= & \sum_{i j}^{\text {bonds }} \frac{1}{2} K_{b, i j}\left(r_{e, i j}-r_{i j}\right)^{2}+ \\
& \sum_{i j k}^{\text {angles }} \frac{1}{2} K_{a, i j k}\left(\theta_{e, i j k}-\theta_{i j k}\right)^{2}+ \\
& \sum_{i j k l}^{\text {torsions }} \frac{1}{2} K_{\text {tor }, i j k l}\left(1+\cos \left(n_{i j k l} \phi_{i j k l}-\phi_{i j k l}^{0}\right)\right)^{2}
\end{aligned}
$$

As in the case for the non-bonded interactions parameters like the force constants $K_{i j}$ and the equilibrium distances $r_{e, i j}$ and angles $\theta_{e, i j k}$ must be parameterized e.g. by Quantum Mechanical calculations.

A given set of parameters for the non-bonded and bonded interactions are bundled in a force field, which describe the interactions for a set of molecules and atoms. Popular force fields are GROMOS [34], OPLS [35], CHARMM [36] and AMBER [37]. In this work the GROMOS and OPLS force fields are used as they provide good parameters for the simulation of water and methanol. 


\subsubsection{Water Models}

The reliability of force fields depends crucially on the parameters which are used. In the case of water several models with different parameters and monomer geometries have been proposed in literature and still form part of ongoing research [38]. A complete review of the several models, their advantages and disadvantages can be found in [39].

The first water model was proposed by Bernal and Fowler [40] in 1933 and consisted of a tetrahedral distribution of the charges around the oxygen atom two of them sitting on the hydrogen atoms. Almost 40 years later in 1974 Stillinger and Rahman [41] proposed also a water model with four point charges (Fig. 2.2a) but a different geometry: two positive charges of $+0.24 \mathrm{e}$ on the hydrogens at a distance of $1 \AA$ from the oxygen and two negative ones of -0.24 e at $0.82 \AA$ give a tetrahedral conformation. A Lennard-Jones potential on the oxygen takes the short range repulsion into account. The less symmetrical charge distribution compared to the model of Bernal and Fowler led to less tetrahedral structure observed in liquid water.

One of the most popular water models used in computer simulations

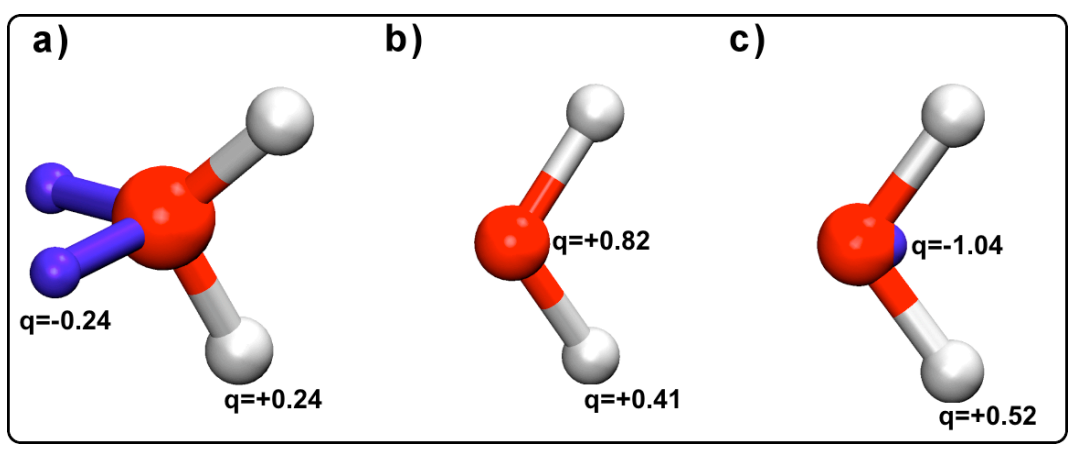

Figure 2.2: Water models a)Stillinger and Rahman (red oxygen, white hydrogens, blue extra charges) b) Berendsen (charges only on oxygen and hydrogens) c) TIP4P (charges on hydrogens and negative charge on bisector).

nowadays are the simple point charge (SPC) model of Berendsen [42] and 
Table 2.1: Thermodynamic properties of the SPC- and the TIP4P model

\begin{tabular}{|l|c|c|c|}
\hline & SPC & TIP4P & Experiment \\
\hline$H_{\text {vap }}(\mathrm{kJ} / \mathrm{mol})$ & 45.06 & 44.60 & 43.97 \\
\hline$\mu(\mathrm{D})$ & 2.3 & 2.2 & 3.0 \\
\hline$\epsilon_{R}$ & 63 & 60 & 78 \\
\hline$D\left(10^{5} \mathrm{~cm}^{2} / \mathrm{s}\right)$ & 3.85 & 3.29 & 2.30 \\
\hline$\kappa\left(10^{6} \mathrm{bar}^{-1}\right)$ & 27 & 35 & 46 \\
\hline$T_{\text {crit }}(\mathrm{K})$ & 575 & 560 & 642 \\
\hline$\rho_{\text {crit }}\left(\mathrm{g} / \mathrm{dm}^{3}\right)$ & 260 & 290 & 323 \\
\hline
\end{tabular}

the transferabel intermolecular potential four points (TIP4P) model of Jorgensen [43] (see Fig. 2.2b and 2.2c).

The SPC model has a charge of $-0.82 \mathrm{e}$ on the oxygen and $+0.41 \mathrm{e}$ on the hydrogens yielding a total dipole moment of $2.5 \mathrm{D}$. The $\mathrm{OH}$-distance is $1 \AA$ and the OHO-angle $109.4^{\circ}$. The TIP4P model has a slightly smaller $\mathrm{OH}-$ distance of $0.9572 \AA$ and an OHO-angle of $104.5^{\circ}$ (see Tab. ??). Also the charge distribution differs from the one in the SPC model. The charge on the oxygen is replaced by a charge of $-1.04 \mathrm{e}$ on the bisector between the two OH-bonds at a distance of $0.15 \AA$ from the oxygen atom and the two charges on the hydrogens constitute $+0.52 \mathrm{e}$. This charge distribution leads to a slightly smaller dipole moment of $2.2 \mathrm{D}$.

Apart from the two mentioned models several models can be found in literature reproducing different thermodynamic properties of water with varying accuracy. The TIP5P model [44] for example is able to reproduce the density dependence of liquid water on temperature very well but suffers in the description of the self diffusion and has a higher computational cost due to five interaction sites. Polarizable models like the TIP4P/FQ [45] model did not succeed in improving the overall thermodynamic properties. The SPC/E model of Berendsen [46] which accounts for the polarizability of a molecule in an average way is known to reproduce the phase diagram of 


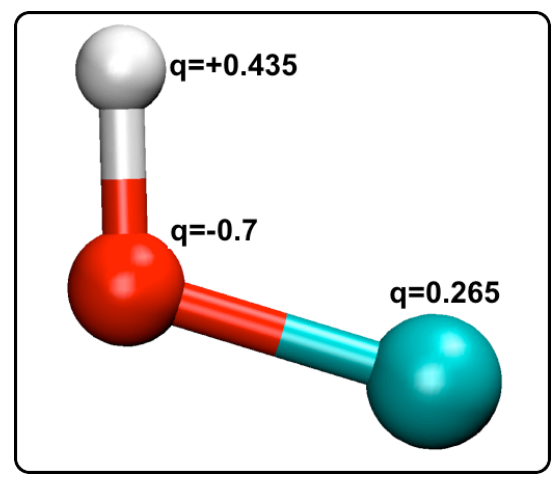

Figure 2.3: OPLS methanol model (white hydrogen atom, red oxygen atom, green $\mathrm{CH}_{3}$-group).

water very well at a computational low cost.

In this work the SPC and the TIP4P model are used since they are able to reproduce most of the thermodynamic properties of water in a large temperature and pressure range at a low computational cost. In several cases the influence of the model on the results is verified by performing the same simulations with different models. For the studied systems no large influence on the results could be seen. As an overview, Table 2.1 shows the most relevant thermodynamic properties of the SPC and TIP4P model in comparison to experimental values [39].

\subsubsection{Methanol Models}

One of the most complete studies of force field parameters for liquid alcohols was performed by Jorgensen with the transferable interatomic potential functions (TIPS) $[47,43,48]$ and introduced in the Optimal Parameters for Liquid Simulation (OPLS) force field [35].

The model describes the methanol monomer as a three sites particle represented by a carbon-, a hydrogen atom and a methyl group (see Fig. 2.3). Bond lengths and angles are taken from experimental microwave experi- 
Table 2.2: Geometrical properties of OPLS methanol monomer

\begin{tabular}{|l|l|}
\hline O-H-length $(\AA)$ & 0.945 \\
\hline C-O-length $(\AA)$ & 1.430 \\
\hline COH-angle $\left(^{\circ}\right)$ & 108.5 \\
\hline
\end{tabular}

ments (Table 2.2) and the charges on the atoms are distributed to yield a $25 \%$ larger dipole moment than the gas phase value (2.2 D). Lennard-Jones parameters for the oxygen and the methyl group are first assigned to yield the correct interaction energies of the methanol dimer and methanol water dimer (TIP4P). The obtained parameters are then refined to match the experimental density and internal energy of the liquid.

Although this model is parameterized for liquid methanol at normal conditions, it was also applied for the simulations of methanol at supercritical conditions and compared to experimental pulsed neutron diffraction data $[49,50]$. It reproduced thermodynamic properties of supercritical methanol very well (Table 2.3) and to matched the experimental average number of hydrogen bonds per molecule from NMR studies.

The thermodynamic properties are summed up in Table 2.3.

Table 2.3: Thermodynamic properties of OPLS methanol model

\begin{tabular}{|l|c|c|}
\hline & OPLS & Experiment \\
\hline$\rho_{298 K, 1 \text { bar }}\left(\mathrm{g} / \mathrm{dm}^{3}\right)$ & 759 & 789 \\
\hline$H_{\text {vap }}(\mathrm{kJ} / \mathrm{mol})$ & 37.83 & 37.37 \\
\hline$T_{\text {crit }}(\mathrm{K})$ & 493 & 512 \\
\hline$\rho_{\text {crit }}\left(\mathrm{g} / \mathrm{cm}^{3}\right)$ & 250 & 279 \\
\hline
\end{tabular}




\subsection{Hydrogen Bonds}

Hydrogen bonds play an important role in fluids like water and methanol. They provide an attraction between an acceptor atom with a lone electron pair (e.g. oxygen, nitrogen, fluor) and a donor atom with a hydrogen atom (e.g. $\mathrm{OH}, \mathrm{NH}$ ). Depending on the system the binding energies may range from 5 to $60 \mathrm{~kJ} / \mathrm{mol}$.

The definition of hydrogen bonds can vary in literature. In this thesis a geometric definition is used. A donor acceptor pair is defined as hydrogen bonded when the oxygen-oxygen distance is smaller than $3.5 \AA$ and the angle between donor-hydrogen- and acceptor-hydrogen vector is smaller than $30^{\circ}$.

The binding energy of a hydrogen bond can be calculated for small systems with quantum chemical calculations. It is given as the energy difference between the energy of the hydrogen bonded complex and the energies from acceptor and donor. But if the analysis has to be performed for systems with several atoms and over an ensemble of configurations quantum chemical calculations become prohibitive. Therefore, in this work the empirical relation between binding energy and donor-hydrogen distance from Espinosa et al. [51] is used.

$$
E_{\mathrm{Hb}}=2.5 \cdot 10^{4} \exp (-36 \cdot d)
$$

$d$ represents the distance between hydrogen- and acceptor atom in $\AA$. This formula is based on experimental electron densities obtained from small different hydrogen bonded systems measured with X-ray and Neutron scattering techniques. Together with quantum chemical calculation of the structures from small hydrogen bonded systems an exponential relation between distance and binding energy could be obtained. In this work this formula should reveal changes in the hydrogen bond energetics during the simulation and is not intended to give exact absolute values. 


\subsubsection{Hydrogen Bond Kinetics}

The formation and dissociation of hydrogen bonds in liquids like water and alcohols play a crucial role in their structure and dynamics. Although there are some experimental techniques which can probe the kinetics indirectly, computer simulations can give a microscopic picture of the dynamics of hydrogen bonds and thus enable the calculation of their lifetimes [52].

As noted in Chapter 2.7 several definitions for a hydrogen bond can be found in literature. Also the procedures to obtain their lifetimes vary throughout the literature of the last decades [53]. Thereby, two main approaches using autocorrelation function for molecular pairs $C(t)$ have been proposed: The first evaluates the autocorrelation function for continuously bonded hydrogen bonds, i.e. which did not suffer a breaking in the interval from 0 to $t$. The second approach uses the autocorrelation function of uninterrupted hydrogen bonds, i.e. not accounting for intervening interruptions $[54,53,55]$. Under the assumption that the probability of formation and dissociation of a hydrogen bond is constant and independent of the hydrogen bond history the autocorrelation function should fall exponentially. The overall hydrogen bond lifetime is given as

$$
\tau_{\mathrm{HB}}=\int_{0}^{\infty} C(t) d t
$$

With the continuum definition, the autocorrelation function $C(t)$ depends on the number of configurations which are analyzed during the time interval $\Delta t$. The reason is that if the number of configuration in $\Delta t$ increases more interruptions are observed and the correlation function will fall off faster and the overall lifetime gets smaller. In MD-simulation the number of configurations in $\Delta t$ is given by the frequency in which the coordinates of the system are saved. The limiting factor for the saving frequency is the time step used to integrate Newton's equations, which represents the minimal interval between two configurations and makes this approach not 
feasible.

But the definition of the uninterrupted hydrogen bonds does not suffer from the saving frequency, since it neglects all events in the period between 0 and $t$. With this definition a binary function for each hydrogen bond $h(t)$ can be defined, which is 1 if the hydrogen bond is formed at time $t$ and otherwise 0 . From the autocorrelation function of these function averaged for all hydrogen bonds $\left(C_{h}(t)\right)$ the overall lifetime for a hydrogen bond can be obtained. Given that the simulation systems are finite $C_{h}(t)$ does not reach zero. For this cases $C_{h}(t)$ is scaled to zero at long times.

In this thesis the analysis tool implemented in the GROMACS-package with version 3.3.1 based on the above description is used for the lifetimes of hydrogen bonds in liquid water and methanol at normal and supercritical conditions. 



\section{Chapter 3}

\section{Ultrafast Dynamics of Metastable Liquids}

Water, in contrast to other hydrogen bonded liquids like methanol, reveals ultrafast dynamics, when heated up to above their critical point in vacuum. This Chapter describes first the experimental setup and the method used to study the ultrafast dynamics of water and methanol. Computer simulation will provide the underlying microscopic picture and will explain the different behavior observed for these two liquids.

\subsection{Experiment}

The experiments to study the dynamics of metastable liquids are described in detail in [11]. Here only a short overview will be presented.

To transfer liquids into a metastable state an ultrafast femtosecond near infrared laser $(\lambda=2.6-3.0 \mu \mathrm{m})$ tuned at the $\mathrm{OH}$-stretch vibration of water (methanol) is used, which leads to an instantaneous heating of the sample. The fast energy deposition does not allow for an expansion of the liquid, such that a superheated metastable phase is created. The achieved 


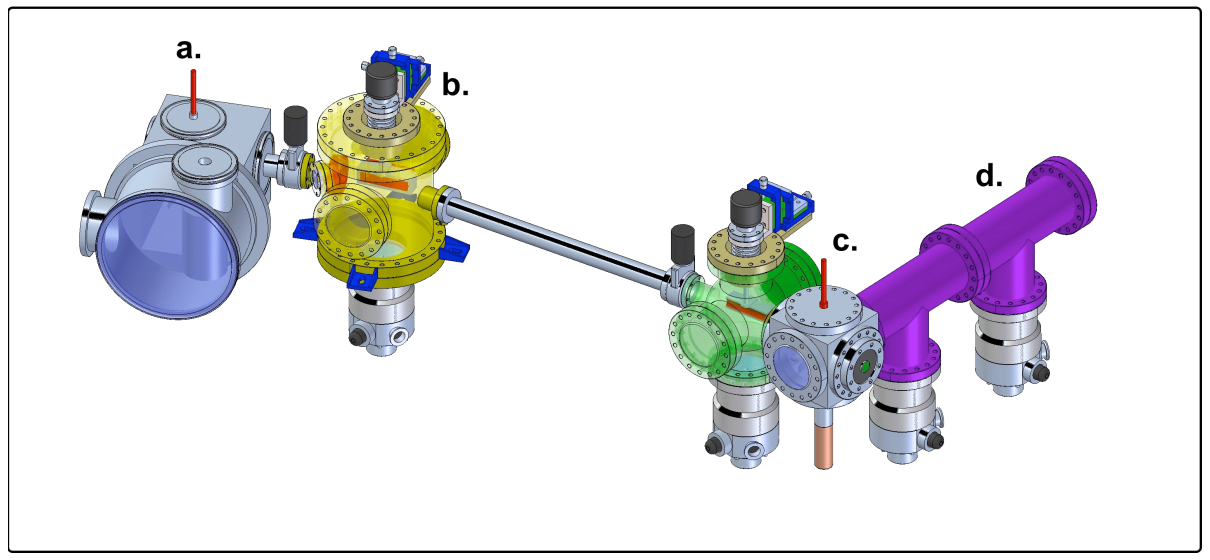

Figure 3.1: Experimental setup: a.) Capillary flown with argon for High Harmonic Generation, b.) Grating for selection of the High Harmonics, c.) Liquid beam in vacuum chamber, d.) Time of flight detector for photoelectrons.

internal energies are in the order of 10 to $45 \mathrm{~kJ} / \mathrm{mol}$ corresponding to temperatures from 450 to $1100 \mathrm{~K}$. The subsequent dynamics are traced with EUV-radiation generated by High Harmonic generation from an argon capillary. To this end, the 32 -th harmonic $(40 \mathrm{eV})$ is selected with a grating and focused on the sample with a toroidal mirror. The high energy radiation generates photoelectrons whose kinetic energy is analyzed with a time of flight spectrometer (Fig. 3.1).

Because of the large cross section of the emitted photoelectrons the whole experimental setup has to be kept in a vacuum chamber with a pressure smaller than $10^{-5}$ mbar. To combine this required low pressure and the volatility of the liquids the technique of Faubel et al. $[56,57]$ is used. A liquid microjet of $10 \mu \mathrm{m}$ diameter is generated by pumping $0.2-0.4 \mathrm{ml} / \mathrm{min}$ of liquid trough a nozzle of the same diameter (Fig. 3.2a). Because of the large velocity with which new liquid is provided $(10-50 \mathrm{~m} / \mathrm{s})$, a freezing out of the jet can be avoided. Via this technique a vacuum-liquid interface is created in which the liquid is under normal atmospheric conditions. Some millimeters after the liquid leaves the nozzle it becomes unstable and droplets are 
formed (Fig. 3.2b).

The recorded electrons in the photoelectron spectra are ejected from molecules in the first nanometer of the liquid-vacuum interface and from the molecules in the thin surrounding gas phase of the liquid jet. All electrons created in the liquid beyond this first nanometer do not reach the detector because of collisions with other molecules [58].

By means of a IR-EUV pump probe approach time resolved photoelectron

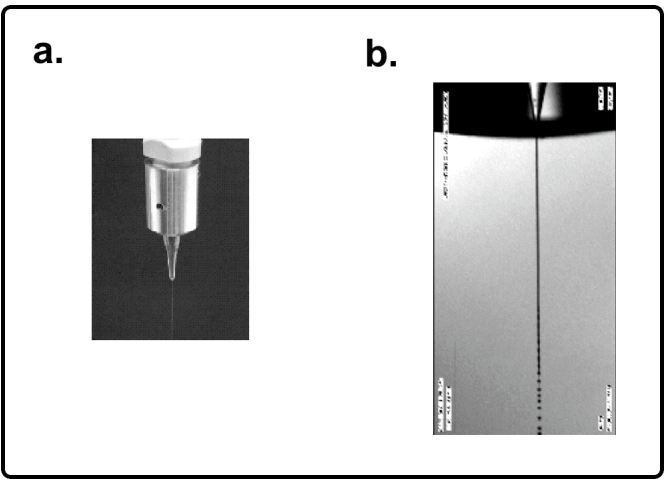

Figure 3.2: a.) Nozzle b.) Liquid beam with diameter of $10 \mu \mathrm{m}$.

spectra of the evolving metastable liquid can be recorded. The time resolution is $\sim 500 \mathrm{fs}$ and the resolution of the recorded photoelectron energies $0.24 \mathrm{eV}$.

The following Chapter provides the theoretical background for the process by which a photoelectron is created and the influences of the surroundings on its binding energy. Furthermore measured spectra of the gas phase and the metastable liquid are presented and discussed.

\subsection{Photoelectron Spectroscopy}

Photoelectrons are generated through the photoelectric effect, which was first discovered by Hertz in 1887 [59]. Einstein gave the theoretical back- 
ground, as he found out that the emission of an electron is only possible, when the energy of the photon is larger than the binding energy of the electron. The difference between binding and photon energy equals the kinetic energy of the emitted electron [60]

$$
h \nu=\Phi+E_{\mathrm{kin}}
$$

where $h$ is the Planck constant, $\nu$ the frequency of the light source, $\Phi$ the work function, which is needed to remove an electron and $E_{\text {kin }}$ the kinetic energy of the emitted electron. For this finding he was awarded in 1905 with the nobel prize for physics.

Although photoelectron spectroscopy is typically used for the analysis from metal surfaces to atoms in the gas phase, in this work the focus lies on the spectroscopy of molecules in the gas and condensed phase. Molecules consists of atoms with electrons, which have different energies depending on the orbital they occupy. Two kinds of electrons can be distinguished: core and valence electrons. The former have a higher binding energy being localized mostly close to the nucleus and are not directly involved in the chemical bonds between atoms. The valence electrons have smaller binding energies and form chemical bonds between atoms. Therefore, they serve as an indicator for binding states of atoms in molecules and also for molecules between each other especially in condensed phases.

The scheme in Figure 3.3 describes the principle of photoelectron spectroscopy on molecules. A high energy photon ejects an electron from its binding valence orbital over the continuum level. The difference between the photon energy and the binding energy of the electron in the molecule is transfered to the electron as kinetic energy. The measurement of this energy using monochromatic light with a specific photon energy yields the binding energy for each electron. Ejecting several electrons of the molecule in the same procedure generates a spectrum, which mirrors the energy dis- 


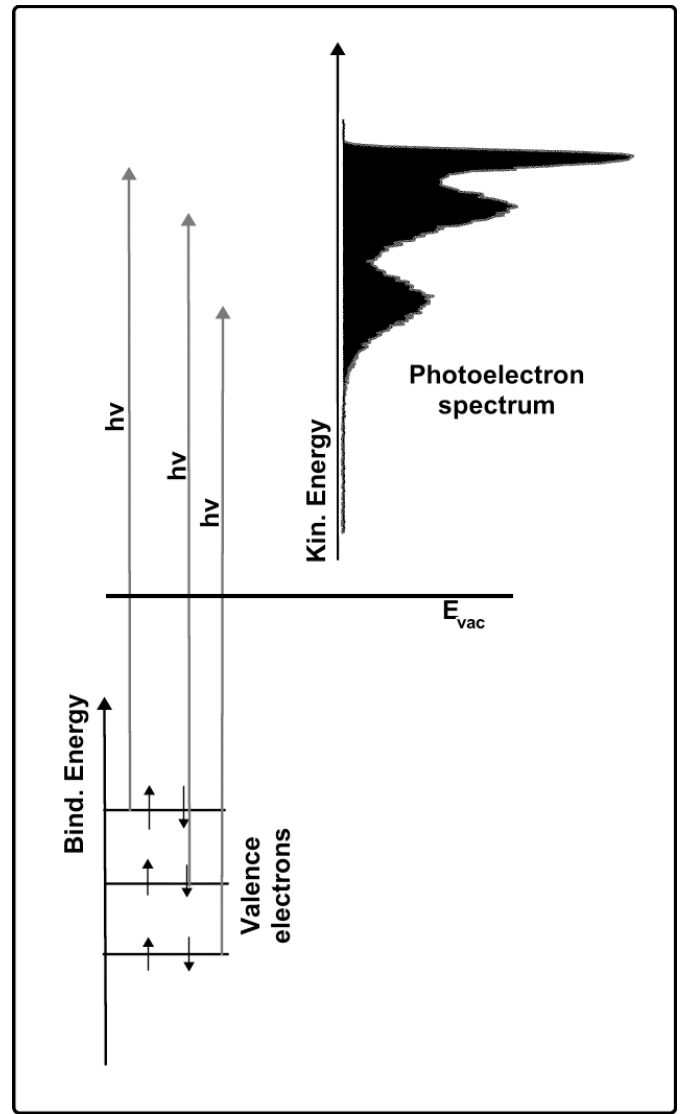

Figure 3.3: Scheme illustrating principles of photoelectron spectroscopy. A high energy photon produces an electron with a kinetic energy, which equals the difference of the photon energy and the binding energy of the electron.

tribution of the electrons in the molecule. For photons with an energy in the vacuum ultraviolet region of the spectrum only valence electrons are ejected.

The process of the ejection of an electron from a molecule A can be described by the following mechanism

$$
\mathrm{A}_{N}+h \nu \longrightarrow \mathrm{A}_{N-1}^{+}+e^{-}
$$


Thereby two different limiting cases can be considered. In the case called sudden approximation the remaining electrons on the molecule are static and their orbitals do not change during the process. The kinetic energy of the electron is the difference between the orbital energy, which is calculated with Koopmans' Theorem (see Chap. 2.4.2), and the energy of the photon. Koopmans' Theorem yields an access to the binding energies measured in photoelectron spectra using quantum chemical calculations like HartreeFock or higher level ab-initio techniques.

The other limiting case is called adiabatic approximation. In contrast to the first one, here the remaining electrons of the ionized molecule are allowed to relax after the ionization process. In this case Koopmans' Theorem is no longer valid, and the kinetic energy of the ejected electron is the energy difference between the ionization potential of the neutral molecule and the photon energy. The calculation of the ionization potential requires the energy of the neutral and ionized molecule and is therefore computationally more demanding. Binding energies calculated with the adiabatic approximation are always larger than energies of the sudden approximation.

With these two approximations binding energies of electrons in molecules can be calculated. However, the measurement of a photoelectron also depends on the probability by which this electron is ejected. One way to calculate this probability is Fermi's Golden rule using the dipole approximation. The transition probability of a system from the initial state $i$ to a final state $f$ is proportional to the coupling strength $M_{f i}^{2}$ between the two states and the density of states of the final state $\rho_{f}$

$$
P_{\text {trans }}=\frac{2 \pi}{\hbar} M_{f i}^{2} \rho_{f}
$$

The coupling strength between the two states is called the matrix element for the transition and equals the integral over the perturbation operator $H^{\prime}$ 
acting on initial state $\psi_{i}$ times $\psi_{f}^{*}$.

$$
M_{f i}=\left\langle\psi_{f}\left|H^{\prime}\right| \psi_{i}\right\rangle
$$

The initial wave function $\psi_{i}$ can be represented as the product of the wave function of the electron which will be ejected $\phi_{e, i}$ and the wave function for the rest of the electrons $\psi_{i, N-1}$. If the same approach is used for the final wave function $\left(\phi_{e, f}, \psi_{f, N-1}\right)$ the square of the transition matrix element can be written as

$$
M_{f i}^{2}=M_{e, f i}^{2}\left\langle\psi_{N-1, f} \mid \psi_{N-1, i}\right\rangle^{2} \rho_{f}^{2}
$$

$M_{e, f i}$ is an one electron transition matrix element and $\left\langle\psi_{N-1, f} \mid \psi_{N-1, i}\right\rangle^{2}$ is called the Franck-Condon factor. When performing single molecule photoelectron spectroscopy, this Franck-Condon factor is responsible for the fine structure of photoelectron spectra. The observed transition are modulated by the overlap of the vibrational and rotational part of the total wave function. Accordingly, an identification of molecular vibrational and rotational modes and their occupation under certain thermal conditions is possible.

As an example, Figure 3.4b shows the photoelectron spectrum of gas phase water recorded with the experimental setup described in 3.1. The binding energy of the electrons is represented on the abscissa and the number of recorded electrons on the ordinate. Three different bands with binding energies ranging from $-12 \mathrm{eV}$ to $-22 \mathrm{eV}$ and different shapes can be distinguished. Figure 3.4a shows a scheme of the five molecular orbitals of a water molecule build up by the atomic orbitals of the respective atoms. Each orbital is assigned a name according to its irreducible representation of the water $C_{2 v}$ symmetry group. The three highest orbitals $\mathbf{1}_{\mathbf{1}}, \mathbf{3} \mathbf{a}_{\mathbf{1}}$ and $\mathbf{1}_{\mathbf{2}}$ are observed in the photoelectron spectrum. The orbital $\mathbf{1}_{\mathbf{1}}$ at $-13 \mathrm{eV}$ is narrow because of its nonbonding character and therefore no vibrational fine structure is present. The other two orbitals $\mathbf{3} \mathbf{a}_{\mathbf{1}}$ and $\mathbf{1} \mathbf{b}_{\mathbf{2}}$ are involved 
a)

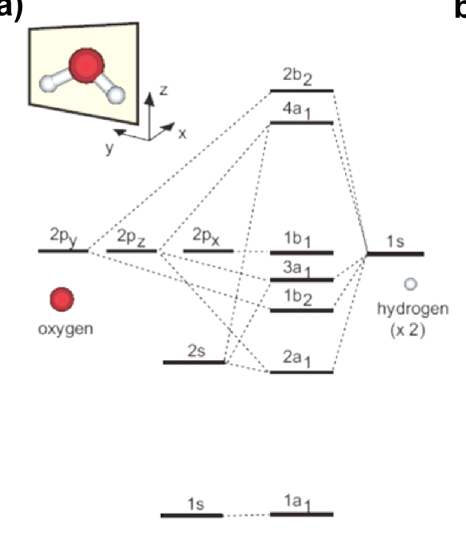

b)

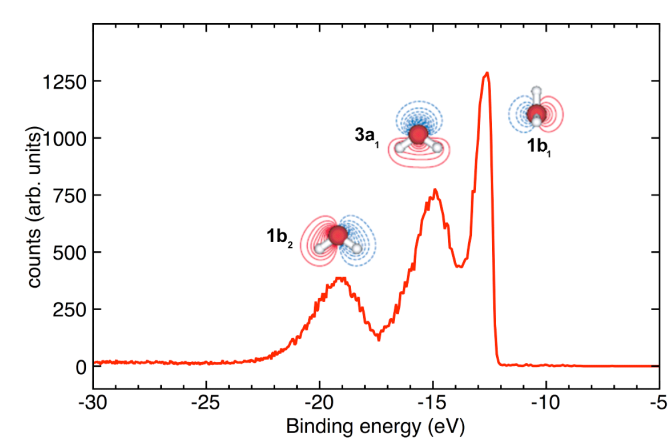

Figure 3.4: a) Molecular orbitals of the water monomer. b) Spectrum of gas phase water recorded with the experimental setup described in Chapter 3.1.

in the oxygen hydrogen bond and exhibit therefore a broad band in the spectrum. However, single vibrational transitions can not resolved because of the low resolution of the experimental setup.

In condensed phases several factors which change the appearance of the spectra may shift the kinetic energy of the electrons. Especially the influence of the surrounding molecules and their charge distribution changes the binding energy of the electrons. Scheme 3.5 illustrates the influences on the involved species. Surrounding molecules can stabilize the neutral initial state, the ionized final state or both. In the following different contributions to the stabilization will be presented and discussed.

Surface potentials: Photoelectron spectroscopy on liquids forces the ejected electrons to overcome the liquid-vacuum boundary in order to reach the detector. This boundary possess an electrostatic potential, which can induce a shift in the kinetic energy of the electrons. For the liquid jet, which is used in this work (see description in 3.1), this potential was measured by 


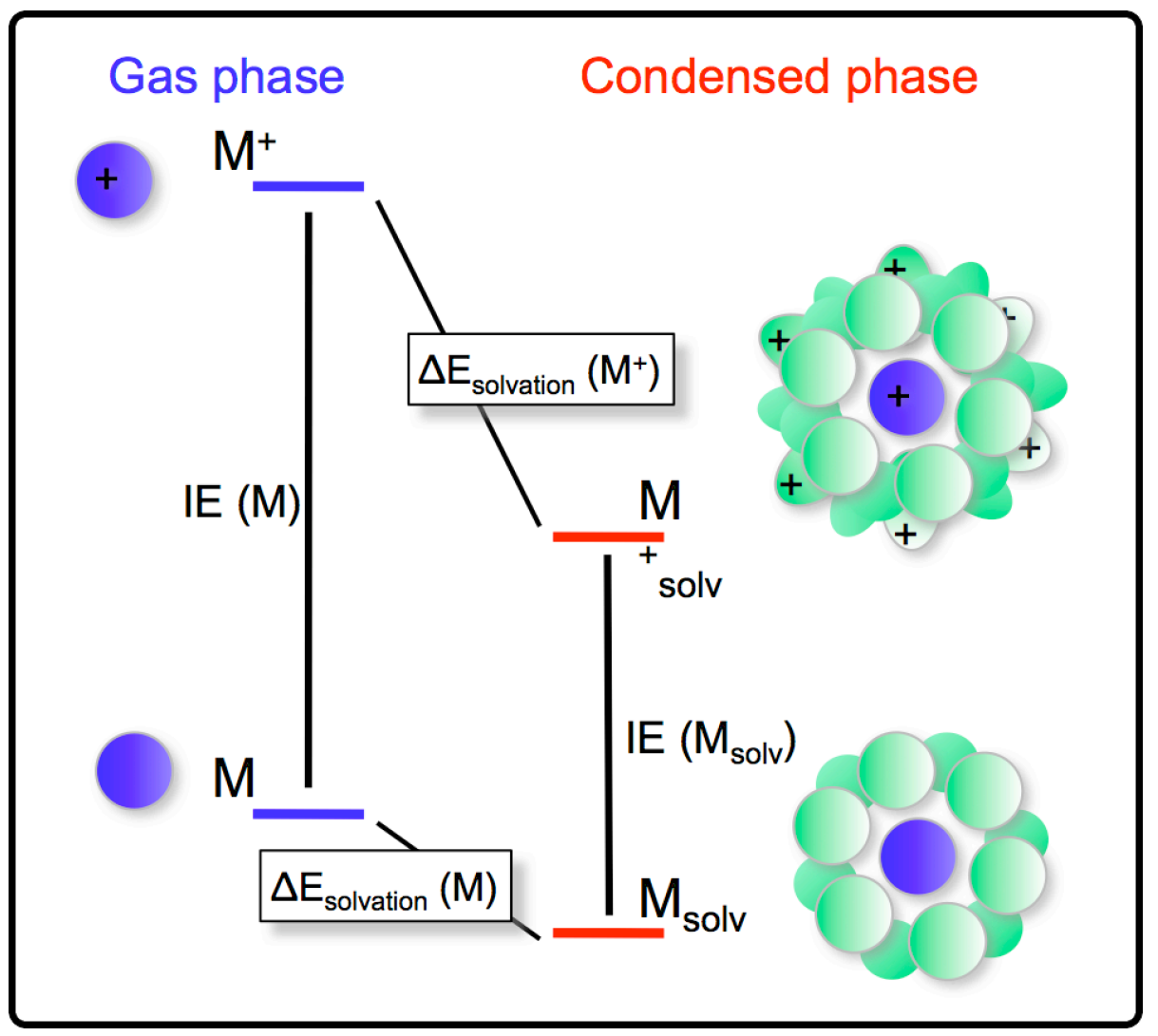

Figure 3.5: Scheme representing the influences of the condensed phase on the ionization potential.

Faubel et al. [58] to be smaller than $10 \mathrm{meV}$. This small value agrees with the simulations of Mundy et al. [61] who finds the water dipoles oriented along the water-vacuum interface. Because of the small value the effect on the recorded photoelectron spectra can be neglected.

Solvation effect: Moving a molecule or an ion from the gas into the liquid phase is associated with a change in energy. This change can be split into different contributions being $\Delta E_{\text {Coul }}$ the Coulombic contribution, $\Delta E_{\mathrm{Pol}}$ the contribution caused by a 
polarization, $\Delta E_{\text {Disp }}$ the contribution of dipol-dipol interactions and $\Delta E_{\text {Exch }}$ the contribution due to the repulsion of electron clouds.

$$
\Delta E=\Delta E_{\text {Coul }}+\Delta E_{\text {pol }}+\Delta E_{\text {Disp }}+\Delta E_{\text {Exch }}
$$

Agren et al. [62] describes a method for the calculation of the different terms for one atom $A$ using the radial distribution function $g_{A, B}\left(\mathbf{R}_{\mathbf{A}, \mathbf{B}}\right)$.

$$
\begin{aligned}
\Delta E_{A}= & \sum_{B}^{N} \int_{0}^{\infty} g_{A, B}\left(\mathbf{R}_{\mathbf{A}, \mathbf{B}}\right) . \\
& {\left[\frac{q_{B}}{\mathbf{R}_{A, B}}-\frac{\left(Q_{f}-Q_{i}\right) \tilde{P}_{B}}{2 \mathbf{R}_{A, B}^{4}}-\frac{A_{B}}{\mathbf{R}_{A, B}^{6}}+\frac{B_{B}}{\mathbf{R}_{A, B}^{12}}\right] }
\end{aligned}
$$

The interaction of all atoms around A is summed up and integrated over the radial distribution function times the different contributions. $Q_{f}$ and $Q_{i}$ are the charges of the atom A after and before ionization. $\tilde{P}_{B}$ is the spherical average polarizability of atom $B$ and $A_{B}$ and $B_{B}$ the dispersive and exchange coefficients. With some approximations especially in the Coulomb interactions (because of their long range character) and neglecting the dispersion and exchange contributions Agren was able to derive shifts for solvated cations and anions and achieved good agreement with experiment [62].

A further approximation can be made if the polarization effects of the solvent due to the solute are also neglected. The energy difference between gas and solvated species is then given by the Born energy of solvation which accounts for the energetic stabilization of a charge particle $I$ with an Onsager cavity $a$ in an 
average electrostatic field generated by a solvent with a dielectric constant $\epsilon$.

$$
\Delta E_{I}=-\frac{e^{2} q_{I}^{2}}{2 \cdot 4 \pi \epsilon_{0} a}\left(1-\frac{1}{\epsilon}\right)
$$

Since the ejection of the electron is a vertical process and the solvent is not able to relax during the process only the optical part of the dielectric constant $\epsilon$ can be used $\left(\epsilon_{\mathrm{opt}}=1.8\right)$. But still the Onsager cavity a remains an unknown parameter. Furthermore, this approximation accounts only for the stabilization of the ionized charged state and neglects any stabilization of the neutral initial state.

Although the solvation of the initial and final state represented through the mentioned methods may recover large contributions of the solvation energy, they do not account for the local time dependent structure of the surrounding molecules, which may also change the binding energies of the electrons [63]. Shifts in binding energy becomes more pronounced if hydrogen bonded liquids are studied as in this work.

Hydrogen bonds: Local time dependent configuration of the surrounding neighbors play a major role especially in hydrogen bonded systems. Agren [63] included these effects one year later in a supermolecular approach in which the first solvation shell was treated with quantum chemical methods and the environment in a continuum model. For the dimer in vacuum using the Hartree-Fock method he found an energy shift of $-1.11 \mathrm{eV}$ for the acceptor molecule and $0.77 \mathrm{eV}$ for the donor. This shift could be mostly assigned to the electrostatic interactions with a little contribution of polarization effects and charge transfer. But he concluded that this energy shifts were not able to reproduce the energy shifts observed between the gas and the 
ice or the liquid phase. His next step was to include the nine nearest neighbors at the cubic ice conformation, which lead to an extra energy shift of $-0.3 \mathrm{eV}$. Only with correction for surface effects of the cluster and a statistic average distribution of the environment he could predict the energy drift for liquid water of the highest occupied molecular orbital (HOMO) to be $-1.94 \mathrm{eV}$. But still this approach suffered from the exclusion of dynamical effects rising from different conformations found especially in liquids with hydrogen bonds like water and methanol. Faubel et al. measured the energy shift of the HOMO in liquid water to be $-1.45 \mathrm{eV}[58]$.

A similar approach was used by Winter et al. [9] where the shift of the core electron binding energies of liquid water could be assigned to changes in the distance and the angle of the hydrogen bonded neighbors. He reported a negative shift as a consequence of a shortening of the distance between the acceptor hydrogen bonded molecules and a positive energy shift by a shortening of the donor bonded ones. For liquid water at surfaces $[64,8]$ and in the bulk [3] the energy shifts of the core electrons helped the corresponding authors to identify and quantify the hydrogen bonded species as double donor, single donor, single acceptor or double acceptor.

From the presented studies it can be concluded that hydrogen bonds have influences on the energy shifts of ejected electrons and that their change is correlated with a change in the appearance of photoelectron spectra.

As an example of the influence of the environment on the photoelectron spectrum, a comparison of the spectrum of gas phase and liquid water is shown in Figure 3.6. Liquid water shown in blue possesses broader bands in the binding energy region of the $\mathbf{1}_{\mathbf{2}}$ and $\mathbf{3} \mathbf{a}_{\mathbf{1}}$ orbitals of the gas phase 


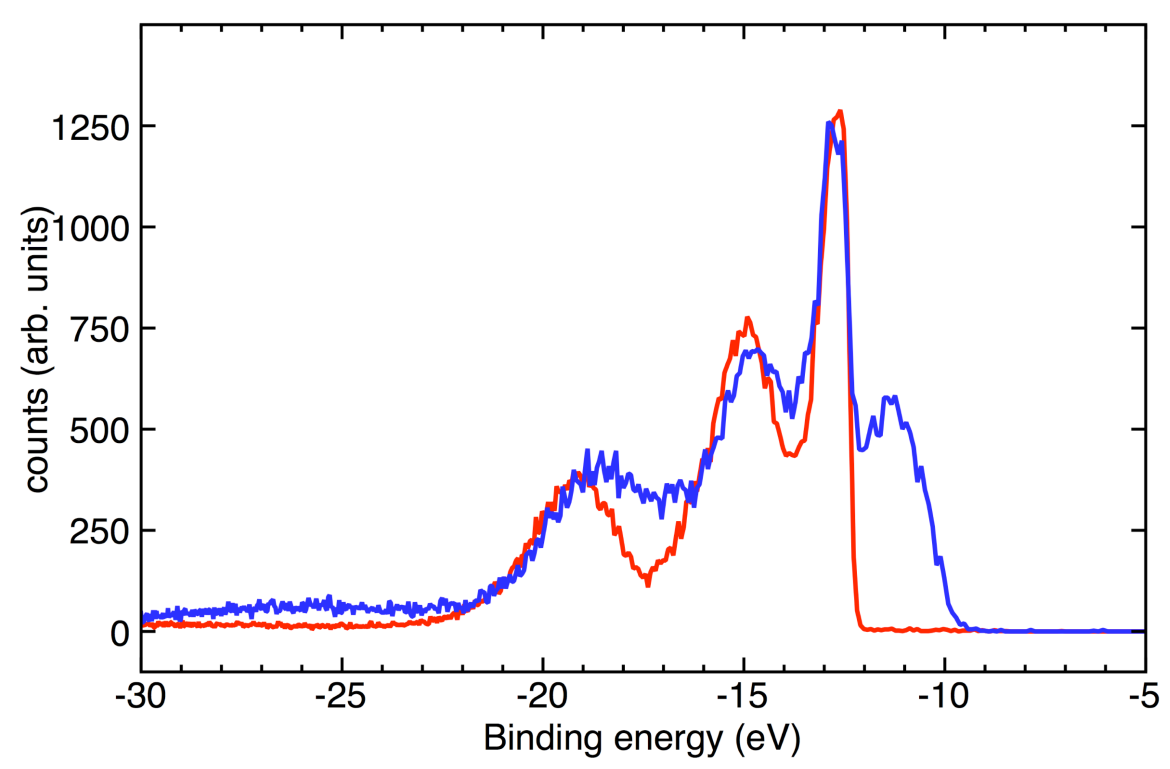

Figure 3.6: Photoelectron spectra of gas phase water (red) and liquid water (blue).

spectrum, which can be explained by new intermolecular vibrations in the liquid phase. Furthermore, a new band with binding energies smaller than the HOMO orbital of gas phase water are distinguished $\left(\mathrm{E}_{\text {bind }}=-12-\right.$ $10 \mathrm{eV})$. This new band arises from electrons which energy is lower than their respective energy in the monomer. This destabilization stems from a destabilization of the ground state or a stabilization of the ionic state (see Fig. 3.5). In the former case the destabilization is produced by a decrease of binding energy of the HOMO electrons which is a result of hydrogen bonds where the acceptor is destabilized because of the lost of electron density to the donor. In the latter the stabilization of the ionic state is caused by long range electrostatic and dipol-dipol stabilization in condensed phases.

Because photoelectron spectra are so sensitive to changes at the molecular level between the liquid and the gas phase they provide an access to study the dynamics of metastable liquids: After a high energy deposition on a liquid under normal conditions as described in Chapter 3.1 time de- 
pendent photoelectron spectra are recorded. These spectra allows to trace the macroscopic process and to correlate them with changes in the environment at a molecular level. Thereby, the appearance and the dynamics of the spectra may differ for different liquids.

Experimental spectra were obtained for two different liquids: water and methanol [11]. Methanol was chosen because of the similarity to water in respect to molecular geometry and size. The main change consist in an exchange of a hydrogen atom with a $\mathrm{CH}_{3}$ group. This causes differences in the rotational constants because of the higher mass of the $\mathrm{CH}_{3}$ group and in the electrostatic interactions of the molecule since the more electronegative carbon has a less positive partial charge than the hydrogen atom. This smaller charge does not only change the number of possible hydrogen bonds in respect to water but also their dynamics. However, methanol allows to study experimentally the influence of the hydrogen bonds on the dynamics of metastable liquids without changing the geometrical properties of the molecules.

Figure 3.7 shows time dependent photoelectron spectra for water measured at various deposited energies. For low energy deposition, which corresponds to a temperature of $450 \mathrm{~K}$, the characteristic liquid band disappears on a time scale of $\sim 200 \mathrm{ps}$ at the same time as the gas band gains intensity. In contrast, for high energy deposition, where temperatures of $775 \mathrm{~K}$ are reached, the liquid disappears faster $(\sim 100 \mathrm{ps})$ and later a new band arises with a binding energy between the liquid and the gas phase band. The respective spectra for methanol (Fig. 3.8) - with slightly lower temperatures because of the lower absorption coefficient - show also a decrease in the liquid band but on a longer time scale and for the high energy deposition no new band is formed.

To study the different dynamics of the two liquids and to identify the new species formed in supercritical water, computer simulations of the expansion process were performed. Different species in the simulations will be identified and the evolution of hydrogen bonds will help to understand the 


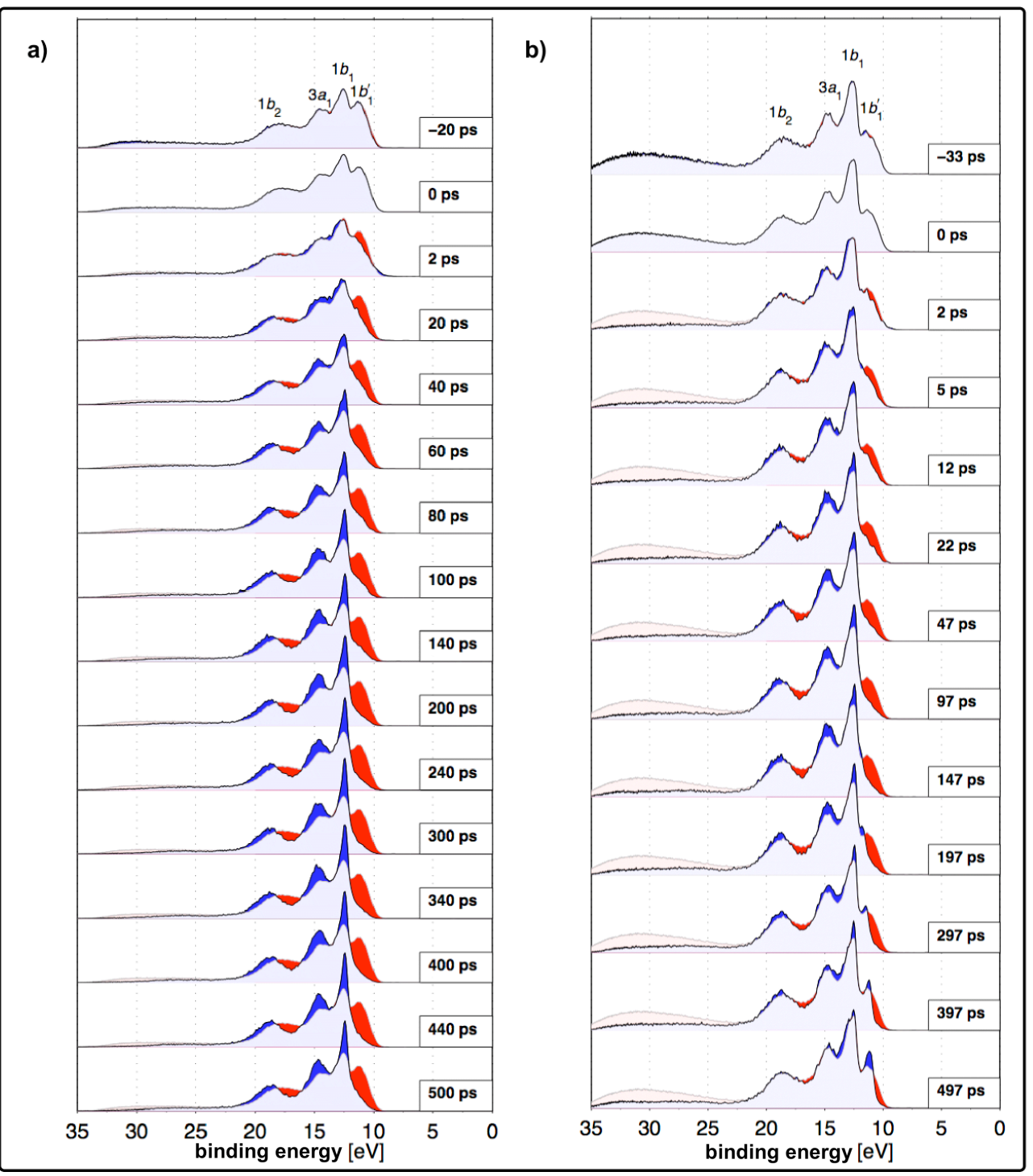

Figure 3.7: Sequence of photoelectron spectra of water for a low energy deposition $(\lambda=2700 \mathrm{~nm})$ and $\mathbf{b}$ high energy deposition $(\lambda=2800 \mathrm{~nm})$ (red: loss of photoelectrons with this binding energy; blue: gain in photoelectrons). 


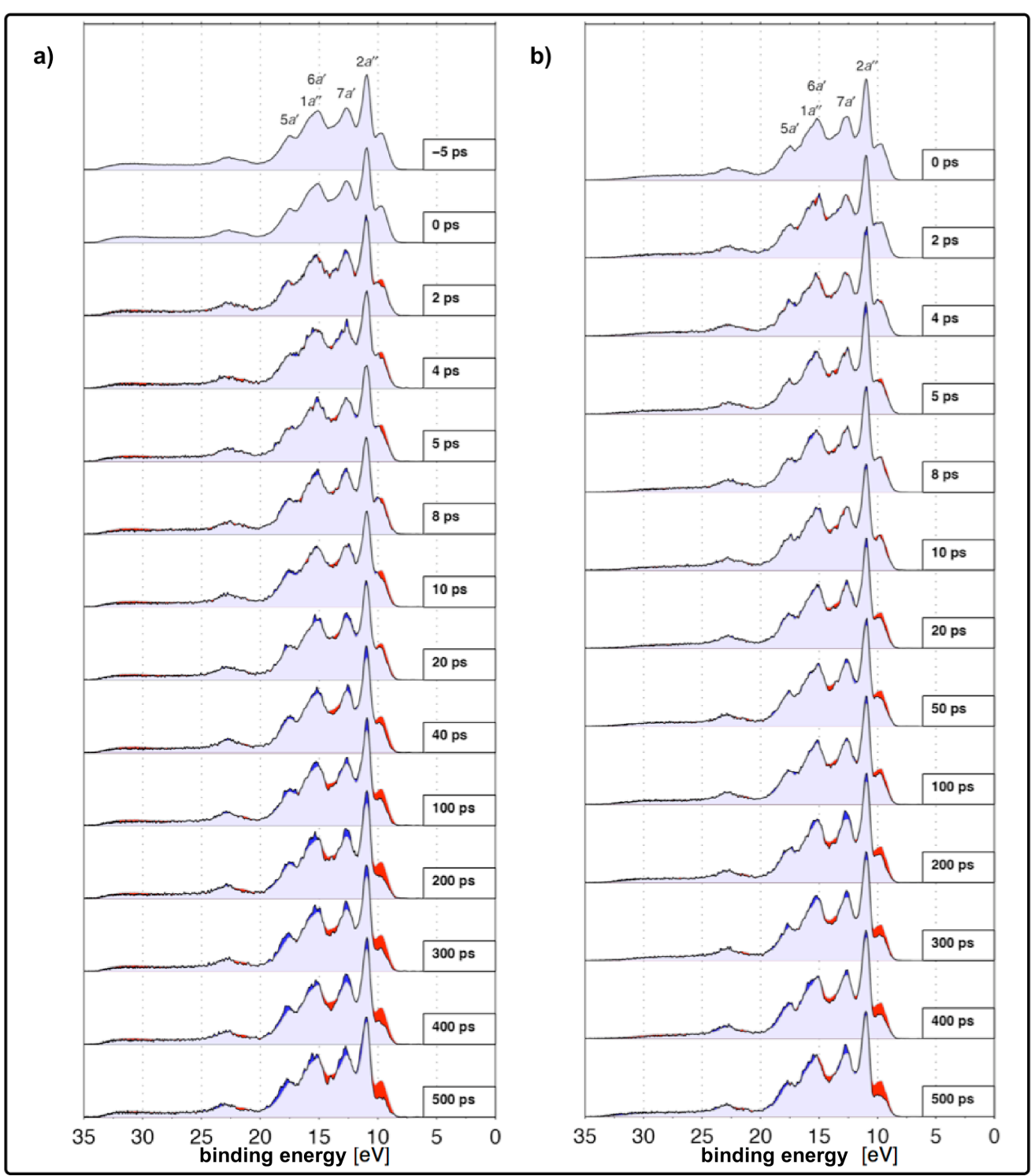

Figure 3.8: Sequence of photoelectron spectra of methanol for a low energy deposition $(\lambda=2900 \mathrm{~nm})$ and $\mathbf{b}$ high energy deposition $(\lambda=3000 \mathrm{~nm})$ (red: loss of photoelectrons with this binding energy; blue: gain in photoelectrons). 
different contributions of the observed spectral signatures.

\subsection{Simulation Setup}

For the simulation of the dynamics of metastable liquids the GROMACS simulation package [33] was used. A rectangular box with dimensions of $30 x 4 x 4 \mathrm{~nm}$ of the corresponding liquid was first equilibrated at $300 \mathrm{~K}$ and 1 bar using the temperature and pressure coupling algorithm of Berendsen [65]. For the simulation of water a total number of 16184 molecules were simulated with four different models - SPC, SPC-E, TIP4P and flexible SPC - to check their influence on the dynamics. For a detailed description of the models the reader is referred to Chapter 2.6.1. Two $\mathrm{NaCl}$ molecules were added to match the experimental salt concentration of $15 \mathrm{mM}$. For methanol the OPLS force field was used since the GROMOS force field does not yield the experimental liquid density of methanol under atmospheric conditions. The time step to integrate Newton's equation was set to $1 \mathrm{fs}$. The short range Coulomb interactions were calculated explicitly until a distance of $1.2 \mathrm{~nm}$ and for the long range interactions the Particle Mesh Ewald method was used [66]. The Van-der-Waals interactions were shifted at a distance of $0.8 \mathrm{~nm}$ and then leveled off to zero at $1.0 \mathrm{~nm}$.

The infrared laser used in the experiment to create metastable liquids excites the $\mathrm{OH}$-stretch vibration of the respective liquids. According to Elsässer et al. [2] the deposited vibrational energy is distributed onto the other vibration and librational modes on a time scale of $50 \mathrm{fs}$ in liquid water. This time scale is on the one hand much shorter than the time resolution achieved in the experiment such that it can not be resolved experimentally and on the other hand this time period is too short to allow an expansion of the liquid. Therefore, the equilibrated system at $300 \mathrm{~K}$ and 1 bar was heated up under constant volume to different temperatures and post 
equilibrated for 200 fs to reach a homogenous temperature distribution over the box and all degrees of freedoms. For water, where in the experiment internal energies of $15 \mathrm{~kJ} / \mathrm{mol}, 30 \mathrm{~kJ} / \mathrm{mol}$ and $45 \mathrm{~kJ} / \mathrm{mol}$ were achieved, the corresponding temperatures were $450 \mathrm{~K}, 775 \mathrm{~K}$ and $1100 \mathrm{~K}$. The experimental internal energies for methanol were slightly lower because of the lower absorption coefficient at this wavelength. However, comparable temperatures of $500 \mathrm{~K}, 650 \mathrm{~K}$ and $800 \mathrm{~K}$ as for water were employed to elucidate differences between the two liquids at extreme temperatures.

After reaching the respective temperatures, the simulation box was extended to $500 \mathrm{~nm}$ and the heated liquid centered in the middle. Four to seven production runs were obtained from simulations of this enlarged box at constant volume and energy for 100 ps with the mentioned parameters. During those simulations the heated liquid was allowed to expand on both sides, such that two independent expansion processes per simulation were analyzed. For each liquid and temperature four to seven systems were simulated, built from independent configurations extracted from the heating up process. To check for finite size effects, a larger water system of $12 \times 12 \times 30 \mathrm{~nm}$ with $\sim 430000$ atoms at $775 \mathrm{~K}$ was also simulated and analyzed.

\subsection{Results}

The results of the simulations are shown as representative snapshots revealing the main features. Afterwards, analysis concerning density, hydrogen bonds and the formation of aggregates are presented.

Figure 3.9 displays snapshots at different times for the large water simulation system $\left(\sim 4.3 \cdot 10^{6}\right.$ atoms $)$ equilibrated at $775 \mathrm{~K}$. All snapshots show the left side of the simulation system with a total length of $\sim 130 \mathrm{~nm}$. The water molecules are colored according to the phase diagram of water (Fig. 3.10). 


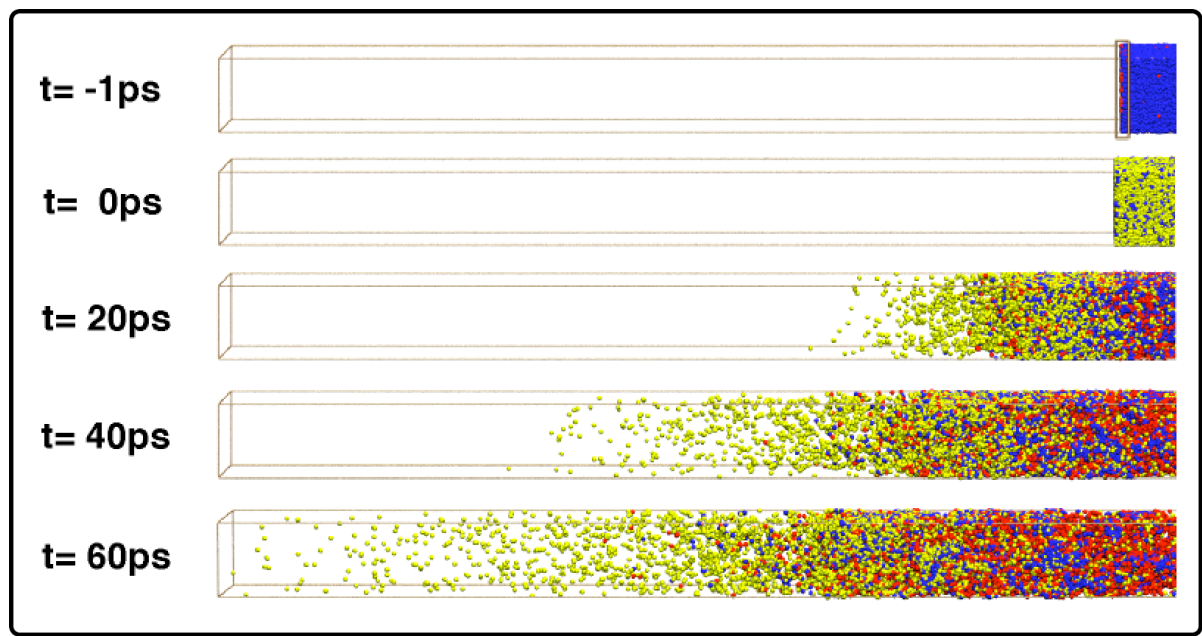

Figure 3.9: Snapshots at different times for the simulation of metastable water at $775 \mathrm{~K}$. Molecules are colored according to phase diagram shown in Figure 3.10.

Thereby, the averaged values over the time period of $2 \mathrm{ps}$ of the kinetic energy of each molecule and the number of neighbors in a sphere $(\mathrm{r}=3.5 \AA)$ around each water molecule served to calculate its temperature and density. The obtained density and temperature were used to compute the pressure with the equation of state for water from Kell et al. [67]. Each temperature and pressure value pair of a water molecule falls in a region of the phase diagram (Fig. 3.10) and allowed a qualitative description of the evolution of the metastable liquid created at supercritical fluid conditions. However, this procedure assumes a local equilibrium for each water molecule on this time scale, which may not be the case in the fast expansion process.

The top snapshot in Figure $3.9(t=-1 \mathrm{ps})$ shows the equilibrated liquid water system (blue) at $300 \mathrm{~K}$ and 1 bar. The $1 \mathrm{~nm}$ wide box at the vacuum liquid interface encloses the water molecules which electrons contribute to the photoelectron spectra (see Chapter 3.1). The following snapshot is taken after heating up the system at constant volume to the condition of a supercritical fluid (yellow) and represents the beginning of the production runs. The simulation snapshots at 20,40 and 60 ps show a fast dispersion of 


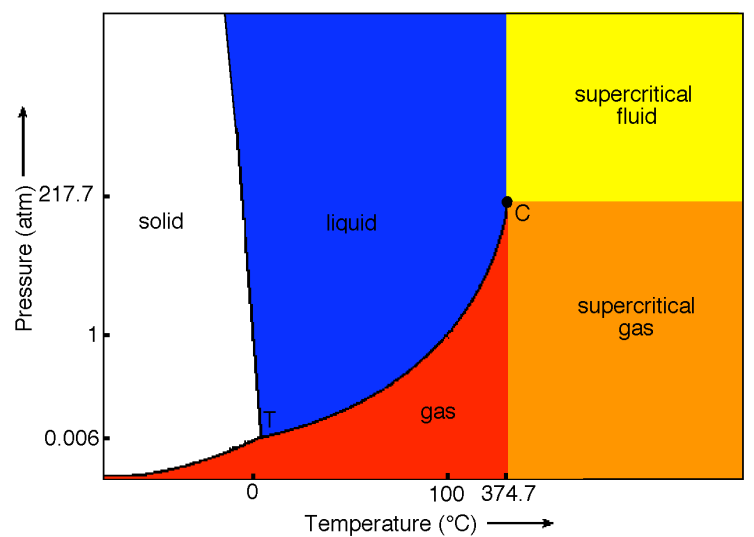

Figure 3.10: Phase diagram of water.

the supercritical fluid in single water molecules and aggregates, which still belong to the supercritical fluid phase because of their high kinetic energy and density. All simulation systems tempered at $775 \mathrm{~K}$ and at $1100 \mathrm{~K}$ show the same characteristic dispersion into single molecules and aggregates independently of the system size or the water model. At higher temperatures the dispersion becomes faster and more aggregates are formed.

In contrast to the supercritical metastable water simulations the water

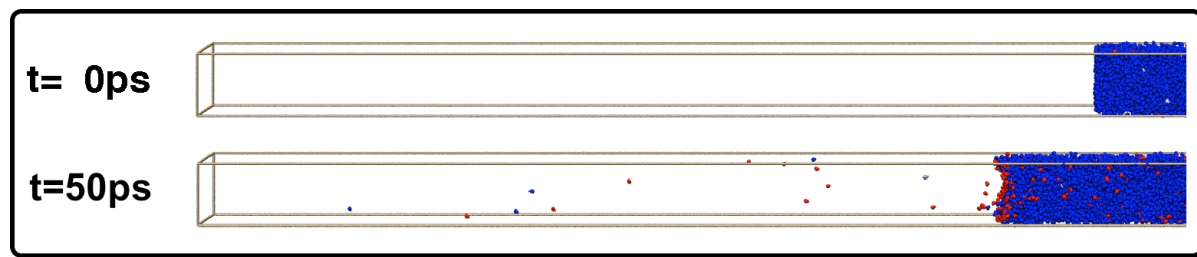

Figure 3.11: Snapshots at different times for the simulation of subcritical water at $450 \mathrm{~K}$. Molecules are colored according to phase diagram shown in Figure 3.10.

systems under subcritical conditions $(\mathrm{T}=450 \mathrm{~K})$ displayed in Figure 3.11 do not indicate any dispersion and only few molecules leave the fluid at all. This behavior can be interpreted as an evaporation process where single molecules at the interface get enough energy to leave the fluid and con- 
tribute to the gas phase.

For methanol, the simulations were performed in the same way as for water. Simulations with initial temperatures lower than the critical temperature (Figure 3.12 top) exhibit the same behavior as the subcritical water simulations. The supercritical methanol simulations at $650 \mathrm{~K}$ and $800 \mathrm{~K}$ $\left(T_{\text {crit }}=512 \mathrm{~K}\right)$, however, reveal in contrast to water no formation of small aggregates. Only some monomers depending on temperature are observed on this time scale (Figure 3.12 bottom). This result agrees very well with the experimental finding where no new band forms at later times (see Figure 3.8).

This outcome indicates that water at supercritical conditions has different properties than methanol in respect to the expansion process. In Chapter 3.4.3 the differences will be interpreted in terms of rates for formation and dissociation of hydrogen bonds which represents the first step in the dispersion of a liquid.

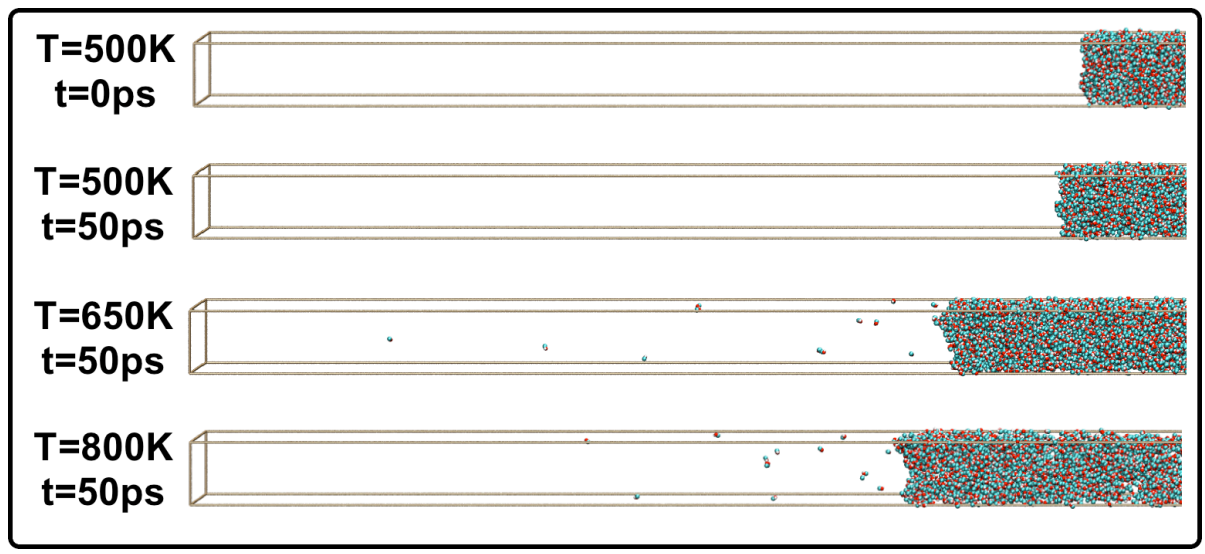

Figure 3.12: Snapshots of subcritical and supercritical methanol simulations for different initial temperatures. 


\subsubsection{Density}

One of the thermodynamic properties which is expected to reveal pronounced changes during the evolution of metastable liquids is their density. At the beginning of the expansion process the density corresponds to the density at normal conditions $\left(\rho_{298 \mathrm{~K}, 1 \mathrm{bar}}\left(\mathrm{H}_{2} \mathrm{O}\right)=997 \mathrm{~g} / \mathrm{dm}^{3}\right)$ and should tend to zero for later times, when molecules has only neighbors at very large distances. The change in density might also be reflected in the measured photoelectron spectra, since lower numbers of neighbors change the stabilization of the neutral and the ionized state in the vertical ionization process (Chapter 3.2).

To obtain the average density around a molecule at each time step the radius of a sphere containing the next six neighbors of each water molecule at a certain time $t$ was recorded. The analysis was performed for molecules which contribute to the experimental spectra. At the beginning of the simulation the number of analyzed molecules add up to the ones contained in the $1 \mathrm{~nm}$ wide box shown Figure 3.10. For later times the same number of molecules were analyzed counting the first molecules from the vacuum side to the liquid. The radius of the sphere of each molecule was averaged over all molecules and over four to seven independent simulations for each picosecond. With the obtained radius the density $\rho$ for water was calculated via the following equation

$$
\rho=\frac{N_{\mathrm{H}_{2} \mathrm{O}} 55.55}{N_{\mathrm{A}} \frac{4}{3} \pi r^{3}} \text {. }
$$

$N_{\mathrm{H}_{2} \mathrm{O}}$ represents the number of water molecules in the sphere, $N_{\mathrm{A}}$ the Avogradro number, $r$ the radius of the sphere in $\mathrm{dm}^{3}$ and $\rho$ is given in $\mathrm{g} / \mathrm{dm}^{3}$. The averaged densities for water as a function of time are displayed in Figure 3.13 for the three initial conditions. The density at $\mathrm{t}=0 \mathrm{ps}$ corresponds independently of the temperature to the density at normal conditions. The density of the simulations at supercritical temperatures of $775 \mathrm{~K}$ (green) 


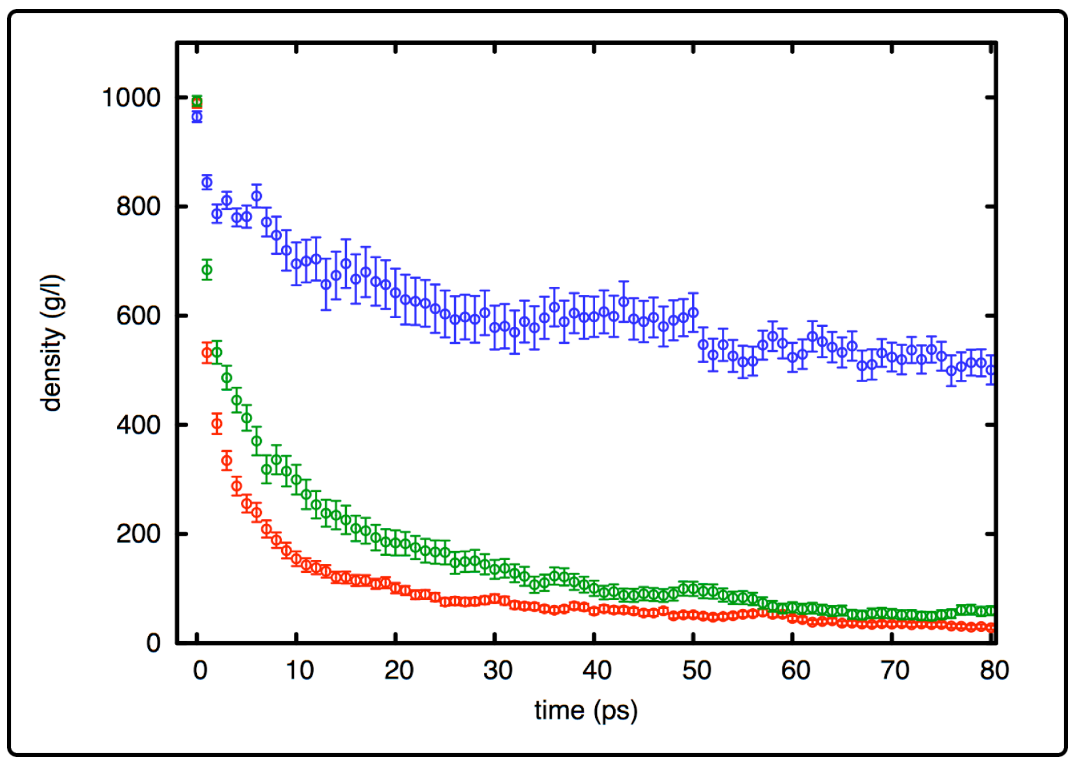

Figure 3.13: Average density as a function of time for water simulations performed with the SPC model at different initial temperatures (blue $=450 \mathrm{~K}$, green $=775 \mathrm{~K}$ and red $=1100 \mathrm{~K})$.

and $1100 \mathrm{~K}$ (red) drops by one order of magnitude on a time scale of 20$40 \mathrm{ps}$. The subcritical simulations at $450 \mathrm{~K}$ drops on a much longer time scale and seems to reach a plateau of $\sim 500 \mathrm{~g} / \mathrm{dm}^{3}$.

Compared to the measured time scales in which the liquid band in the experiment disappears ( $\sim 100 \mathrm{ps})$ the drop in density seems too abruptly and too fast. To exclude the water model being responsible for the discrepancy the supercritical simulation at $775 \mathrm{~K}$ was performed also with different models. The models employed were: SCP/E, TIP4P and SPC-Flex. The differences constitute in inclusion of an average polarization to the total energy (SPC/E), a fourth interaction site (TIP4P) and the inclusion of the vibrational degrees of freedoms in the SPC model (SPC-Flex) (a detailed description of water models is described in Chapter 2.6.1). The densities as a function of time are displayed in Figure 3.14 in comparison to the used SPC model. As can be seen all models except for the TIP4P model revealed 


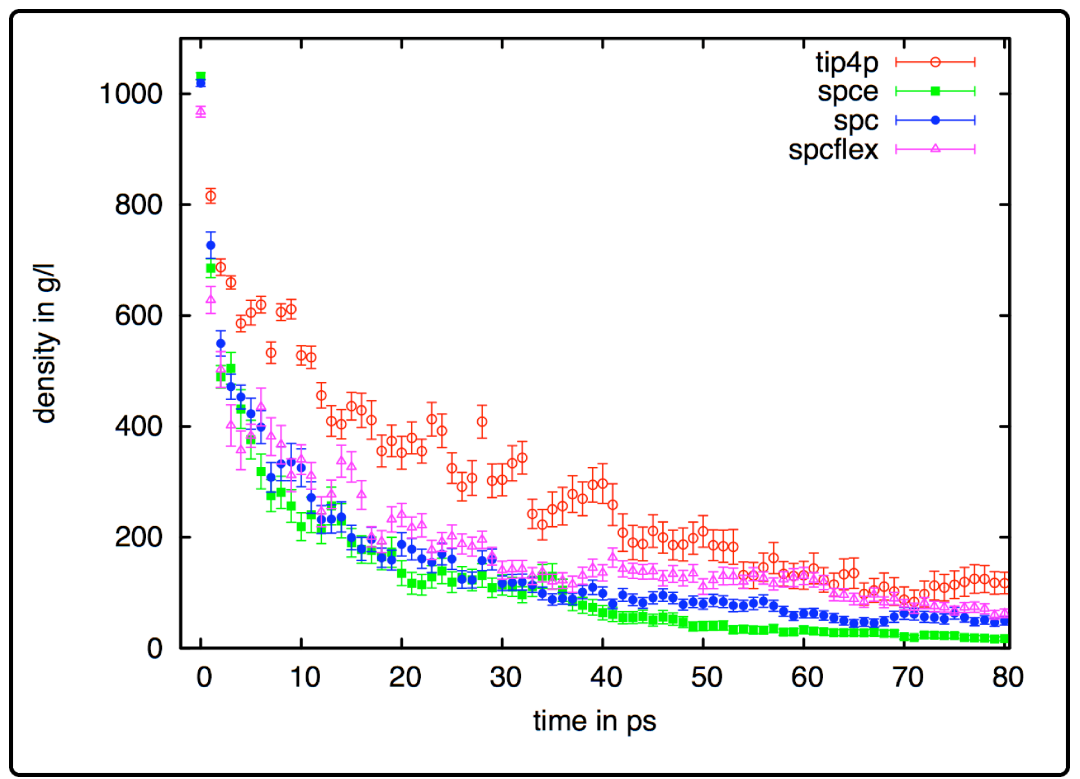

Figure 3.14: Average density as a funtion of time for water simulations with different water models at a temperature of $775 \mathrm{~K}$.

the same density dependency. The deviation of the TIP4P model can be explained by the different charge distribution which may change the overall expansion process slightly but still display the same qualitative trend.

\subsubsection{Hydrogen Bonds}

As discussed previously, not only the density around a molecule might influence the photoelectron spectra. Especially in liquids like water and methanol hydrogen bonds play an important role. Stronger interactions between two molecules due to hydrogen bonds result in large changes in the molecular orbitals of the acceptor and donor molecule producing shifts in the photoelectron spectrum. The magnitude of the observed shift can be correlated with the strength of the hydrogen bond.

To elucidate the dynamics of the involved hydrogen bonds during the ex- 
pansion process of water and methanol the respective simulations were analyzed in respect to changes in the energy and the number of hydrogen bonds. A geometric definition for the hydrogen bonds was employed and its energies were calculated with the Espinosa equation (see Chapter 2.7). Each configuration of atoms at time $t$ in a hydrogen bonded liquid has a characteristic distribution of hydrogen bond energies rising from different hydrogen bonded molecules. The average distribution at $300 \mathrm{~K}$ and 1 bar was used to obtain the average hydrogen bond energy of one molecule. This energy divided by the number of hydrogen bonds per molecule $\left(\bar{N}_{\mathrm{H}_{2} \mathrm{O}}=1.7, \bar{N}_{\mathrm{MeOH}}=1.06\right)$ yielded the average energy of a hydrogen bond of the respective liquid $\left(E_{\mathrm{H}_{2} \mathrm{O}}(\mathrm{OH} \cdots \mathrm{H})=15.5 \mathrm{~kJ} / \mathrm{mol}\right.$, $\left.E_{\mathrm{MeOH}}(\mathrm{OH} \cdots \mathrm{H})=29.9 \mathrm{~kJ} / \mathrm{mol}\right)$. To study the dynamics of the expansion process, at each picosecond the hydrogen bond energy distribution of four to seven trajectories were averaged and divided by this average hydrogen bond energy to reflect changes in the number of comparable hydrogen bonds. Figure 3.15 displays the change of this number during the expansion process for water and methanol at different initial temperatures. The supercritical water simulations (Fig. 3.15a,b) show major changes in the first picoseconds but the average number of comparable hydrogen bonds (thick black line) remains almost constant. At the beginning water molecules with small number of hydrogen bonds corresponding to small binding energies vanish and after $\sim 50 \mathrm{ps}$ the whole distribution is shifted to smaller numbers of comparable hydrogen bonds. This changes in the distribution leads to the conclusion that the expansion process is accompanied with the a change in the population of hydrogen bonds of the involved molecules. The fraction of water molecules which are not hydrogen bonded (NHW) (lower panel of each plot) increases during the expansion process of the supercritical simulations. However, after 100 ps $40 \%$ of the water molecules are still involved in hydrogen bonds at $775 \mathrm{~K}(\sim 30 \%$ after $50 \mathrm{ps}$ at $1100 \mathrm{~K})$.

In contrast, the subcritical water simulations and the supercritical methanol simulation reveal only minor changes during the simulation (Fig. 3.15c,d). 


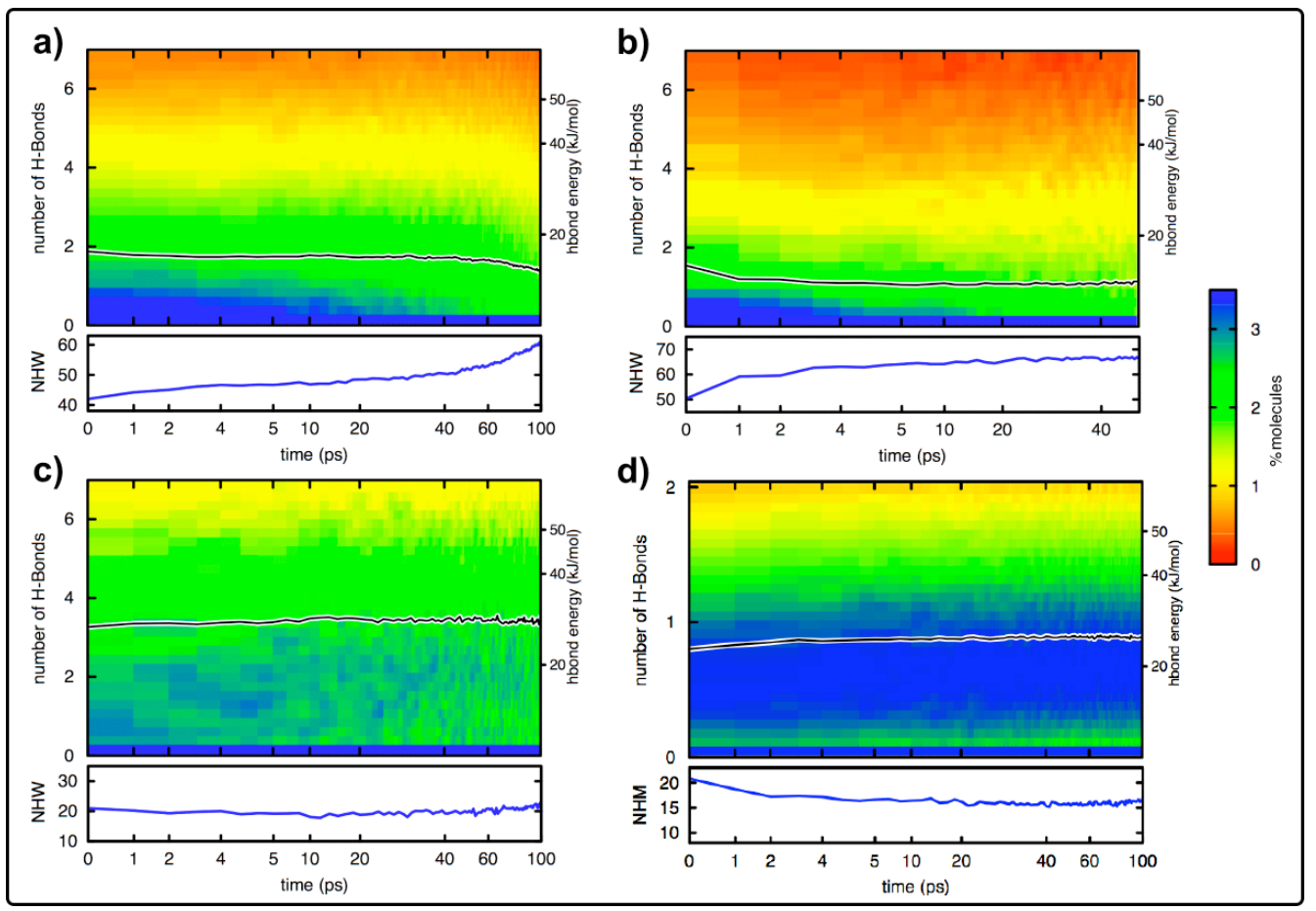

Figure 3.15: Fraction of molecules with a certain energy or number of hydrogen bonds as a function of time during expansion process. Lower panel: Percentage of not hydrogen bonded molecules (NHW/NHM). a-c) Water simulations with initial temperatures of $775 \mathrm{~K}, 1100 \mathrm{~K}$ and $450 \mathrm{~K} \mathrm{~d}$ ) Methanol simulations with a temperature of $650 \mathrm{~K}$.

For subcritical water the average number of comparable hydrogen bonds per water molecule as well as the number of water molecules with no hydrogen bonds are almost constant during the simulation time of $100 \mathrm{ps.} \mathrm{For}$ supercritical methanol the average number of hydrogen bonds per molecule at the beginning was smaller than for water because methanol has only one $\mathrm{OH}$ group to build a hydrogen bond. In contrast to supercritical water the number increased slightly during the simulation. This difference to supercritical water may be interpreted as a change from chain like structures found in methanol under high density conditions to ring like configurations favorable at lower densities which enable the formation of stronger hydro- 
gen bonds [49]. The fraction of not hydrogen bonded methanol molecules (NHM) decreased by $5 \%$.

\subsubsection{Hydrogen Bond Dynamics}

As denoted in the Chapter before, the expansion process at supercritical conditions is accompanied by a change in the nature of the involved hydrogen bonds. Especially in the comparison of the supercritical water and methanol simulations, water exhibits a severe change in the hydrogen bonds energetics in contrast to supercritical methanol.

To study the different hydrogen bond dynamics of the two liquids, simulations of water and methanol at $300 \mathrm{~K}$ and at supercritical conditions $\left(\mathrm{T}_{\mathrm{H}_{2} \mathrm{O}}=775 \mathrm{~K} ; \mathrm{T}_{\mathrm{MeOH}}=650 \mathrm{~K}\right)$ were performed at constant density (the density corresponds to the one at $300 \mathrm{~K}$ and 1 bar). The simulation parameters were the same as for the other simulations, but the coordinates were written out every $20 \mathrm{fs}$ to resolve the fast dynamics of the hydrogen bonds especially at supercritical conditions. The SPC model was used for water and the OPLS force field for methanol.

In a previous study of van der Spoel et al. [55] an entropic barrier for the breaking of methanol hydrogen bonds at normal conditions was found which was not observed for water. In this work the study of the hydrogen bond dynamics was extended to supercritical temperatures to reveal if the faster dynamics for water also holds for the conditions found at the beginning of the expansion process. The analysis of the dynamics was performed by comparison of the hydrogen bond lifetimes $\tau_{\mathrm{HB}}$ obtained from the integrated autocorrelation function $C_{h}(t)$ as described in detail in Chapter 2.7.1.

At $300 \mathrm{~K}$ the hydrogen bond lifetime in water is four times smaller than the lifetime in methanol $\left(\tau_{\mathrm{HB}, \mathrm{H}_{2} \mathrm{O}}=4.46 \mathrm{ps}, \tau_{\mathrm{HB}, \mathrm{MeOH}}=18.70 \mathrm{ps}\right)$. This result is in agreement with the results of van der Spoel et al. [55]. Under the same density but at supercritical conditions $\left(\mathrm{T}_{\mathrm{H}_{2} \mathrm{O}}=775 \mathrm{~K} ; \mathrm{T}_{\mathrm{MeOH}}=650 \mathrm{~K}\right)$ 
the lifetimes are much smaller, as higher temperatures enhance the dynamics. However, a hydrogen bond in methanol lives two times longer than a hydrogen bond in water $\left(\tau_{\mathrm{HB}, \mathrm{H}_{2} \mathrm{O}}=0.41 \mathrm{ps}, \tau_{\mathrm{HB}, \mathrm{MeOH}}=0.82 \mathrm{ps}\right)$.

This differences in the hydrogen bond lifetimes at supercritical temperatures explains the slower dynamics in the expansion process observed in the experiment and the simulations for methanol compared to water, since the first step in the expansion consists in the formation and dissociation of hydrogen bonds in the supercritical fluid.

\subsubsection{Aggregates}

The comparison of the average density around a molecule (Chapter 3.4.1) and the number of hydrogen bonds per molecule (Chapter 3.4.2) at supercritical conditions leads to a controversial picture. Although the density drops by one order of magnitude, several water molecules are still involved in hydrogen bonds. This controversial picture can be resolved if during the expansion process the liquid was divided in high density and low density regions. As shown in Figure 3.9 the density around a molecule can range from almost zero at the left side to densities comparable with the liquid densities under normal conditions at the right.

To yield a more detailed picture of the density distribution the number of molecules which can be grouped together to an aggregate at a certain time $t$ in the expansion process was analyzed. One molecule belongs to an aggregate when it possesses at least one neighbor of this aggregate which was closer than $3.5 \AA$. For each time step the aggregate size distributions of four to seven trajectories at a certain initial temperature were averaged and aggregates larger than 200 molecules were fitted with a gaussian function. Figure 3.16 displays the number of molecules which belong to a certain aggregate size at a time $t$ during the expansion process of supercritical water. At the beginning of the simulation the density is high and the molecules 

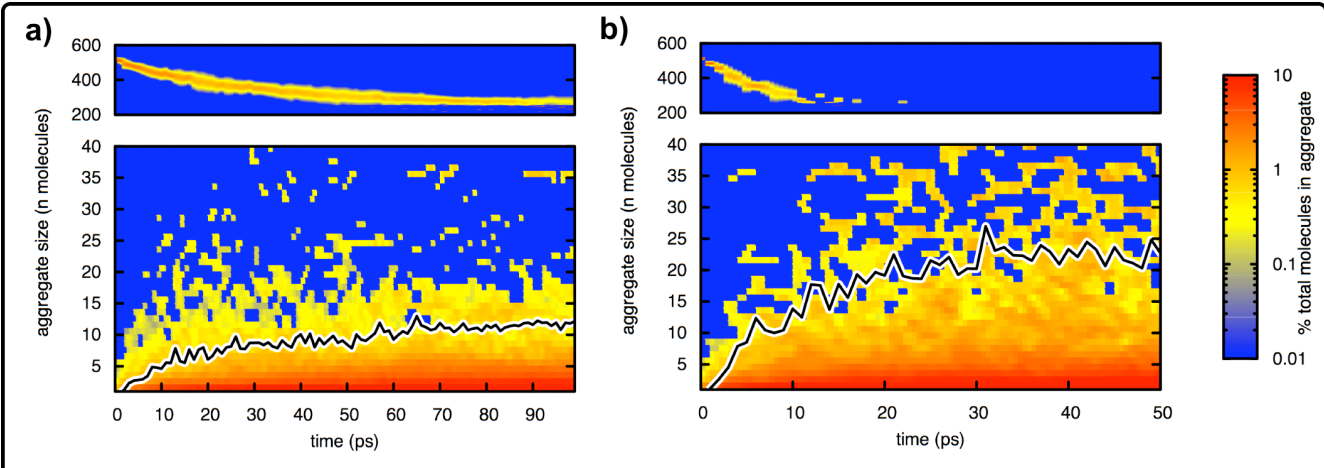

Figure 3.16: Fraction of water molecules belonging to a certain aggregate size at time $t$ during the expansion process of supercritical water. a) Distribution at $775 \mathrm{~K} \mathrm{~b}$ ) Distribution at $1100 \mathrm{~K}$.

form a large aggregate with a size of $\sim 500$ molecules which corresponds to the number of molecules which contribute to the photoelectron spectrum and are in the first nm layer. During the expansion process the large aggregate dissociates into small aggregates. The black line representing the average size of the small aggregates until aggregate sizes of 200 molecules increases during the simulation, which reveals a redistribution from the large aggregate in the beginning to smaller ones with sizes until 10-20 molecules. The number of small aggregates formed during the expansion process is larger for higher temperatures (Figure 3.16b) and not present for the simulations at subcritical conditions.

This small aggregates consisting of 10-20 molecules are assigned to the new band arising in the experiment at supercritical conditions. Photoelectron spectra of protonated water clusters revealed that clusters with sizes between 5 and 20 molecules have ionization potentials of $\sim 11 \mathrm{eV}$ [68], which is the same energy of the new band in the experiment. These studies and the observed simulation results enable the conclusion that during the expansion of supercritical water aggregates are formed. 


\subsubsection{Dynamics of Thermally Excited Water Aggregates: Dimer, Trimer, Tetramer}

Although aggregates are built during the simulated expansion process, they must have long enough lifetimes to be detected by the XUV pulse to correspond to the new band observed in the experiment. On this note, the lifetimes of dimer, trimer and tetramer were studied.

The starting structure of the different aggregates were taken from ab-initio geometry optimization and minimized in the classical force field with the steepest descent algorithm to a convergence of $0.1 \mathrm{~kJ} / \mathrm{mol}$. After minimization, each atom was assigned a random velocity from a Maxwell-Boltzmann distribution at $775 \mathrm{~K}$. Therefore, each atom was given a random velocity from a one dimensional Maxwell-Boltzmann distribution in each direction $(x, y, z)$ and the total kinetic energy of all atoms was calculated. This total kinetic energy with the number of degrees of freedom in the system $(3 N)$ was used to calculate the achieved temperature. The ratio of the achieved and the desired temperature $T$ served as scale factor for all velocities.

For the simulations a time step of $0.1 \mathrm{fs}$ for integration of Newton's equation was used and 101 trajectories with a total length of $100 \mathrm{ps}$ each were simulated. The position of the atoms were written out every 2 femtoseconds. The lifetime of an aggregate was defined as the time after which, one of the $O O$-distance was longer than $4 \AA$ during at least $200 \mathrm{fs}$. It was not distinguished which oxygen atoms were involved, and for the trimer and tetramer the cluster was treated as dissociated already after the first OO-distance exceeded the threshold. Figure 3.17 shows the distribution of lifetimes of the dimer, trimer and tetramer for the trajectories where a dissociation could be observed. The trajectories with no dissociation had a kinetic energy after assignment of random velocity, which was lower than the potential energy of the aggregate. For the dimer (Fig. 3.17a) half of the dissociated aggregates have a lifetime smaller than $2 \mathrm{ps}$. The trimer and 


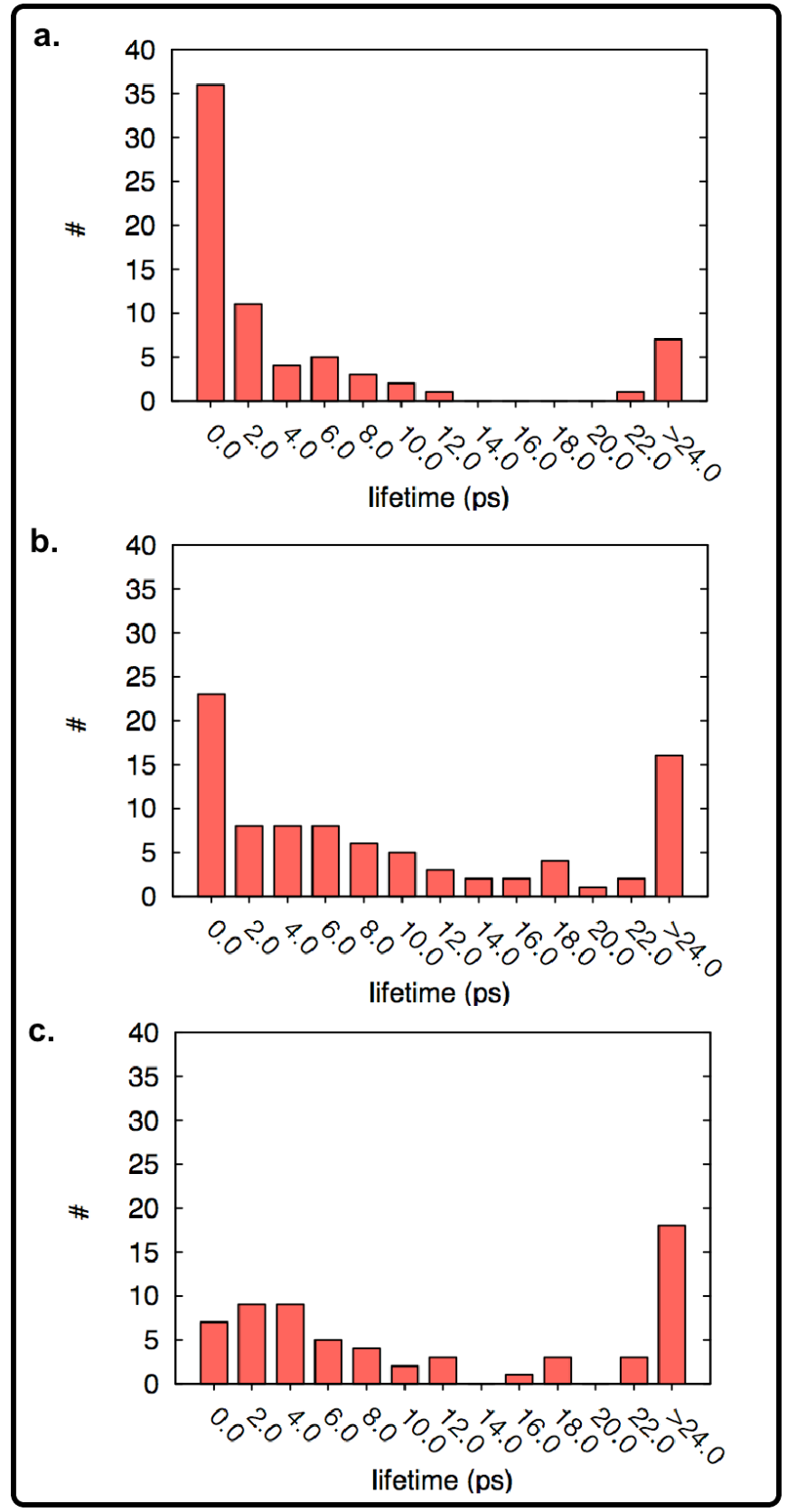

Figure 3.17: Distribution of aggregate lifetimes being in their minimum energy structure at the beginning. a) Dimer (dissociated aggregates 70 from 101) b) Trimer (dissociated aggregates 88 from 101) c) Tetramer (dissociated aggregates 64 from 101). 
tetramer (Fig. 3.17b,c) show distributions of lifetimes which are shifted to longer lifetimes. Only $20 \%$ of the dissociated trimer has a lifetime smaller than 2 ps and only $10 \%$ in the case of the tetramer. The remaining lifetimes of the trimer and the tetramer are distributed over a wide range compared to the dimer and in the case of the tetramer even $\sim 30 \%$ of the trajectories exhibit an aggregate lifetime larger than 24 ps.

This results show that aggregates at supercritical conditions which are in their minimum energy configurations at the beginning have lifetimes which increase with aggregate size and are in the order of picoseconds for the trimer and the tetramer. Although the aggregates in the simulations and the experiment are not built in the minimum energy configurations, the obtained lifetimes may serve as an upper limit. Furthermore, it is expected that larger aggregates consisting of 10-20 molecules encounter even larger lifetimes and enable their ionization with the XUV field.

\subsubsection{Simulated Photoelectron Spectra of Water}

The performed studies using Molecular Dynamics simulations enable a detailed picture of dynamics during the evolution of supercritical metastable water. But still the question remains how the energies of the ground and ionized state of the molecules involved in the ionization process evolve in time. In addition, it is a moot question which state is responsible for the observed shift in the measured photoelectron spectrum. One suitable tool to study these issues is the Quantum Mechanics/Molecular Mechanics (QM/MM) method which allows to study the energy of the ground and exited state of a subset of molecules in an environment of solvent molecules treated at the MM level. This calculation are carried out for different MM configurations at different times during the expansion process to obtain ensemble averages, which are compared with the experimental results (for details on the QM/MM method see Chapter 2.5). 
To obtain ensemble energies for the ionized and ground state of the molecules at every picosecond 100 water molecules and their five nearest neighbors were randomly selected from the molecules contributing to the photoelectron spectrum in the water simulations with $\sim 430000$ molecules and initial temperature of $775 \mathrm{~K}$ (see Fig. 3.9). Calculations with more neighbors (7-9 molecules) on configurations of an equilibration run of liquid water at normal conditions did not reveal any differences in the simulated photoelectron spectra. For that reason only five neighbors were taken into account and for each of the 100 water molecules a QM/MM single point calculation was carried out in which the six mentioned water molecules were treated with the Quantum Mechanics (QM) and the molecules contained in a sphere with radius $1 \mathrm{~nm}$ around the six molecules with Molecular Mechanics (MM). The QM/MM method implemented in the GROMACS-package was used and the MM water molecules were described with the SPC water model. For the QM molecules the MP2 method with the cc-pdvz basis set was chosen (for details see Chapter 2.4.3). This method and the corresponding basis set provide accurate energies for hydrogen bonded systems since they include electron correlation effects. Although more advanced methods would improve the energies, the computational efforts would not allow the computation of enough conformations to yield an accurate ensemble average, which is of more relevance for the studied systems.

The calculation of the energy of the ground state at each configuration yields also a set of orbital energies (see Chapter 2.4.2). These orbital energies are compared to the electron binding energies in the measured photoelectron spectrum within the Frozen Orbital approximation (Koopmans' theorem). On this basis each obtained orbital energy of the six water molecules in the QM region was fitted with a gauss function of the width $0.1 \mathrm{eV}$ (approximated resolution in the experimental setup) and all gauss functions were summed up together and then normalized to yield an area of one. This distribution represents the fraction of electrons with the respective binding energies at a specific time during the expansion process. However, it 


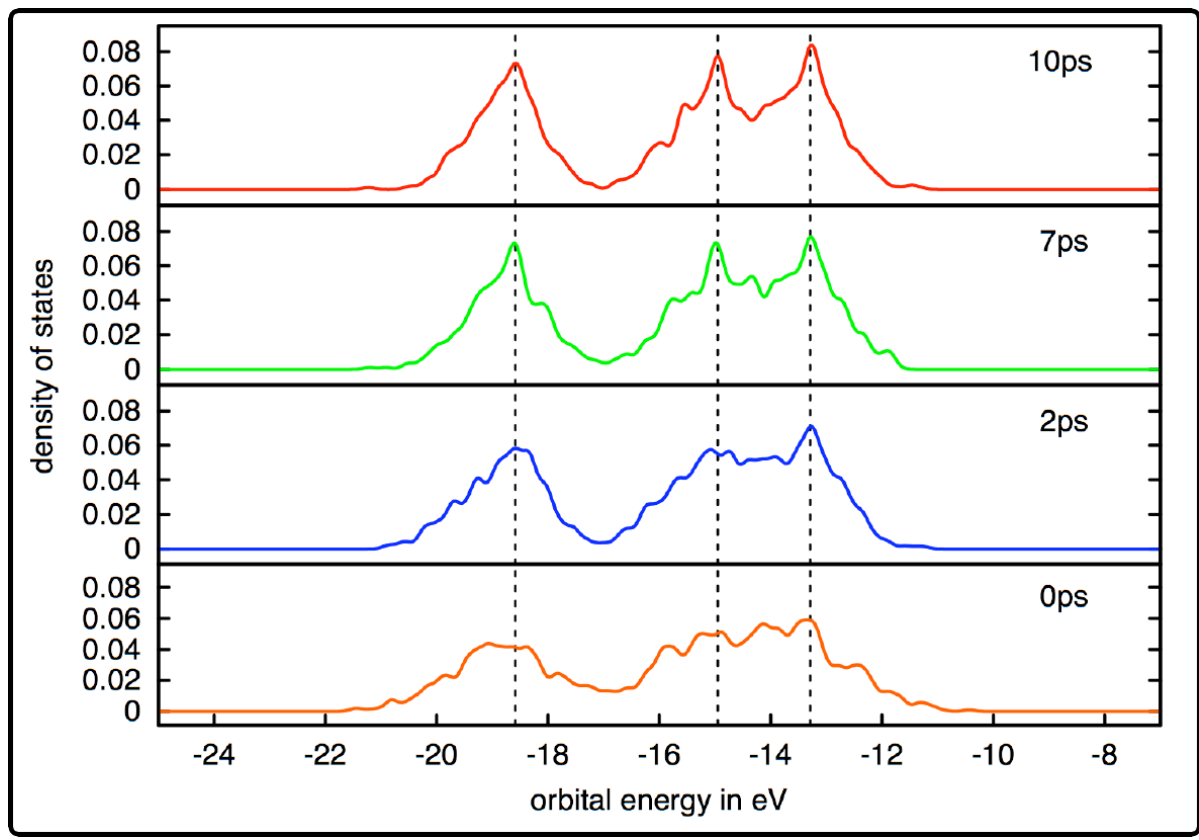

Figure 3.18: Distribution of electron binding energies of six water molecules treated with $\mathrm{QM} / \mathrm{MM}$ for different times during the expansion process. Vertical dashed lines represent the orbital energies of the water monomer in the configuration of the SPC water model.

does not yield information about the probability by which this electron is ejected.

Figure 3.18 displays the distribution of electron binding energies at the beginning and at 2, 7 and $10 \mathrm{ps}$ during the expansion process. The vertical dashed lines represent the orbital energies of the electrons in a water monomer at the configurations given by the SPC water model. This orbital energies differ from the orbital energies of the water monomer at the minimum energy configuration. As can be seen, at the beginning the distribution is wide and ranges until orbital energies of $10.5 \mathrm{eV}$ in accordance with the experimental results. At later times the distribution narrows to the orbital energies of the water monomer. However, the distribution still reveals electrons with energies lower than the highest molecular orbital of 


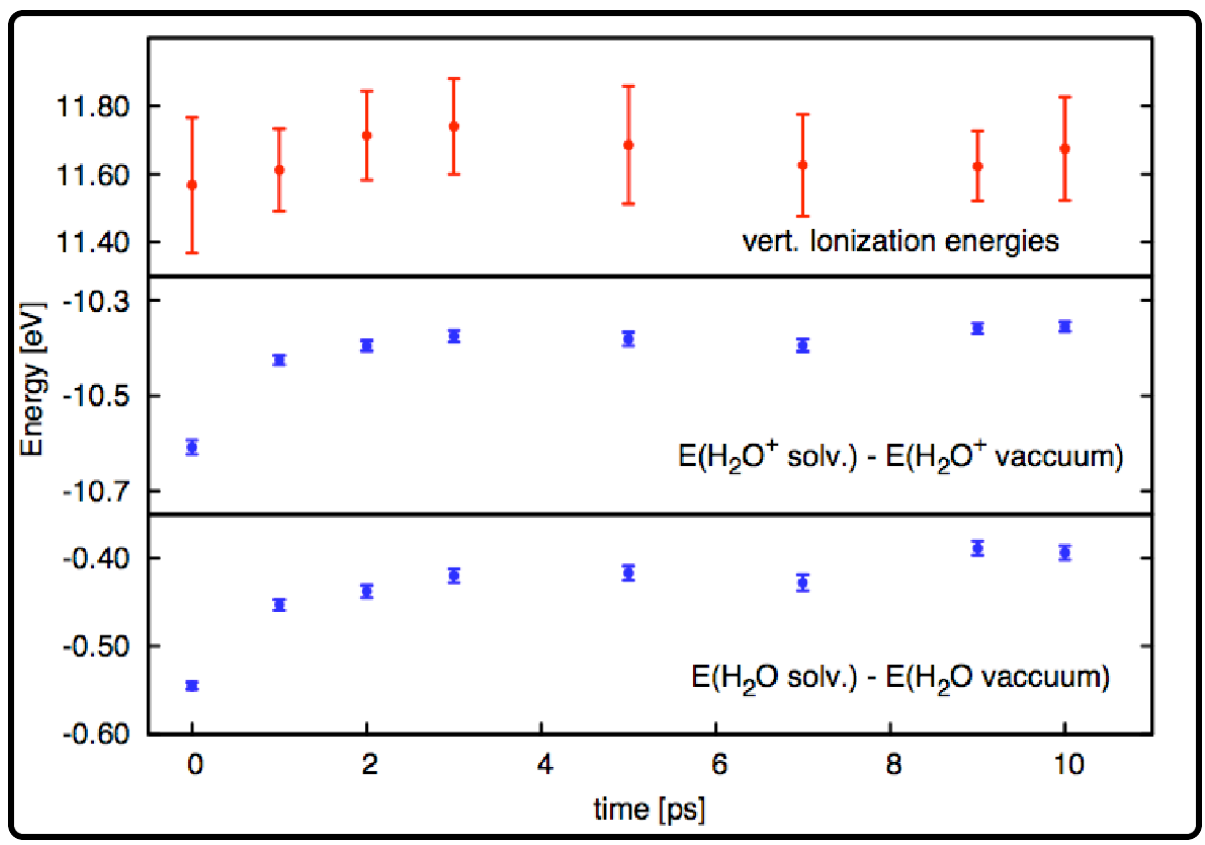

Figure 3.19: Top panel: Average vertical ionization energies of the electrons in the highest occupied molecular orbital of the QM-water molecules. Middle panel: Energy difference between the average energy of a solvated ionized QM water molecule and the energy of an ionized water monomer in vacuum. Lower panel: Energy difference between the average energy of a solvated neutral QM water molecule and the energy of a neutral water monomer in vacuum.

the water monomer. This electrons contribute to the observed liquid band in the measured spectra.

The agreement between the observed shifts in the measured photoelectron spectra and the simulated ones shows that the QM/MM method is able to provide a good representation of the electron binding energies during the process. To distinguish between contributions of the ground and ionized states to the measured shifts of the electrons in the liquid band, the energy after the removal of an electron from the highest occupied molecular orbital (HOMO) of the QM waters was calculated. For each configuration at each time step a new $\mathrm{QM} / \mathrm{MM}$ calculation at the same configuration was done 
in which one electron is taken out of the highest molecular orbital. The obtained total energy of the QM water molecules was divided by the number of molecules yielding an average energy per ionized water molecule. This averaged energy was further averaged over all configurations at time $t$. The energy difference between this energy and the energy of an ionized water monomer in vacuum corresponds to its stabilization due to the surrounding molecules. The same calculation was carried out for the neutral QM water molecules.

The evolution of the respective stabilization energies of the ground and ionized state of a water molecule is shown in Figure 3.19 (middle and lower panel). Both stabilization energies decreases almost equally during the expansion process. The sum of the two energies represents the average vertical ionization energy of an electron in the HOMO orbital of one water molecule and is shown in Figure 3.19 top panel. There is an exponential shift towards the ionization energy of the monomer in vacuum of $12.6 \mathrm{eV}$ at the beginning. This result matches very nicely with measured spectra which reveal a large shift of the liquid band to larger binding energies at the beginning. 


\section{Chapter 4}

\section{Laser Induced Isolation of Charges from Water}

This Chapter deals with the isolation of charges from liquid water. In the following, the experiment used for mass spectrometrical analysis of ions and charged molecules will be described.

Furthermore, computer simulations will provide a microscopic picture of the desorption mechanism and explain the softness of the method in comparison to other techniques. A general mechanism for the creation of charges will be proposed and its predictions will be compared to the simulation results.

\subsection{Experiment}

The experimental setup for the laser induced isolation of charged molecules from liquid water is displayed in Figure 4.1. A mixture of liquid water and the solute of interest is injected in a vacuum chamber $\left(p=5 \cdot 10^{-5} \mathrm{mbar}\right)$ with a HPLC pump through a nozzle with the diameter of $14 \mu \mathrm{m}$ (Figure 3.2). The injected solution yields a liquid beam in vacuum, described in Chapter 3.2 , in which the liquid is at normal conditions. An infrared laser tuned 


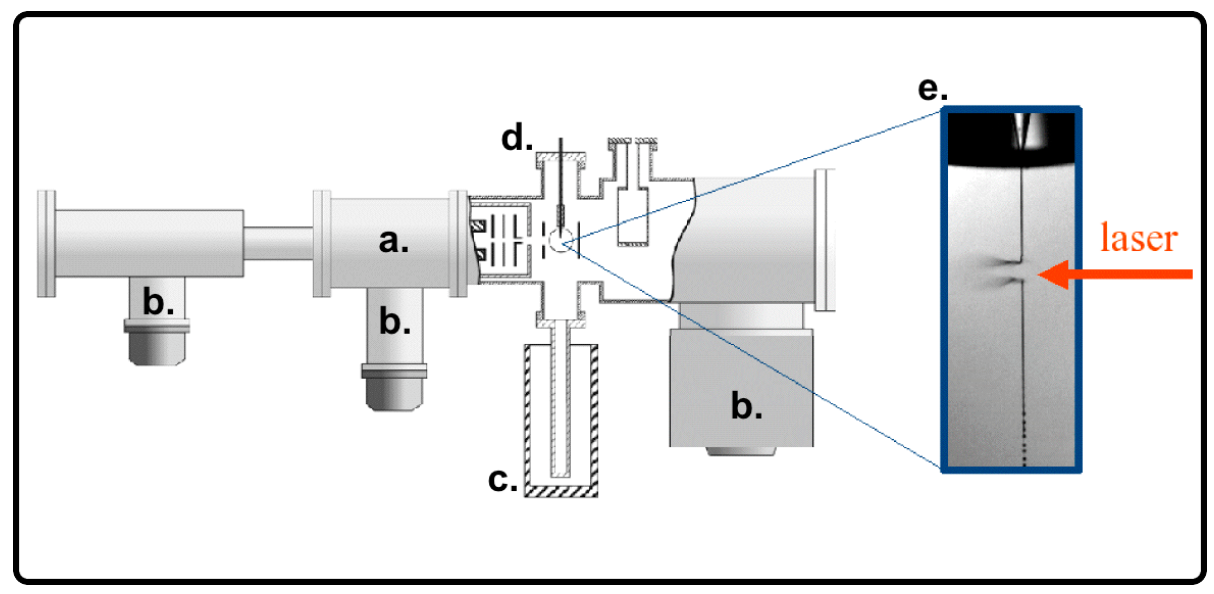

Figure 4.1: Experimental setup for laser induced isolation of charged molecules from liquid water. a.) Mass spectrometer for analysis of charged molecules. b.) Vacuum pumps. c.) Cryo trap for liquid beam. d.) Injection site for mixtures of liquid water and the solute. e.) Zoomed picture of the liquid beam $(10 \mu \mathrm{m})$ after the irradiation with the infrared laser.

at the $\mathrm{OH}$-stretch vibration of water $(\lambda=2650 \mu \mathrm{m})$ with a pulse energy of $0.6 \mu \mathrm{J}$ and a pulse length of $10 \mathrm{~ns}$ is focused on this water filament. At a distance of $10 \mathrm{~mm}$ to the filament a skimmer with a diameter of $1.5 \mathrm{~mm}$ is positioned perpendicular to the liquid and the laser beam. This skimmer samples the isolated charged molecules flying into the Time Of Flight Mass Spectrometer (TOF-MS). At the entrance of the mass spectrometer pulsed ion optics accelerate the positively charged molecules with a voltage of $4-6 \mathrm{kV}$ in the mass spectrometer at a switchable time delay $\Delta t$ after the laser irradiation. This pulsed electric field allows a screening of the charged isolation products after the irradiation with the infrared pulse. For further details on the experimental setup the reader is referred to $[69,21,20]$.

With this experimental setup biomolecules solvated in the liquid beam are transfered into the gas phase and detected by the mass spectrometer. The isolation of biomolecules from the liquid beam is soft, thus, also the characterization of fragile proteins or protein complexes without fragmentation 


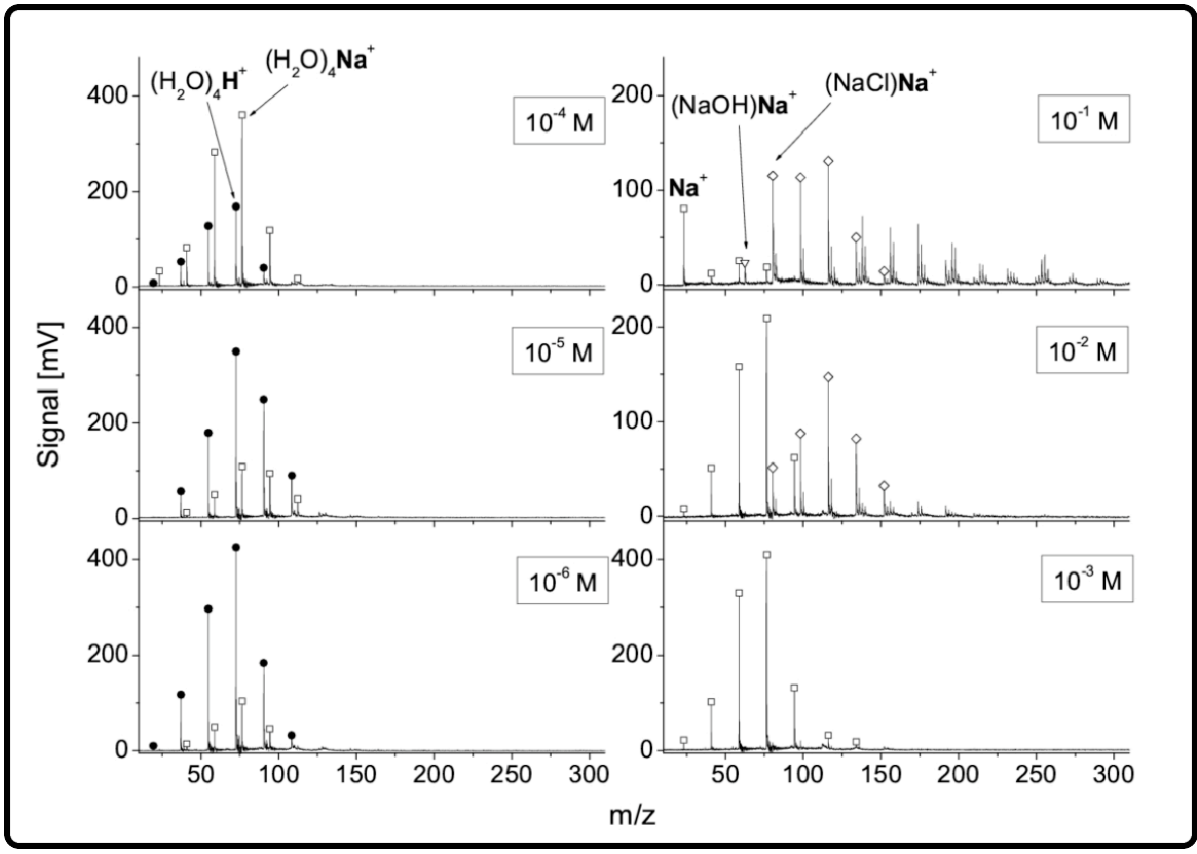

Figure 4.2: Mass spectra of $\mathrm{NaCl}$ solutions at concentrations ranging from $10^{-6}$ to $10^{-1} \mathrm{M}$ from [70].

becomes possible. The softness of the desorption process will be discussed in Chapter 4.3.3.

However, for their detection the desorption process must be accompanied with a generation of charges. To study this creation of charges, NaClsolution with concentrations ranging from $10^{-6}$ to $10^{-1} \mathrm{M}$ were analyzed. Figure 4.2 displays measured mass spectra from $\mathrm{NaCl}$ solutions at different concentrations taken from [70]. At low ion concentrations $\left(10^{-6}-10^{-5} \mathrm{M}\right)$ protonated water aggregates $\left(\left(\mathrm{H}_{2} \mathrm{O}\right)_{n} \mathrm{H}_{3} \mathrm{O}^{+}\right)$beside $\mathrm{Na}^{+}$-water aggregates are observed. At higher ion concentrations $\left(10^{-4}-10^{-3} \mathrm{M}\right)$ the protonated water aggregates diminish and the number of $\mathrm{Na}^{+}$-water aggregates increases. For concentrations larger than $10^{-2} \mathrm{M}$ also aggregates with more than one ion per aggregate are observed. In Chapter 4.3.2 the appearance of the combined aggregates enclosing positive and negative ions and their 
total intensity as a function of the concentration in the liquid will be discussed and the mechanism responsible for the generation of charges upon desorption will be explained.

\subsection{Simulation Setup}

Since an atomistic simulation of the total liquid beam $(\mathrm{d}=14 \mu \mathrm{m})$ is computationally not feasible, rectangular model systems with two short sides of $4 \mathrm{~nm}$ and lengths of 50, 70 and $100 \mathrm{~nm}$ containing between 27078 and 54432 water molecules were simulated with periodic boundary conditions. The simulations were performed with the GROMACS simulation package [33]. The rectangular water boxes were equilibrated at $300 \mathrm{~K}$ and 1 bar using the temperature and pressure coupling algorithm of Berendsen [65]. Water molecules were represented by the SPC water model. To analyze wether the results are sensitive to the force filed parameters represented in different water models, also simulations with the TIP4P model were performed, however, the results obtained were the same. For a detailed description of the water models the reader is referred to Chapter 2.6.1. The time step to integrate Newton's equation was set to $1 \mathrm{fs}$. The short range Coulomb interactions were calculated explicitly up to $1.2 \mathrm{~nm}$ and for the long range interactions the Particle Mesh Ewald method was used [66]. The Van-derWaals interactions were shifted at a distance of $0.8 \mathrm{~nm}$ and then leveled off to 0 at $1.0 \mathrm{~nm}$.

For the simulations at different ion concentrations a corresponding number of sodium and chloride ions was placed at random positions (replacing oxygen atoms of water molecules). Subsequently, a Monte Carlo optimization with respect to the total energy was performed to assure a proper distribution (for details see [71]). After ion placement the simulation box was 
equilibrated further to allow for the relaxation of the water molecules near to the ions.

\subsubsection{Temperature Gradient}

In the experiment the energy introduced with the infrared light on the liquid filament excites the $\mathrm{OH}$-stretch vibration of water molecules. This energy is assumed to be redistributed instantaneously over all degrees of freedom of the liquid. This approximation is valid, as studies reported time scales for this redistribution to be as fast as $\sim 50 \mathrm{fs}$ and, therefore, much shorter than the time scale of the desorption process [2].

Under this assumption the energy deposited through the laser is distributed in the liquid in the same way as the electromagnetic radiation is absorbed. Absorption of electromagnetic radiation in a sample is described by LambertBeer's Law,

$$
I(d)=I_{0} \exp (-\epsilon \cdot c \cdot d)
$$

where $I(d)$ represents the intensity of the radiation at the penetration depth $d$ into the sample, $I_{0}$ is the intensity of the radiation outside the sample, $\epsilon$ is the molar absorption coefficient of the sample (at $300 \mathrm{~K}$ ), and $c$ the concentration. As the distribution of the energy is instantaneous, the laser beam produces an exponential decay of thermal energy in the liquid, represented as a temperature gradient. Using the absorption coefficient of liquid water at $300 \mathrm{~K}$ and its concentration, the penetration depth reaches $\sim 1 \mu \mathrm{m}$ into the liquid beam in the experiment. However, the simulated water boxes were much smaller. To study the effect of the penetration depth on the simulation results, it was varied along with the amounts of deposited energy.

The temperature gradient was introduced by dividing the water box from 

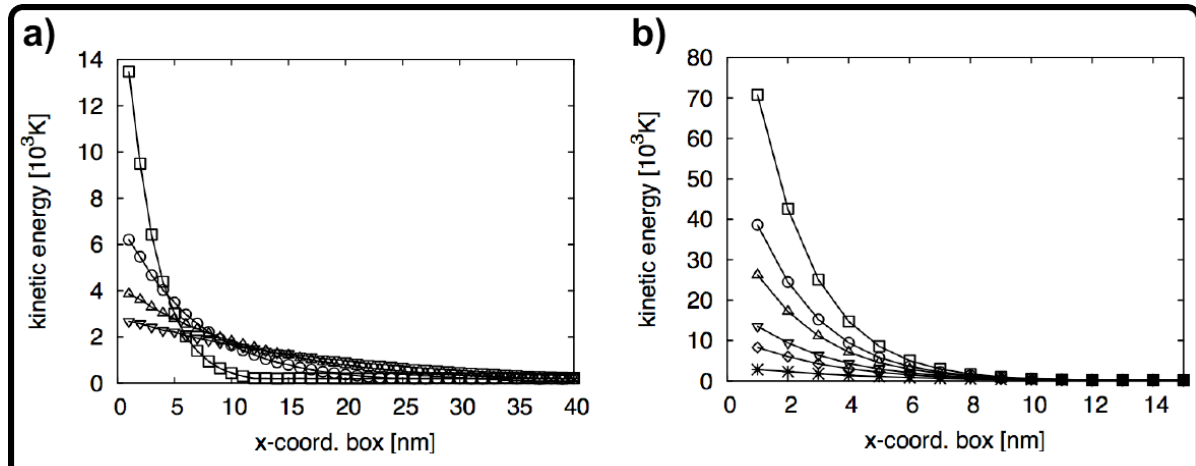

Figure 4.3: Kinetic energy gradients along the long side of the water box ( $\mathrm{x}$ coord.). a) Gradients with deposited energy of $126 \mathrm{~kJ} / \mathrm{mol}$ varying the penetration depth: $\square=12 \mathrm{~nm}, \bigcirc=24 \mathrm{~nm}, \triangle=36 \mathrm{~nm}$ and $\nabla=48 \mathrm{~nm}$. b) Gradients with a penetration depth of $12 \mathrm{~nm}$ and different deposited energies: $\square=527 \mathrm{~kJ} / \mathrm{mol}, \bigcirc=310 \mathrm{~kJ} / \mathrm{mol}, \triangle=222 \mathrm{~kJ} / \mathrm{mol}$, $\nabla=126 \mathrm{~kJ} / \mathrm{mol}, \diamond=84 \mathrm{~kJ} / \mathrm{mol}$ and $*=35 \mathrm{~kJ} / \mathrm{mol}$.

the left into slices of $1 \mathrm{~nm}$ width up to the penetration depth $d$. Each slice was assigned a temperature $T$ according to an exponential function starting with temperature $T_{0}$ at the left side of the water box and reaching $300 \mathrm{~K}$ at the penetration depth $d$. All atoms within each slice at temperature $T$ was assigned a random velocity from a Maxwell-Boltzmann distribution: Each atom was first given a random velocity from a one dimensional Maxwell distribution at the corresponding temperature in each direction $(x, y, z)$ and the total kinetic energy of all atoms was calculated. This total kinetic energy was used with the number of degrees of freedom in the system $(3 N)$ to calculate the achieved temperature. The ratio of the achieved and the desired temperature $T$ served as scale factor for all velocities.

The total deposited energy into the simulation box equals the difference between the kinetic energy introduced by the gradient and the kinetic energy of the corresponding atoms at $300 \mathrm{~K}$.

Figure 4.3a displays the gradient of the resulting kinetic energy for different penetration depths and a total deposited energy of $126 \mathrm{~kJ} / \mathrm{mol}$. Figure $4.3 \mathrm{~b}$ shows the gradients for a constant penetration depth of $12 \mathrm{~nm}$ but different 


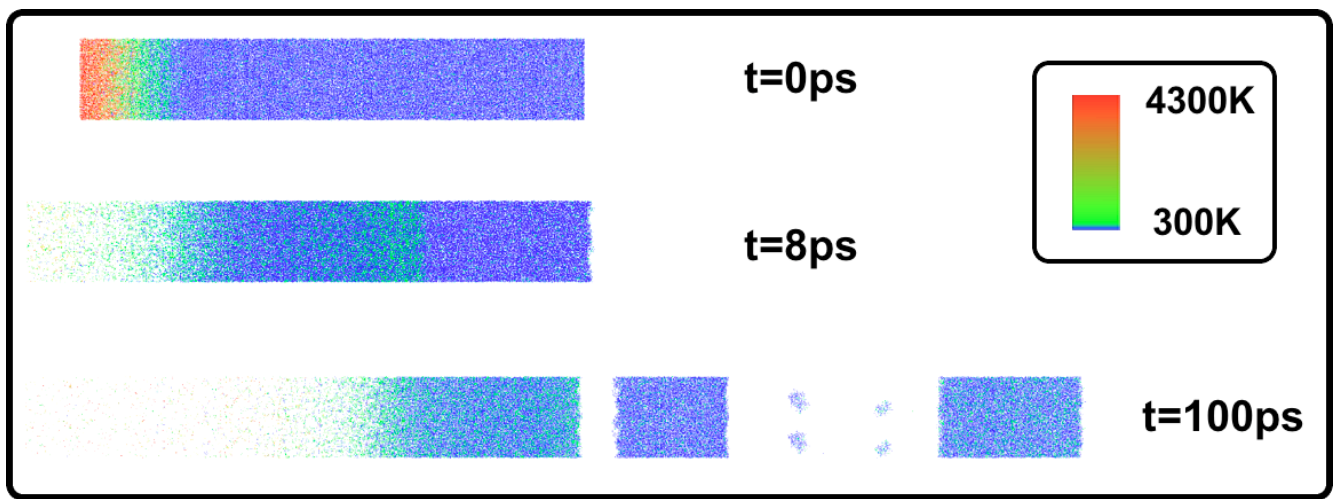

Figure 4.4: Snapshots of the simulated desorption process in liquid water with a water box of $50 \mathrm{~nm}$ length, penetration depth of $12 \mathrm{~nm}$ and deposited energy of $222 \mathrm{~kJ} / \mathrm{mol}$ (water molecules are colored according to their kinetic energy. Two periodic boxes per snapshot are shown).

deposited energies ranging from 527 to $35 \mathrm{~kJ} / \mathrm{mol}$. The different penetration depths and deposited energies represent experimental conditions where a change to longer wavelengths reduces the penetration depth or others where larger laser intensities at the same wavelengths enlarge the amount of energy deposited in the liquid filament.

After the temperature gradient was applied, the simulation box was extended to $300 \mathrm{~nm}$ and the water box was centered in the middle. A layer of argon atoms at the left side of the box served as repellent for molecules coming from the left hot part of the liquid to reenter the box at the right side because of periodic boundary conditions. The argon atoms had a distance of $0.1 \AA$ and were kept at fixed position during the simulation. This new simulation box was simulated at constant volume and energy for $100 \mathrm{ps}$ with the mentioned parameters. 


\subsection{Results}

Figure 4.4 displays snapshots of the simulation with a water box of $50 \mathrm{~nm}$ length, a deposited energy of $222 \mathrm{~kJ} / \mathrm{mol}$ and a penetration depth of $12 \mathrm{~nm}$. Each molecule is assigned a color according to its kinetic energy. At the beginning ( $t=0 \mathrm{ps}$ ) the temperature gradient at the left side of the water box is represented by a color gradient from red to blue. After 8 ps, part of the heated molecules have evaporated into vacuum and in the middle of the box a region representing high kinetic energy is observed. This high kinetic energy region locates the front of a shock wave which travels through the water box during the first $20 \mathrm{ps}$. The properties of this shock wave will be the topic of the following chapter. At the end of the simulation ( $t=100 \mathrm{ps}$ ) the box is split into two large layers and two small droplets. Although the snapshots are the result of one simulation only, large number of performed simulation under equal conditions revealed the same results. The properties of these ablated layers as a function of deposited energy and penetration depth will be discussed in the following Chapter.

\subsubsection{Shock Waves}

As already noted in the previous chapter the introduced temperature gradient forms a shock wave traveling through the water box.

In order to characterize the shock wave, each picosecond the mass density across the long side of the simulation box was calculated. Figure 4.5 displays this mass density along the long side of the simulation box, as a color gradient at different times during the desorption process. At $t=0$ ps the water box with the length of $50 \mathrm{~nm}$ is in the middle of the elongated simulation box (x-coord $=150-200 \mathrm{~nm}$ ) and its density corresponds to the liquid density of the SPC water model at $300 \mathrm{~K}$ and 1 bar $(\rho=976 \mathrm{~g} / \mathrm{l})$. In the first 20 ps a high density region (red color) travels from the left side of the wa- 


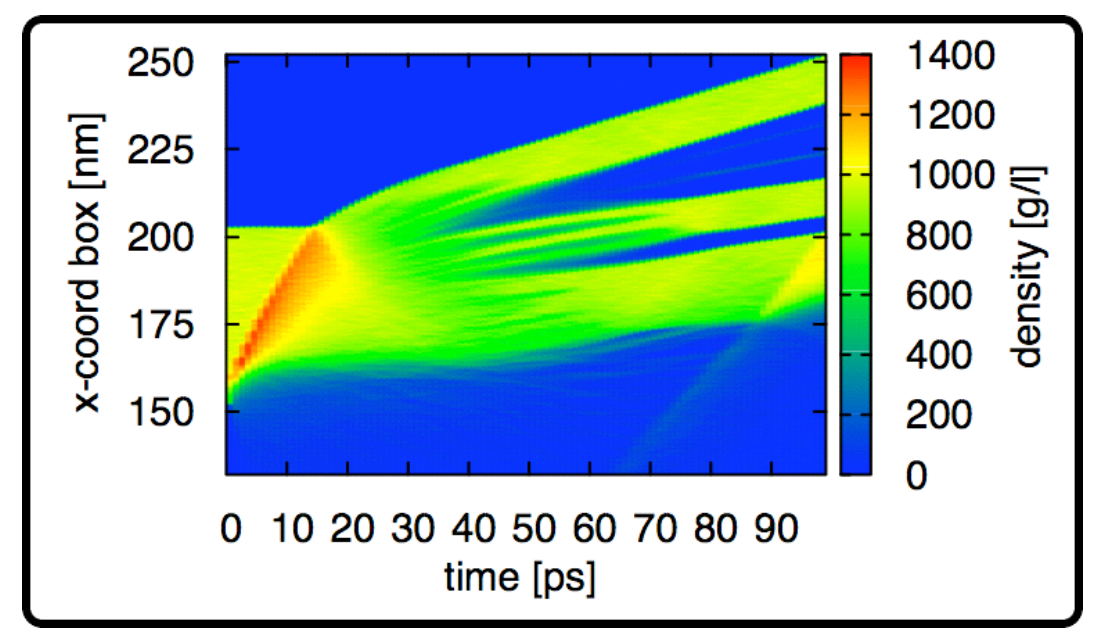

Figure 4.5: Density profile during the desorption process along the box for the simulation with a penetration depth of $12 \mathrm{~nm}$ and deposited energy of $74 \mathrm{~kJ} / \mathrm{mol}$.

ter box $(\mathrm{x}$-coord $=150 \mathrm{~nm})$ to the right side $(\mathrm{x}$-coord $=200 \mathrm{~nm})$ where it hit the liquid vacuum interface. The steepness of a line connecting this high density regions yields a velocity $u_{s}$ of $3.57 \mathrm{~km} / \mathrm{s}$. This velocity, at which the disturbance is propagated in the liquid, is larger than the velocity of sound in liquid water $\left(v_{\text {sound, } \mathrm{H}_{2} \mathrm{O}}=1.46 \mathrm{~km} / \mathrm{s}\right.$ [72]) and corresponds to observed propagation velocities of shock waves ranging from 3 to $8 \mathrm{~km} / \mathrm{s}$ [73].

To compare experimental shock waves to the ones obtained during the simulation some basic properties have to be introduced. The behavior of shock waves is governed by the Rankine-Hugoniot equations. These equations result from the consideration of a one-dimensional steady flow of a fluid at the shock front, where conservation of mass (eq. 4.2), momentum (eq. 4.3) and energy (eq. 4.4) is required.

$$
\begin{aligned}
\rho_{1} u_{1} & =\rho_{2} u_{2} \\
p_{1}+\rho_{1} u_{1} & =p_{2}+\rho_{2} u_{2} \\
e_{1}+\frac{p_{1}}{\rho_{1}}+\frac{u_{1}^{2}}{2} & =e_{2}+\frac{p_{2}}{\rho_{2}}+\frac{u_{2}^{2}}{2}
\end{aligned}
$$


The variable $\rho$ represents the density, $u$ the flow velocity of the particles, $p$ the pressure and $e$ the internal energy. The indices 1 and 2 refer to properties before and after the shock front. Denoting the velocity of the shock wave as $u_{s}$ the flow velocity of the particles after the shock front $u_{2}$ equals $\left(u_{s}-u_{1}\right)$. Rice and Walsh [73] measured the flow velocity $u_{2}$ in water for different shock wave velocities and reported a value of $0.952 \mathrm{~km} / \mathrm{s}$ for a shock wave velocity of $3.354 \mathrm{~km} / \mathrm{s}$. To compare the measured value of $u_{2}$ with the simulations, the velocity of the center of mass of all molecules which are less than $1 \mathrm{~nm}$ ahead from the shock front for a given snapshot was calculated (the shock front was defined at the jump in the density profile). These velocities were averaged over snapshots taken every $2 \mathrm{ps}$ in the time interval between 2 and $10 \mathrm{ps}$. The obtained value for $u_{2}$ was $0.63( \pm 0.04) \mathrm{km} / \mathrm{s}$ at a shock wave velocity $u_{s}$ of $3.57 \mathrm{~km} / \mathrm{s}$ which is in agreement with the measured data.

Having shown that the shock waves in the simulation have comparable properties to measured ones, its effect on the simulation system is analyzed.

After the shock wave traveled through the whole water box it hit the watervacuum interface where it was reflected and looses energy. This lost energy lowers the density maximum of the reflected wave after $20 \mathrm{ps}$. Part of this energy is used to push the former liquid-vacuum interface in the propagation direction of the shock wave. At $t=40 \mathrm{ps}$ the first layer was ablated. The center of mass of this layer moved with a velocity of $0.52 \mathrm{~km} / \mathrm{s}$ in the direction of the shock wave and its length constitutes $14 \mathrm{~nm}$ at the end of the ablation. The corresponding translational kinetic energy represents only $\sim 1.25 \%$ of the deposited energy through the temperature gradient, because the mayor part of the deposited energy is lost by the evaporation of hot molecules to the right. These gas phase molecules built a shock wave, which was reflected at the argon wall. The reflected shock wave can be observed in Figure 4.5 at 90 ps, when it passed into the remaining liquid phase (thin yellow line at $\mathrm{x}$-coord. $=<175 \mathrm{~nm}$ ).

To elucidate the influence of the parameters used in the temperature gra- 


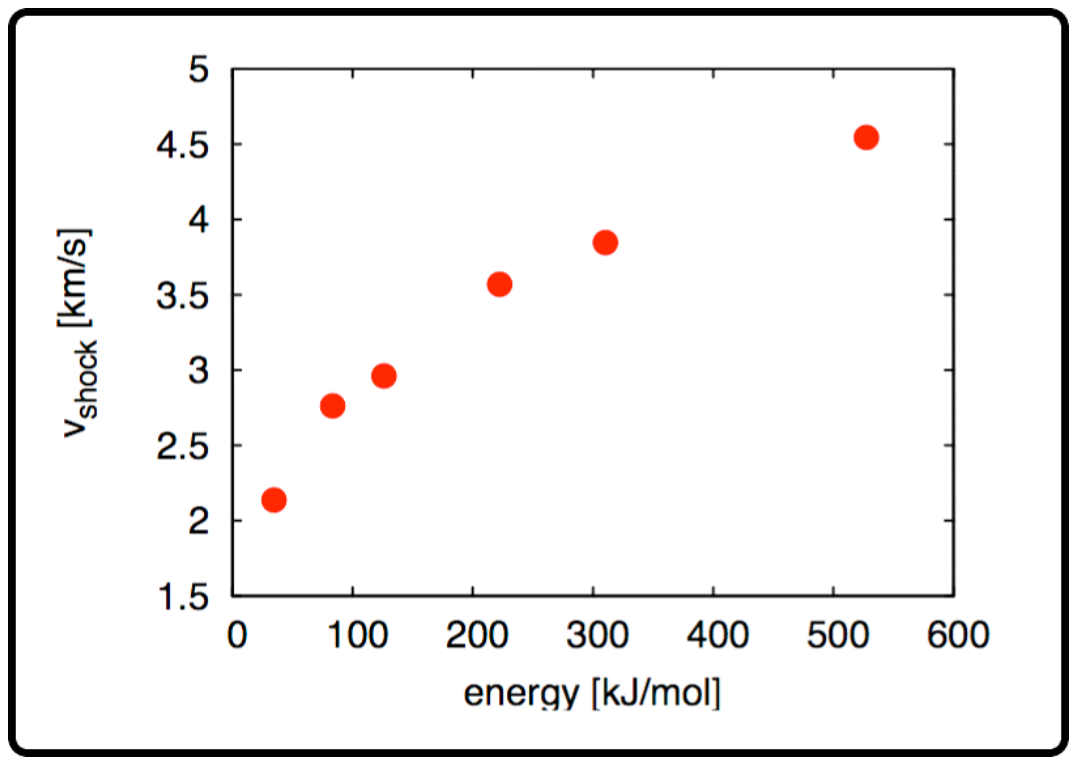

Figure 4.6: Shock wave velocities for different deposited energies, a penetration depth of $12 \mathrm{~nm}$ and a water box length of $50 \mathrm{~nm}$.

dient on the properties of the shock wave and the ablated layers, the same analysis was also performed for simulations with different deposited energies but same penetration depths and length of the water box. Figure 4.6 displays the dependence of the shock wave velocity on the deposited energy for a constant penetration depth of $12 \mathrm{~nm}$ and a water box length of $50 \mathrm{~nm}$. The shock wave velocity increases with the deposited energy until values of $4.5 \mathrm{~km} / \mathrm{s}$, however, only simulations with deposited energies larger than $100 \mathrm{~kJ} / \mathrm{mol}$ showed an ablation of layers at all. In this simulations the center of mass velocities of the first ablated layers increased from $0.3 \mathrm{~km} / \mathrm{s}(126 \mathrm{~kJ} / \mathrm{mol})$ to $1 \mathrm{~km} / \mathrm{s}(527 \mathrm{~kJ} / \mathrm{mol})$ whereas the length of the layer remained constant. Higher center of mass velocities until $3 \mathrm{~km} / \mathrm{s}$ were achieved with simulations where the shock wave was initiated by giving all molecules in the first left $10 \mathrm{~nm}$ of the water box a velocity of $5.4 \mathrm{~km} / \mathrm{s}$ to the left. The shock wave velocity reached was $5.7 \mathrm{~km} / \mathrm{s}$, but the ablation products resemble more small water aggregates than complete layers. 
In addition, the dependence of the penetration depth and the length of the water box on the shock wave was analyzed. Simulations at $126 \mathrm{~kJ} / \mathrm{mol}$ with penetration depths ranging from 12 to $48 \mathrm{~nm}$ in a $70 \mathrm{~nm}$ box exhibited a small increase of the shock wave velocity with the penetration depth up to $24 \mathrm{~nm}$ where it reached a constant value of $2.57 \mathrm{~km} / \mathrm{s}$ - the same as for the water box with $50 \mathrm{~nm}$ length. Increasing the length of the water box at constant penetration depth $(12 \mathrm{~nm})$ and constant deposited energy $(126 \mathrm{~kJ} / \mathrm{mol})$ revealed a decrease in the velocity of the shock wave. The explanation for this finding is that during the propagation of the shock wave through the liquid, the shock wave looses energy because of dissipative effects and therefore, longer water boxes or shorter penetration depths at a given box length lower the shock wave velocities.

\subsubsection{Ion Isolation through Ablation}

The laser irradiation on the liquid beam used in the experiment induces a temperature gradient in the liquid and as shown in the previous Chapter this temperature gradient evokes shock waves in the simulations which disperse the liquid into layers and small droplets. In simulations performed with sodium and chloride ions at two concentrations, 15 and $154 \mathrm{mM}$, some layers carried a net charge, arising from an unequal number of positive and negative ions (details on the simulation setup and parameters are described in detail in Chapter 4.2).

To perform a statistical analysis on the probability of obtaining layers with a net charge $q, 414$ simulations for the lower and 597 for the higher concentration were carried out, each with a total length of 100 ps. All trajectories from one concentration were analyzed with an analysis tool written in a cooperative work with Frank Wiederschein [71]. The formation of an ablated layer and its gap to the remaining liquid was identified through a drop of density along the long side of the box to half of its initial value. In the 


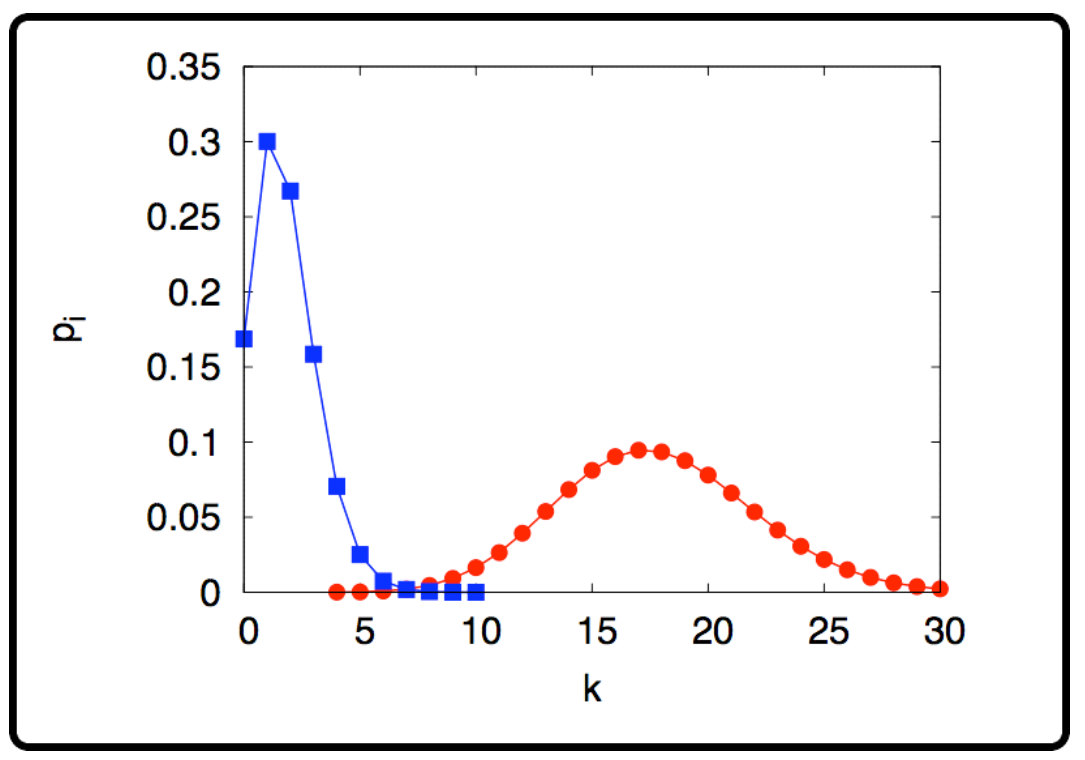

Figure 4.7: Poisson distribution of the number of ions for $\lambda=1.78$ ( $\square$ ) and 17.8 (०) corresponding to an ablated layer of $12 \mathrm{~nm}$ length $\left(\mathrm{V}=192 \mathrm{~nm}^{3}\right)$ at a concentration of 15 and $154 \mathrm{mM}$.

beginning of the simulation several layers of different lengths were observed which merge together to a new layer at later times. Therefore, at the end of the simulation the layer which was at the most right position was taken as reference. Every picosecond during the simulation the length of the last layer was compared to the length of this reference layer and if the deviation was larger than $20 \%$ the following layer to the left and the enclosing gap was merged together with the last layer to a new layer. For each of these layers the number of positive and negative ions were recorded for further analysis.

The difference in these numbers a the end of the simulation constitutes the charge of the respective layer.

Assuming that the probability to find a specific number of positive or negative ions $k$ in the layer is not dependent on the counterions, the distribution of the possible numbers of respective ion $k$ in the layer is given by the Pois- 
son distribution

$$
P(\lambda, k)=\frac{\lambda^{k} e^{-\lambda}}{k !} .
$$

The variable $\lambda\left(\lambda=c \cdot V \cdot N_{\mathrm{A}}\right)$ represents the average number of ions found in a layer of volume $V$ at a given concentration $c$. Figure 4.7 displays the Poisson distribution for possible numbers of ions $k$ in an ablated layer with the length of $12 \mathrm{~nm}\left(\mathrm{~V}=192 \mathrm{~nm}^{3}\right)$ at the concentration of $15(\lambda=1.78)$ and $154 \mathrm{mM}(\lambda=17.8)$. Since the variance of a Poisson distribution equals $\lambda$, the probability for other numbers of ions as the average number increases at larger concentrations.

Under the assumption of independent distributions for positive and negative ions also a probability to find a certain combination of $k$ negative ions and $i$ positive ions can be calculated from the product of the respective probabilities

$$
P(\lambda, k, i)=\frac{\lambda^{i+k} \exp (-2 \lambda)}{i ! \cdot k !}
$$

The probability of obtaining a layer with a charge $\Delta q$ is given as the sum over all probabilities of all possible combination of positive and negative ions with such a total charge

$$
P(\lambda, \Delta q)=\sum_{i=1}^{\infty} \sum_{k=1}^{\infty} \frac{\lambda^{i+k} \exp (-2 \lambda)}{i ! \cdot k !} \delta(\Delta q+k+i) .
$$

Figure 4.8 displays the distribution of charged layers at the end of the simulation for the low and high concentration in comparison to the probability calculated with equation 4.7. For the calculation, $\lambda$ was obtained from the volume of the ablated layers using the average length of all trajectories (11.5 and $12.2 \mathrm{~nm}$ for 15 and $154 \mathrm{mM}$ ) and the specific concentration. The distribution obtained from the simulation at low ion concentration 


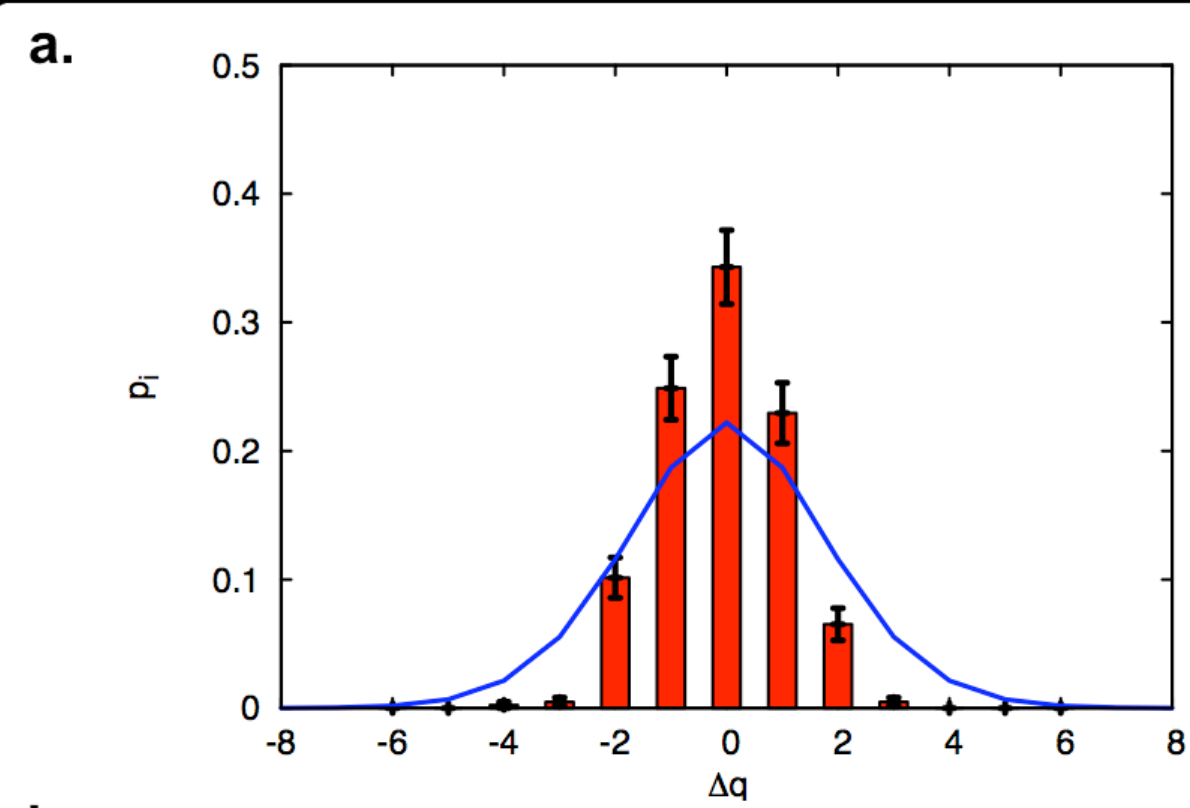

b.

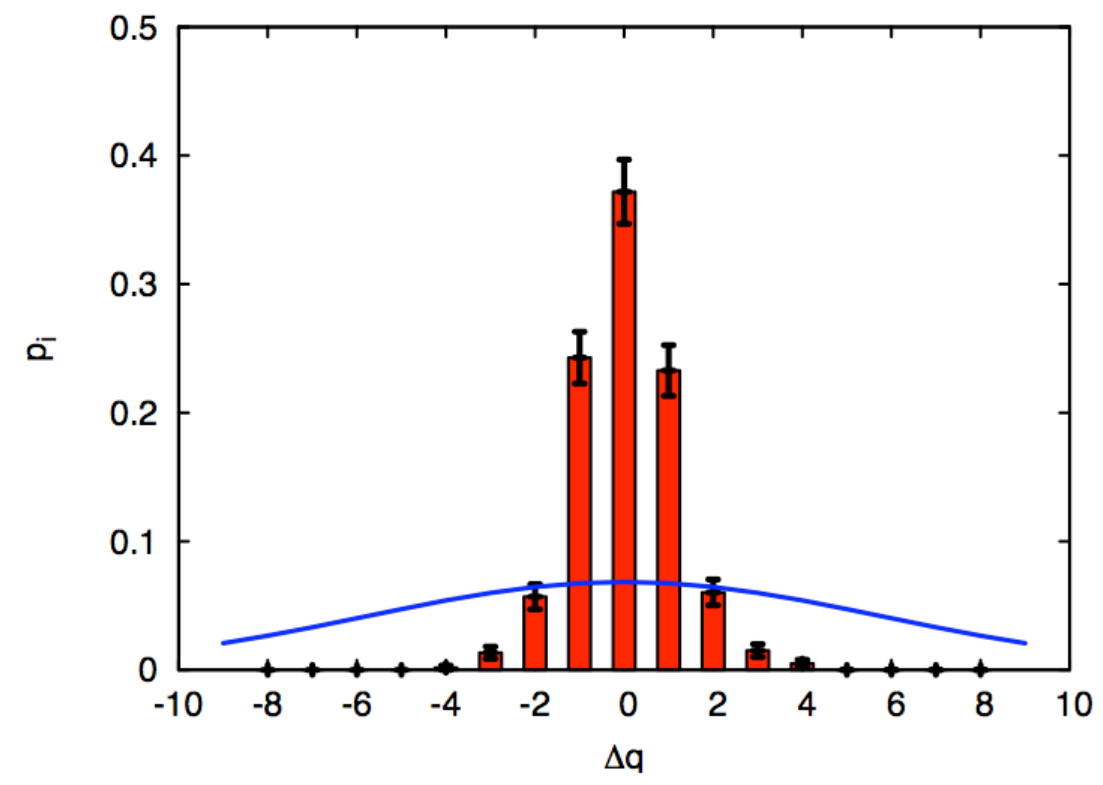

Figure 4.8: Relative occurrence of layers at the end with charge $\Delta q$ from the simulations (red) and the predicted probability by a Poisson distribution of the ions (blue). a.) At a concentration of $15 \mathrm{mM}$ with an average length of the ablated layer of $11.5 \mathrm{~nm}$. b.) At a concentration of $154 \mathrm{mM}$ and an average length of the ablated layer of $12.2 \mathrm{~nm}$. 
(Fig. 4.8a) reveals small deviations from the probability predicted from a Poisson distribution. These deviations become much more significant for $154 \mathrm{mM}$, where the distribution is much narrower exhibiting no layers with a charge larger than five.

Since the predicted probabilities differ from the simulated ones, the assumption of independent positive and negative ion distributions has to be reconsidered. The simulation results suggested that the deviation increases with the concentration. To this end, Monte Carlo simulation in collaboration with Frank Wiederschein were performed, to study until which concentration the predicted matches the simulated probability: Ions were placed first at random positions in a box. The dimension of the box was chosen such that each side equals the respective dimension of the ablated layer $(12.9 x 4 x 4 \mathrm{~nm})$ plus three times the Debye length on each side. The total energy was obtained by summing up the Coulomb interaction of all ions employing the dielectric constant of water $\left(\epsilon_{R}=80\right)$. Thereafter, one ion was placed at a new random position and the total energy was recalculated. The new position was accepted if the total energy decreased or if the Boltzmann weight $\left(\exp \left(-\frac{\Delta E}{k T}\right)\right)$ of the energy difference to the energy before replacement was smaller than a random number between zero and one. For a detailed description on the simulation setup the reader is referred to [71]. After the equilibration process $\left(\frac{\Delta E}{E_{\mathrm{tot}}}<10^{-8}\right)$ the charge of layers with the dimensions of $12.9 \times 4 x 4 \mathrm{~nm}$ in the middle of the box were obtained from different configurations and their distribution was compared to the predicted one by the Poisson distribution. Figure 4.9 displays the respective distributions for different concentrations. It can be observed that for concentrations smaller than $15 \mathrm{mM}$ no differences between the Monte Carlo and the Poisson distribution are present. There the assumption of independent ion distribution is valid. For higher concentrations, however, the interaction of the ions between each other leads to a deviation from the Poisson distribution, which increases with concentration and becomes predominant at $154 \mathrm{mM}$. Since the Monte Carlo simulation was also used to place the ions in the wa- 


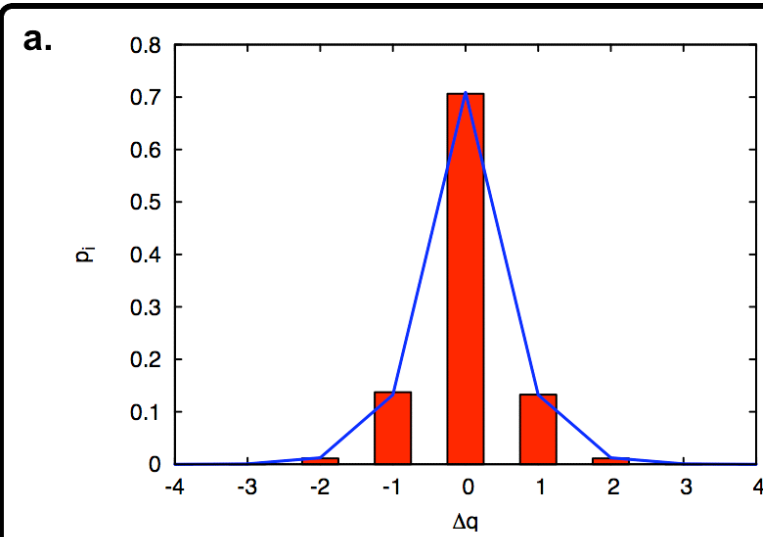

b.

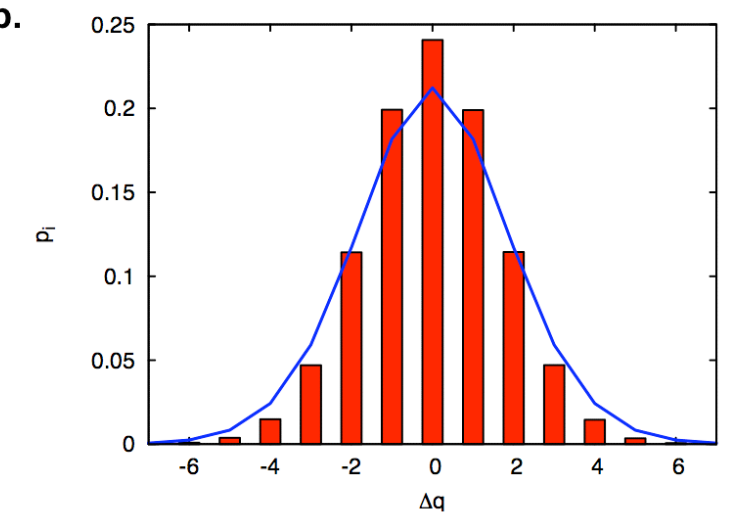

c.

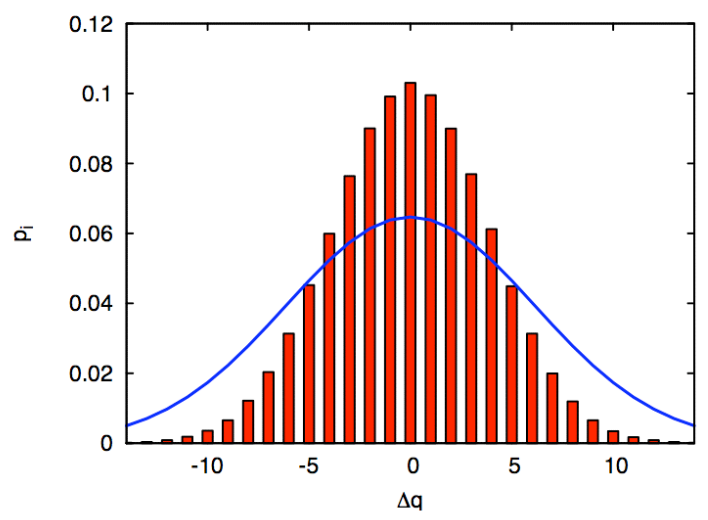

Figure 4.9: Relative occurrence of charged layers obtained by cutting layers with dimensions $12.9 \times 4 x 4 \mathrm{~nm}$ from the middle of a huge box with ions placed by a Monte Carlo simulation (red) in comparison with the probability predicted by Poisson distribution (blue) for different concentrations (a. conc $=1.5 \mathrm{mM}, \mathrm{b} . \quad$ conc $=15 \mathrm{mM}$ and $\mathrm{c}$. conc $=154 \mathrm{mM})$. 


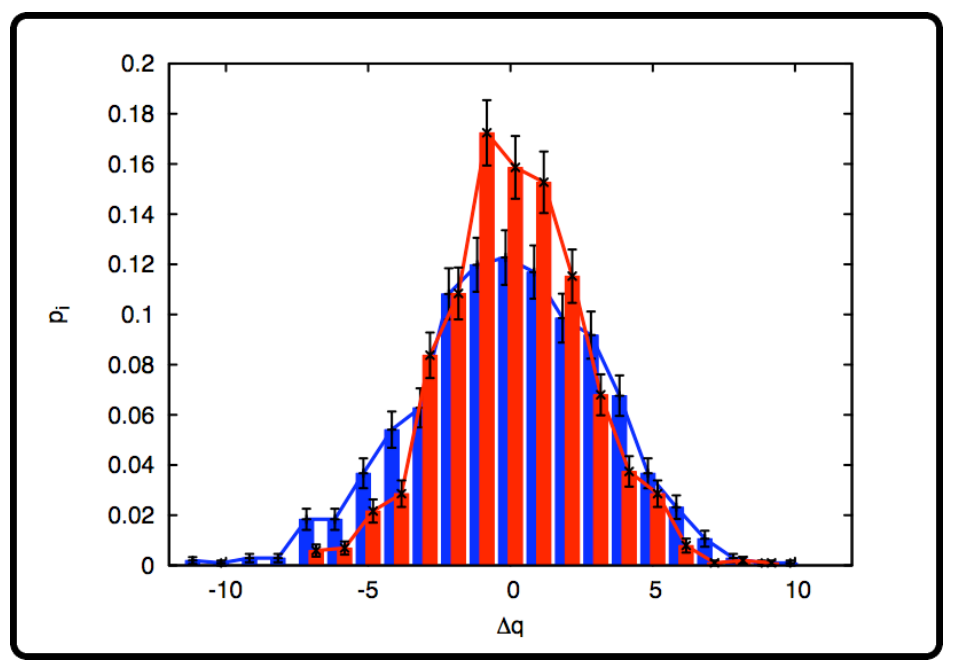

Figure 4.10: Comparison of the distribution of charged layers after ion placement with Monte Carlo in huge box and cutting out water box (blue) and after equilibration of water box with periodic boundary conditions (red).

ter box before applying the temperature gradient, its distribution of charged layers should match the distribution at the beginning of the ablation process if no other process changed the distribution before the ablation. However, comparison of the two distributions revealed differences (see Fig. 4.10). These differences could be assigned to the periodic boundary conditions employed in the equilibration process and during the ablation. Figure 4.10 displays the distribution of charged layers obtained by cutting layers, with the same average dimensions as found in the ablation process, out from the middle of 1000 water boxes with dimensions $60 x 12 x 12 \mathrm{~nm}$ at an ion concentration of $154 \mathrm{mM}$ versus the distribution after the equilibration of the same water box at $300 \mathrm{~K}$ for $40 \mathrm{ps}$ with periodic boundary conditions. As can be observed periodic boundary conditions narrow the distribution.

Changes during the ablation process were studied, by comparison of the distribution of charged layers at the beginning and at the end of the ablation process. Figure 4.11 displays the occurrence of layers with a charge $\Delta q$ at the end in comparison to the distribution at beginning of the ablation 
a.
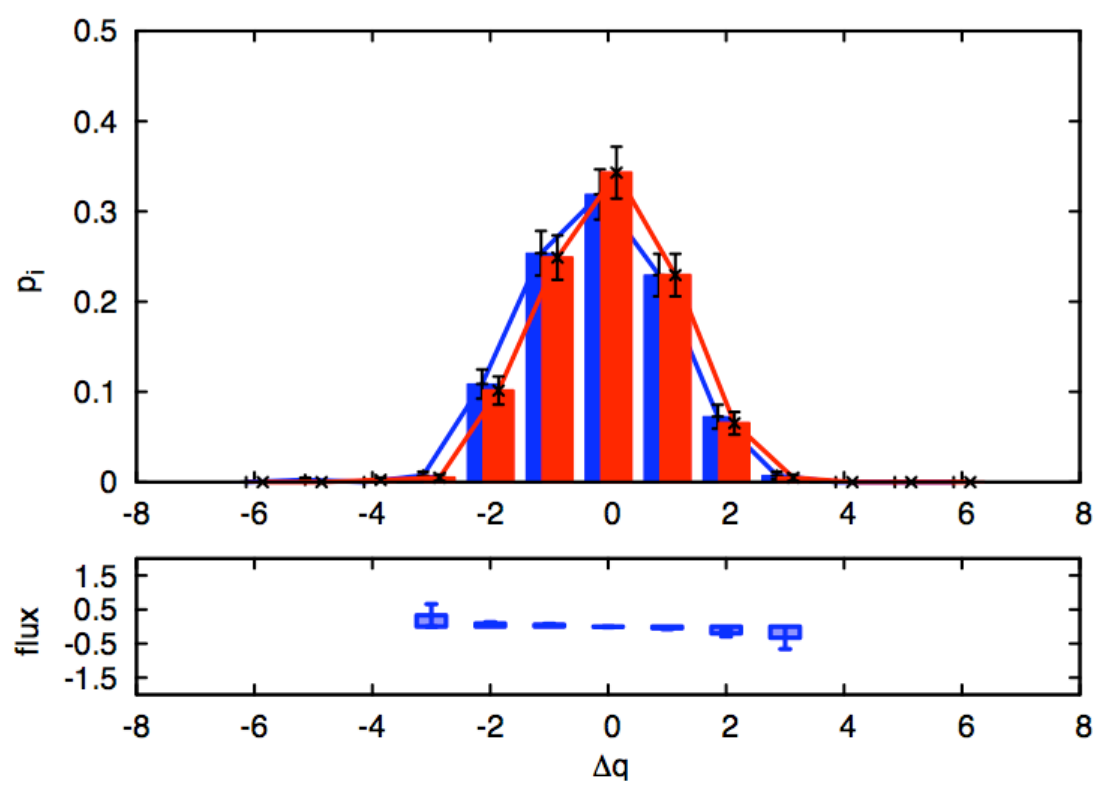

b.
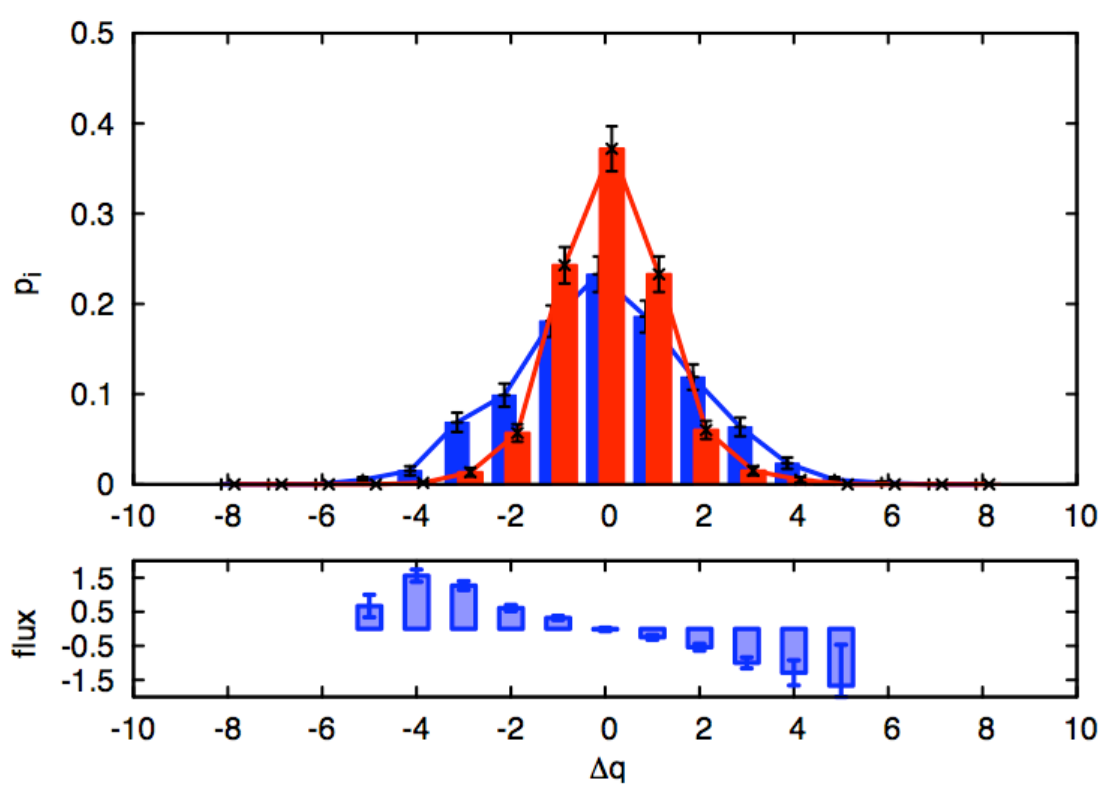

Figure 4.11: Changes in the distribution of charges layers at 15 (a) and $154 \mathrm{mM}(\mathrm{b})$ : Upper panel: Relative occurrence of layers with charge $\Delta q$ at the beginning (blue) and at the end (red) of the simulations. Lower panel: Positive ion flux for trajectories with layers of charge $\Delta q$ at the beginning. 
process. At the smaller ion concentration the distributions at the beginning and at the end are the same. The distribution at $154 \mathrm{mM}$, however, changes significantly: Highly charged layers become less probable and neutral and low charged layers are observed more often at the end than at the beginning.

One possible explanation for this observed deviation is a flux of ions in the gap between the layers and the remaining liquid, which neutralized the former highly charged layers during the ablation process.

In order to evaluate the possible exchange of ions between the layer and the remaining liquid, the position of the ions in the gap, as well as the position of the gap in the simulation box was recorded. With the gap position a relative movement of an ion in the gap was calculated. The flux of ions from the liquid to the ablated layer was calculated as follows: A value of +1 was assigned if a positive ion moved by more than half of the gap length to the right during the simulation and -1 if the charge of the ion was negative. A movement of a positive ion in the other direction by more than half of the gap was counted as -1 and accordingly for a negative ion as +1 . For all ions in each trajectory the corresponding fluxes were summed up to a total flux and rounded up to the nearest integer at the end of the simulation to account for the fact that only whole charges could be transfered.

In the lower panel of Figure 4.11a,b the average flux for all trajectories with the charge of the layer at the beginning $\Delta q$ is shown. At a concentration of $15 \mathrm{mM}$ almost no flux is observed because ions were hardly found in the gap. In contrast at $154 \mathrm{mM}$ a flux is seen which increases with $\Delta q$ and was directed against the charge of the layer leading to a neutralization. This flux narrows the distribution observed at the beginning to the end distribution during the simulation time of 100 ps. 


\subsubsection{Isolation of Biomolecules from Liquid Water Matrix}

Since the ablation of layers induced by a shock wave results in charged layers, isolation of molecules from the liquid water matrix carrying a net charge and their detection by mass spectrometry should also be feasible. The experimental setup is able to yield high resolution mass spectra of fragile biomolecules like cytochrom c, hemoglobin or insulin. This possibility makes this analysis method to a very soft technique in comparison to others like electrospray ionization or electron impact ionization for the analysis of biomolecules.

In order to quantify the softness of the technique, simulations as described before were performed with an arginine molecule at the right end of the equilibrated box. This simulation resulted also in the ablation of layers, which contained arginine surrounded by water (Fig. 4.12). In the experimental setup the surrounding water is evaporated on the way to the mass spectrometer on a time scale of $\mu$ s and the arginine molecule ends up with a charge, to be detected by mass spectrometry. Although dissociation of the

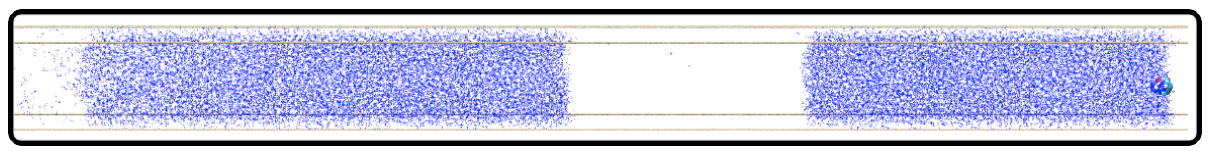

Figure 4.12: Snapshot at 100 ps of the ablation process of arginine.

molecule could not be accounted for in the simulation since the atoms are kept together by harmonic oscillators, the increase in temperature in the ablated layer can be used to study the present conditions upon ablation. To this end, the temperature of all water molecules in the ablated layer was corrected with the kinetic energy corresponding to the center of mass movement of the whole layer. The temperature after the ablation increased only by $40 \mathrm{~K}$. This small increase and the subsequently evaporating cooling, 
which was not considered in the simulations and would decrease the temperature anew, explain the softness of the experimental analysis method for biomolecules: Biomolecules remain always in their natural environment suffering only a small increase in temperature and the impact of the shock wave, which lasts very shortly.

First studies on the change of the structure of whole proteins was investigated for hemoglobin by Frank Wiederschein [71]. After the ablation an increase in the root mean square deviation of the backbone atom positions by $0.4 \mathrm{~nm}$ was observed, however, the molecule stayed intact for the simulation whole time of $135 \mathrm{ps}$ and was ablated in a layer surrounded by water as in the case of arginine. This results represents evidence that biomolecules overcome the impact of the shock wave without suffering mayor structural changes. 


\section{Chapter 5}

\section{Discussion}

This Chapter compares the simulation results with experimental data. Dynamical and structural properties of metastable water will be related to characteristic changes in measured photoelectron spectra. Furthermore, the desorption and charge mechanism enabling the mass spectrometrical analysis of large biomolecules like proteins will be elucidated.

\subsection{Ultrafast Dynamics in Metastable Liquids}

The evolution of metastable liquids shows characteristic features, which were investigated experimentally by time resolved photoelectron spectroscopy. Via an IR-XUV pump probe technique a liquid beam in vacuum was heated up to different temperatures, and the dynamics was followed through changes in characteristic bands of the photoelectron spectra. The dynamics was studied experimentally for water and methanol.

With the simulation results the measured dynamics for metastable water could be separated into two temperature regimes, in good agreement with the experiment. 
The first regime is characterized by temperatures lower than the critical temperature and the second by temperatures above $\left(T_{\text {crit }}=647 \mathrm{~K}\right)$. On the basis of the simulation results the dynamics of metastable water at subcritical temperatures can be described as the evaporation of single molecules. These molecules leave the liquid phase at the liquid-vacuum interface, when they have obtained enough kinetic energy to overcome the free energy barrier between the liquid and the gas phase (Fig. 3.11). In the measured photoelectron spectra (Fig. 3.7) as well as in the simulation the process is slow $(t \sim 200 \mathrm{ps})$.

For metastable water at supercritical conditions the measured spectra revealed faster dynamics of the metastable liquid and the formation of a new band in the photoelectron spectra after $\sim 100$ ps. The simulations showed a dispersion of the liquid into single molecules and aggregates, which was accompanied by a change in the nature of the hydrogen bonds (Fig. 3.9). Furthermore, the new measured band in the spectra were identified as aggregates built out from 5-20 molecules (Fig. 3.16).

Methanol at supercritical and subcritical conditions, in contrast, did not form any aggregates, and the evolution of the metastable liquid took place via the evaporation of single molecules only. This is in agreement agrees with the experiment, where no new band at supercritical temperatures displaying aggregates was observed, and the dynamics were much slower (Fig. 3.8). An analysis of the hydrogen bond kinetics of water and methanol at standard conditions $(300 \mathrm{~K}, 1$ bar) and at supercritical high density conditions revealed that the formation and dissociation of hydrogen bonds is a factor of four slower in methanol than in water at standard conditions and a factor of two at supercritical conditions. Since the first step in the evolution of the metastable liquids is the dissociation and formation of hydrogen bonds, the different time scale for this process in methanol compared to water explains the slower dynamics of methanol. Furthermore, this faster formation of hydrogen bonds may explain why aggregates were observed at supercritical conditions for water and not for methanol. 


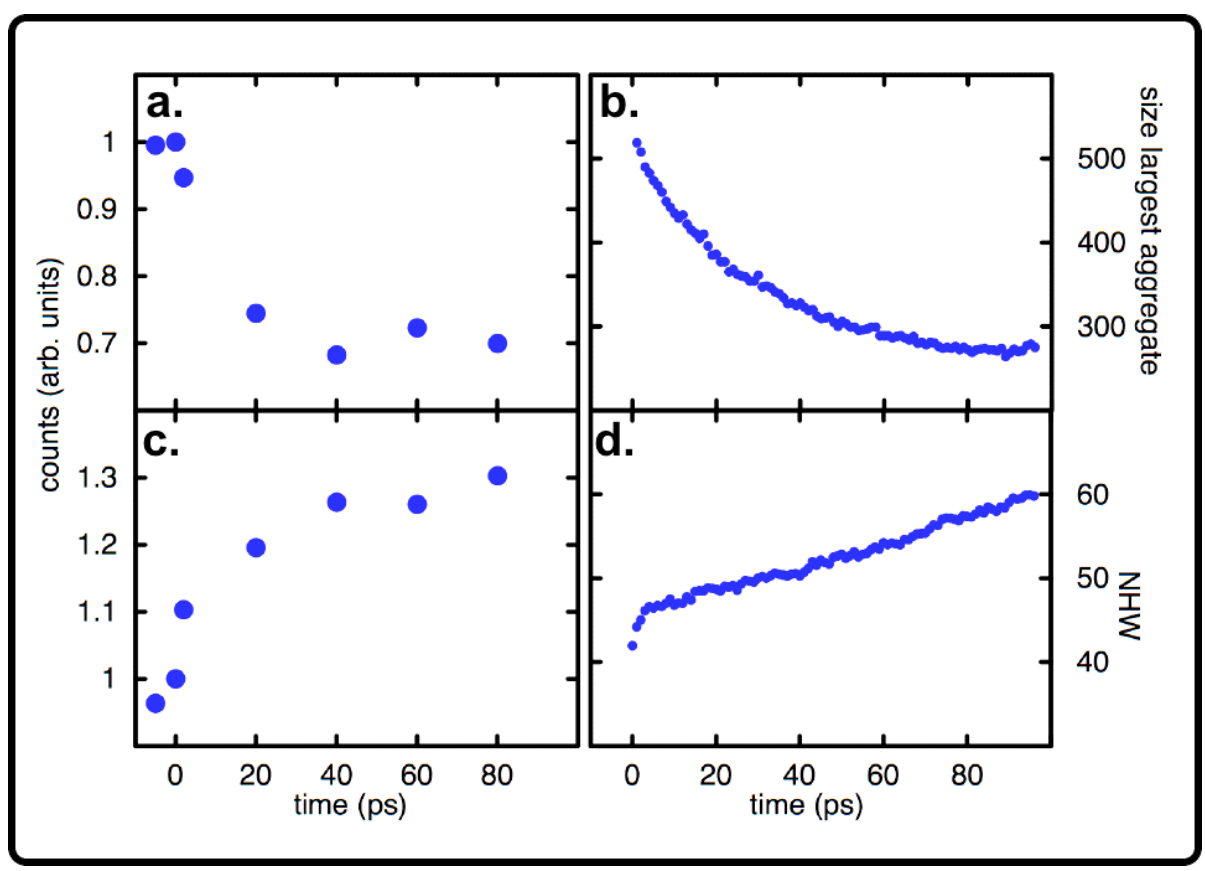

Figure 5.1: Measured intensities at characteristic electron binding energies in photoelectron spectra with an IR-wavelengths of $2900 \mu \mathrm{m}$ as a function of time (a. maximum of the liquid band at $11.16 \mathrm{eV}$ b. maximum of the gas band $12.6 \mathrm{eV}$ ). c.) Average size of the aggregates larger than 200 molecules in the simulation of supercritical water. d.) Fraction of not hydrogen bonded molecules in the same simulations.

Figure 5.1 compares the evolution of characteristic electron binding energies in the measured photoelectron spectra of supercritical metastable water to the dynamics in the simulations. Figure 5.1a shows the measured intensity of the electrons with a binding energy characteristic for the liquid phase $\left(\mathrm{E}_{\mathrm{bind}}=11.16 \mathrm{eV}\right)$ as a function of time, and Figure $5.1 \mathrm{~b}$ the average size of aggregates larger than 200 molecules in the simulation. It can be seen that the dynamics of the simulations in the first $100 \mathrm{ps}$ match the experimental one. Agreement between experiment and simulation is also found for photoelectrons with binding energies representing the gas phase $\left(\mathrm{E}_{\mathrm{bind}}=12.6 \mathrm{eV}\right)$ (Fig. 5.1c) and the change in the fraction of non-hydrogen bonded molecules in the simulation (Fig. 5.1d). Note that for comparison 


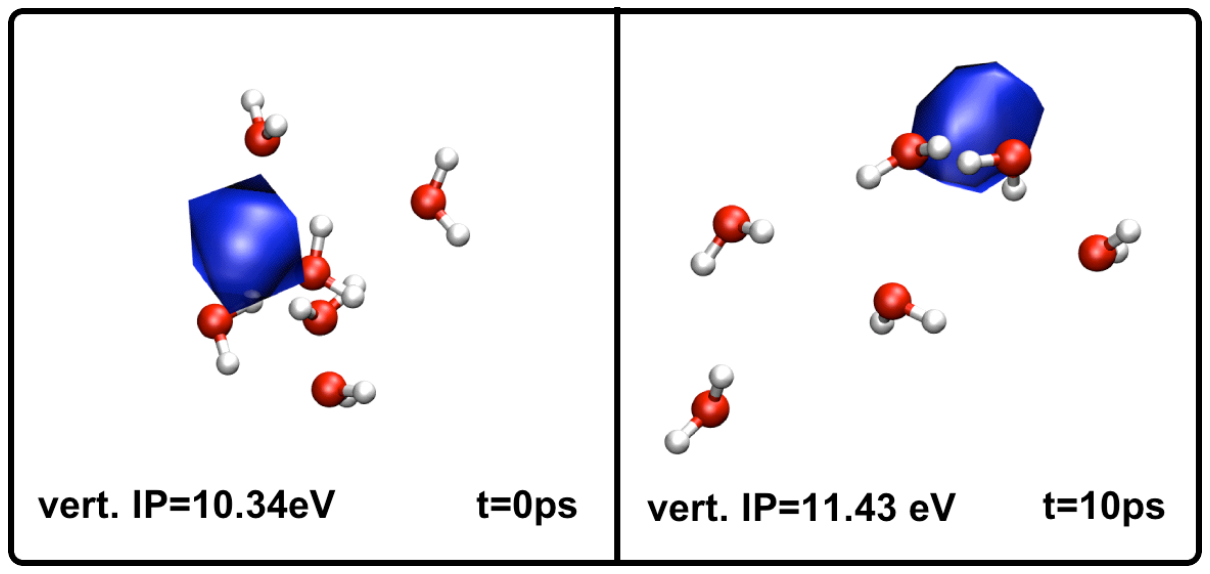

Figure 5.2: Orbital surfaces (isovalue $-0.019 \mathrm{e} / \AA^{3}$ ) of the highest occupied molecular orbital in the simulated ensembles at 0 and $10 \mathrm{ps}$ from the dynamics of supercritical metastable water with the respective ionization potentials.

the first picoseconds in the experiment can not be taken into account, since this time period represents the formation of the metastable phase and is not present in the simulation.

To address the shifts observed in the band representing the liquid phase of the photoelectron spectra (Fig. 3.7), QM/MM calculations on snapshots obtained from the supercritical water simulations were performed. These calculations yielded distribution of electron binding energies with the same shift to larger binding energies in the first $10 \mathrm{ps}$ was observed for the electrons representing the liquid phase in the measured spectra (Fig. 3.18). Additionally, the main contributions to these shifts in the ionization potentials of an electron in the highest occupied molecular orbital (HOMO) were analyzed (Fig. 3.19 upper panel). The shifts were not only induced through a stabilization of the ionized state in the condensed phase, but also by the stabilization of the neutral molecules representing the ground state of the ionization process (Fig. 3.19). The stabilization of the neutral and ionized state were attributed to the presence of hydrogen bonds.

Thus, QM/MM calculations are able to display changes in orbital energies 
during chemical reactions like the dissociation and formation of hydrogen bonds. These changes can be verified experimentally with time resolved photoelectron spectroscopy. As an example of the observed changes in the orbitals Figure 5.2 shows the surface of the HOMO with the lowest ionization potential of the ensemble at the beginning of the simulation in comparison to the one after $10 \mathrm{ps}$. At 0 ps the orbital is spanned over three hydrogen bonded water molecules, whereas after $10 \mathrm{ps}$ hydrogen bonds between the water molecules are broken and the orbital becomes more localized. This increasing localization of the orbital on one molecule accompanied with an increase in the vertical ionization potential during the simulation is correlated to the shift of the whole measured photoelectron spectrum to larger binding energies.

In summary, Molecular Dynamics simulation provided a microscopic picture of the evolution of a metastable liquid. Additionally, QM/MM calculation were able to correlate changes in the measured photoelectron spectrum to the evolution of orbitals on a microscopic scale.

\subsection{Laser Induced Isolation of Charges from Water}

The isolation of charges such as ions or charged molecules from matrices represents one of the greatest challenges for mass spectrometrical (MS) analysis. Laser Induced Liquid Beam Ionization Desorption (LILBID) is a new technique, in which molecules are isolated via an IR-Laser from a water filament in vacuum. In comparison to other MS-techniques, this analysis method allows to identify fragile molecules like proteins and protein complexes in mixtures and, additionally, to quantify their concentrations in solution. 


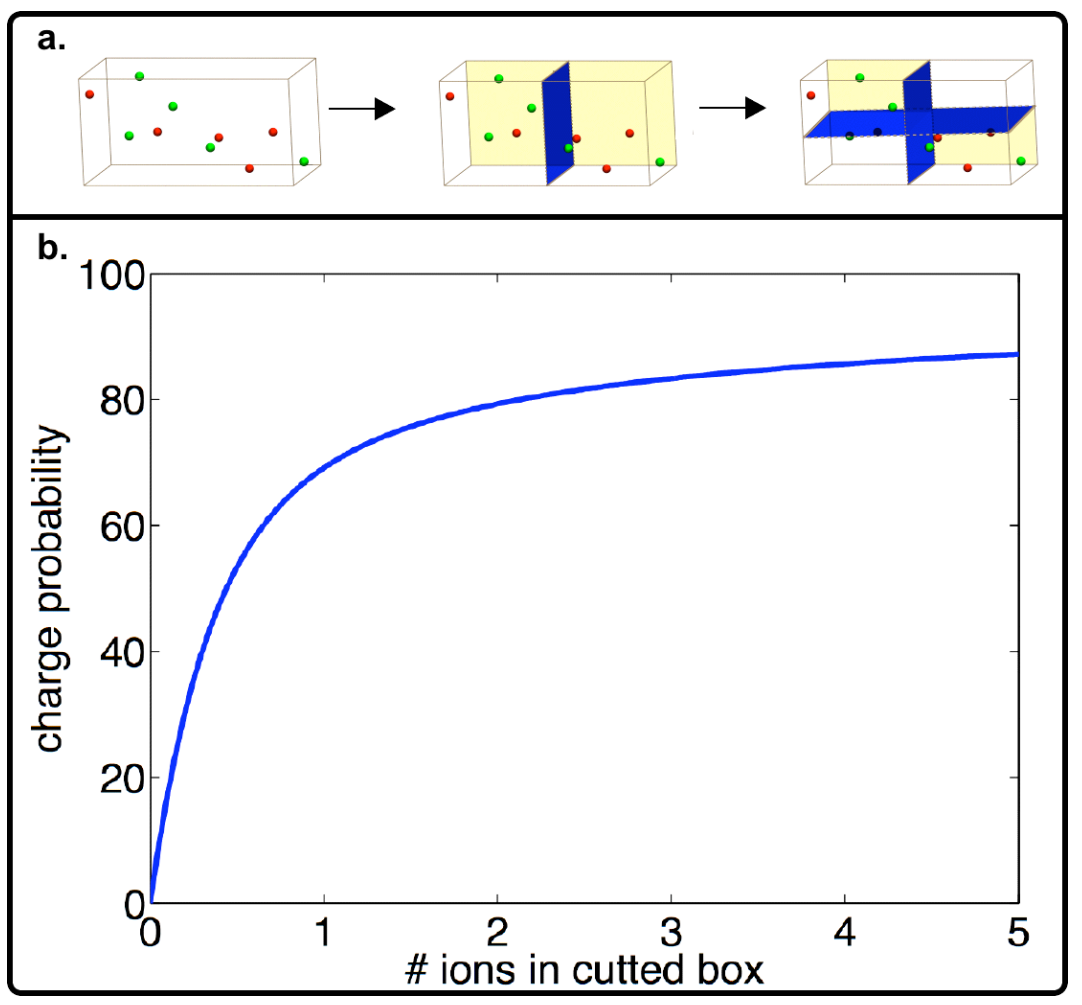

Figure 5.3: a.) Scheme illustrating the creation of charged boxes with unequal number of ions by cutting the original neutral box in smaller boxes (charged boxes have yellow background). b.) Charge probability to obtain a charged box by cutting boxes containing in average $n$ number of ions from an arbitrary volume assuming a poisson distribution for the number of ions.

However, little is known about the desorption process and the charging mechanism. Here, Molecular Dynamics (MD) simulations were performed in which a temperature gradient in water was used to mimic the absorption of the laser radiation by the liquid filament in the experiment. During the simulations shock waves lead to the ablation of water layers and droplets from the liquid phase (Fig. 4.4). The small increase in temperature of the ablated layers explains why the LILBID-technique is able to isolate fragile proteins without fragmentation. 
Simulations performed with sodium and chloride ions at two concentrations, 15 and $154 \mathrm{mM}$, showed that some of the ablated layers carried a net charge, arising from an unequal number of positive and negative ions. To explain the distribution of layers with a net charge $q$ a simple model was developed.

Starting from a large volume with the same number of positive and negative ions the shock wave, as shown in the simulation results, would disperse this volume into small subvolumes in a fast process $(t \sim 20 \mathrm{ps})$. This procedure is shown schematically in Figure 5.3a. Some of the subvolumes will exhibit an unequal number of positive and negative ions and, thus, be charged (charged subvolumes are displayed with a yellow background). If the size of the subvolumes is reduced further the total number of charged ones decreases.

The probability to obtain a charged box after dividing the original volume depends on the expected number of ions per divided box. A large number of ions increases the probability to obtain a charged box (see Fig. 5.3b). Thus, this probability also depends on the ion concentration in the original box before dividing it into subvolumes. Higher concentrations would lead to larger probabilities at a given size of the subvolumes.

More quantitatively, this probability equals the expectation value $k$ for a discrete variable which has a known mean value $\lambda$. This value is given by the Poisson distribution (see eq. 4.5). Assuming the number of positive and negative ions in the divided box follow this distribution, the maximum $\lambda$ is given as the expected number of ions per divided box, which is calculated from the ion concentration and the volume of the box. If the distribution of the positive and the negative ions are independent from each other, the charge probability to obtain a divided box with charge $q$ can be calculated analytically via equation 4.7. The resulting total probability to find charged boxes as a function of average number of ions in the box is shown in Figure $5.3 \mathrm{~b}$.

However, the simulation results at different ion concentrations revealed 
Figure 5.4: Snapshot at 60 ps from a MD-simulation showing the ions between the remaining liquid and the ablated layer which contribute to the flux during the ablation at a concentration of $154 \mathrm{mM}\left(\mathrm{Na}^{+}\right.$-ions are displayed red and $\mathrm{Cl}^{-}$-ions green. Two periodic images are shown).

three different regimes for the charge probability of the ablated layers, which depend on the ion concentration.

In the first regime, where ion concentrations are smaller than $10^{-3} \mathrm{M}$, the charge probability can be calculated via equation 4.7, as described in the simple model above. For ion concentrations between $10^{-3} \mathrm{M}$ and $10^{-1} \mathrm{M}$, which renders the second regime, Monte-Carlo (MC) simulations displayed a deviation from the simple model. In these MC simulations boxes at varying ion concentrations were first equilibrated with a continuum solvent model and then these boxes were divided into subvolumes. The inclusion of the ion-ion interactions in the simulations reduced the probability to obtain a charged subvolume, especially for the boxes with high charged states (see Fig. 4.9). The third regime applies for concentration larger than $10^{-1} \mathrm{M}$. Molecular Dynamics simulations of the ablation process at $154 \mathrm{mM}$ revealed that the distribution of charged layers at the beginning, which, in principle, is represented through the second regime, changed during the simulation. The reason for the change was found to be a flux of ions during the formation of the ablated layers (see Fig. 5.4). This flux of ions increases with the absolute charge of the layer at the beginning of the ablation process and compensates its net charge (see Fig. 4.11). Because of the neutralization of the layers by the flux the probability to obtain a charge layer is decreased even further for concentration higher than $10^{-1} \mathrm{M}$.

The three regimes for the generation of charges from a liquid were tested experimentally by measuring mass spectra of $\mathrm{NaCl}$-water solutions at dif- 


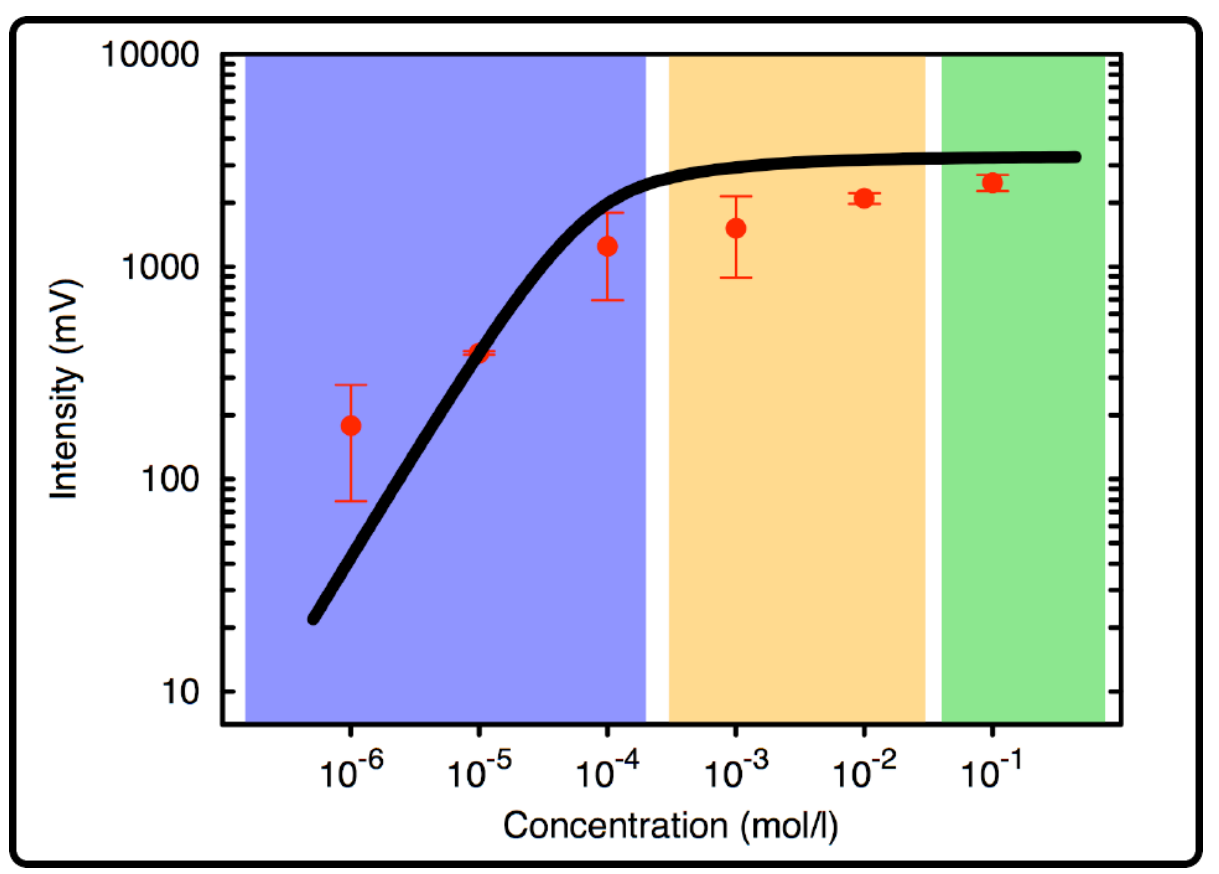

Figure 5.5: Summed intensity over all aggregates containing $\mathrm{Na}^{+}-$ions in the mass spectrum measured from different salt solutions with the LILBID-MS technique. The colors indicate which charge mechanism can be applied. In the blue region a Poisson distribution can be assumed, in the orange region the interaction of the ions decreases the probability predicted by the Poisson distribution and the green region represents the region where the ion flux in the interface between remaining liquid and ablated layer during the ablation process neutralize the ablated layer reducing the charge probability even further. The black line displays the intensity calculated with equation 5.1 for an average droplet size of $13.6 \mathrm{~nm}$.

ferent concentrations with the LILBID technique. Figure 5.5 shows the intensity summed over all aggregates in the mass spectrum containing $\mathrm{Na}^{+}$ (see Fig. 4.2) at different ion concentrations. This increasing intensity with ion concentration reflects the number of $\mathrm{Na}^{+}$aggregates, which carried nonzero charge after the desorption from the liquid matrix.

To apply these three regimes to the experimental data the size of the droplets in the experiment is needed. The average size of the layers obtained in the MD simulations can not be used to obtain the droplet volume, 
since the layers have periodic boundary conditions in two dimensions, which prevents them to form droplets due to the unfavorable volume to surface ratio. However, the simulations at the two ion concentrations revealed only small changes in the average layer length. Therefore, the volume of the droplets in the experiment is assumed to be constant over the measured concentration range.

In accordance to the first regime, for concentrations smaller than $10^{-3} \mathrm{M}$ the measured intensities $I(c)$ can be calculated analytically assuming a poisson distribution of ions. The intensities are given by the following equation

$$
I(c)=P\left(c V N_{A}\right) I_{\max },
$$

where $P\left(c V N_{A}\right)$ is the probability to get a charged droplet with volume $V$ from a solution at concentration $c$ and $N_{A}$ the Avogadro number. $I_{\max }$ corresponds to the intensity, which would be reached if all droplets are charged. MD-simulations of the ablation process at $154 \mathrm{mM}$ showed that the deviation of the charge probability due to the flux and the ion-ion interaction is approximately $75 \%$ from the predicted value. Assuming that at a concentration of $10^{-1} \mathrm{M}$ the predicted probability would be near $100 \%$, $I_{\max }$ can be estimated by dividing the measured intensity with the correction factor of $75 \%\left(I_{\max }=3300 \mathrm{mV}\right)$.

The black line in Figure 5.5 shows a fit of equation 5.1 with this value up to a concentration of $10^{-4} \mathrm{M}$ to the measured intensities. The obtained average volume for the ablated droplets is $10.5 \cdot 10^{3} \mathrm{~nm}^{3}$ corresponding to an average radius of $13.6 \mathrm{~nm}$. It should be noted that other values of $I_{\max }$ lead only to minor changes in the properties of the droplets. The measured intensities at $10^{-3} \mathrm{M}$ and $10^{-2} \mathrm{M}$ are smaller than the fitted values, since the ion-ion interaction reduces the probability of obtaining a charged droplet. Considering the experimental conditions after the ablation of the droplets, their obtained size is reasonable. The droplets have to travel from the water filament to the detector through the vapor phase surrounding the liquid 
filament in $\sim 10 \mu \mathrm{s}$. On the way they loose most of their water molecules via collisions with gas phase molecules and evaporation. When they reach the detector, only some water molecules are left, as can be seen in the mass spectra.

The dispersion of the liquid explains the generation of charges during the measurement of $\mathrm{NaCl}$-solution in LILBID-MS. For the desorption of biomolecules, however, the charge mechanism may vary from the latter one since changes in the protonation states of the titrable amino acids during the desorption may also influence the overall charge of the molecule.

Apart from lasers, dispersion of liquids containing ions can also be achieved via strong impacts on solid surfaces or particles. Some of the divided volumes have a probability to possess a net charge after the impact, which can be derived from the proposed charge mechanism.

First evidences for the generation of charges upon impact are obtained from water clusters, which were accelerated in vacuum on solid surfaces. The fragments built after the impact often exhibit a charge [23]. The creation of charge fragments may rise from the fast division of the neutral clusters into smaller ones upon impact. At low ion concentrations the charge probability could be calculated from the ion concentration of the clusters at the beginning and the size of the fragments.

In thunderstorms charges are also separated, which result in large electric fields between the clouds and the earth surface. These large electric fields become visible as lightnings, exhibiting discharges in the order of $20 \mathrm{kA}$. For the charge generation in the clouds several mechanism have been proposed, which rely on the electric field between the ionosphere and the earth surface or on the uptake of charges from the surface of ice particles.

The proposed mechanism may point to a new way how charges can be generated. Liquid water droplets in clouds hit ice particles, and are split into smaller droplets. If the droplets contained ions, there is a probability that some of the smaller ones have a net charge, rising from an unequal 
distribution of ions.

Field studies throughout the Untied States reported $\mathrm{NaCl}$ concentrations in the atmosphere, which vary over a wide range displaying a maximum $\mathrm{NaCl}$ concentration of $\sim 10^{-3} \mathrm{M}$ at the coastal area [74]. This low concentration allows the assumption of a Poisson distribution for positive and negative ions and with the knowledge of the average droplet size after the impact on an ice particle the fraction of charged droplets can be calculated. Therefore, the proposed model can represent an alternative charge mechanism for the generation of the electric fields in thunderstorms. 


\section{List of Figures}

2.1 Scheme for the QM/MM-embedding. . . . . . . . . . . 21

2.2 Water models . . . . . . . . . . . . . . . . 25

2.3 OPLS methanol model . . . . . . . . . . . . . 27

3.1 Experimental setup for ultrafast dynamics of metastable liquids 34

3.2 Liquid beam and nozzle . . . . . . . . . . . . . . 35

3.3 Scheme: Basic principles of photoelectron spectroscopy . . . 37

3.4 Spectrum of gas phase water and molecular orbitals of the water monomer . . . . . . . . . . . . . . 40

3.5 Scheme: Solvent influences on ion and neutral molecule . . . 41

3.6 Photoelectron spectrum of gas phase and liquid water . . . . 45

3.7 Sequence of photoelectron spectra of water for different deposited energies . . . . . . . . . . . . . . . . 4 47

3.8 Sequence of photoelectron spectra of methanol for different deposited energies . . . . . . . . . . . . . . 48

3.9 Snapshots of dynamics of metastable water at supercritical conditions . . . . . . . . . . . . . . . . . 51

3.10 Phase diagram of water . . . . . . . . . . . . . . . . 52

3.11 Snapshots of dynamics of metastable water at subcritical conditions . . . . . . . . . . . . . . 52

3.12 Snapshots of dynamics of methanol at different temperatures 53

3.13 Average density as a function of time of water simulations preformed with the SPC water model at different temperatures 55 


\section{List OF FigURES}

3.14 Average density as a funtion of time of water simulations for different water models . . . . . . . . . . 56

3.15 Hydrogen bond dynamics of water and methanol for different temperatures . . . . . . . . . . . . . . . . 5 58

3.16 Distribution of aggregates in supercritical water simulations 61

3.17 Lifetimes of dimer, trimer and tetramer . . . . . . . . . . 63

3.18 Simulated photoelectron spectra for supercritical water at different times during the expansion process . . . . . . . . 66

3.19 Stabilization energies of the ground and the ionized state of the six water molecules in the QM region for different times during the expansion process for supercritical metastable water and vertical ionization energies for the electrons in the HOMO-orbitals . . . . . . . . . . . . . . 67

4.1 Experimental setup for the isolation of charged molecules from liquid water . . . . . . . . . . . . . 70

4.2 Mass spectra of NaCl-solutions at different concentrations . 71

4.3 Kinetic energy gradients along the water box . . . . . . . . 74

4.4 Snapshots of desorption process . . . . . . . . . . 75

4.5 Density profile during the desorption process along the box . 77

4.6 Dependence of the velocity of the shock wave on the deposited energy . . . . . . . . . . . . . . . 79

4.7 Poisson distribution for $\lambda=1.78$ and 17.8. . . . . . . . . 81

4.8 Relative occurrence for layers at the end with charge $\Delta q$ obtained from the simulations and the predicted probability by a Poisson distribution of ions . . . . . . . . . . 83

4.9 Relative occurrence for layers with charge $\Delta \mathrm{q}$ obtained from Monte Carlo simulations and the predicted probability by a Poisson distribution of ions at ion concentration of 1.5, 15 and $154 \mathrm{mM} \ldots \ldots \ldots \ldots \ldots$ 
4.10 Comparison of the distributions of charged layers before equilibration and after equilibration . . . . . . . . . 86

4.11 Relative occurrence for layer at the beginning and at the end with charge $\Delta q$ obtained from the simulations and the flux for simulation with layers with charge $\Delta q$ at the beginning . 87

4.12 Ablation of arginine . . . . . . . . . . . . . . . 89

5.1 Measured intensities at characteristic electron binding energies in PE-spectra as a function of time in comparison to size of the largest aggregate and the fraction of not hydrogen bonded molecules in the simulations . . . . . . . . . . . . . 93

5.2 Highest occupied molecular orbital of the simulated ensembles at 0 and 10ps during the transition of supercritical metastable water into vacuum with the respective ionization potentials . 94

5.3 Mechanism for the creation of charge droplets by cutting a given volume in smaller boxes . . . . . . . . . . . . 96

5.4 Snapshot at $60 \mathrm{ps}$ from a MD-simulation showing the ions which contribute to the flux during the ablation of a layer at a concentration of $154 \mathrm{mM} \ldots \ldots$. . . . . . . . . . . . 98

5.5 Summed intensity over all aggregates containing $\mathrm{Na}^{+}$-ions in the mass spectrum measured from different salt solutions with the LILBID-MS technique . . . . . . . . . . . . . . . 



\section{List of Tables}

2.1 Thermodynamic properties of the SPC- and the TIP4P model 26

2.2 Geometrical properties of OPLS methanol monomer . . . . . 28

2.3 Thermodynamic properties of OPLS methanol model . . . . 28 



\section{Bibliography}

[1] E. Voehringer-Martinez, B. Hansmann, H. Hernandez, J. S. Francisco, J. Troe and B. Abel, Science, 2007, 315 (5811), 497-501.

[2] M. L. Cowan, B. D. Bruner, N. Huse, J. R. Dwyer, B. Chugh, E. T. J. Nibbering, T. Elsaesser and R. J. D. Miller, Nature, 2005, 434, 199.

[3] P. Wernet, D. Nordlund, U. Bergmann, M. Cavalleri, M. Odelius, H. Ogasawara, L. A. Näslund, T. K. Hirsch, L. Ojamäe, P. Glatzel, L. G. M. Pettersson and A. Nilsson, Science, 2004, 304, 995-999.

[4] P. Ball, Nature, 2008, 452 (7185), 291-292.

[5] P. Poole, F. Sciortino, U. Essmann and H. E. Stanley, Nature, 1992, 360 (6402), 324-328.

[6] P. Gallo, M. Minozzi and M. Rovere, Physical Reviews E, 2007, 75.

[7] N. Akiya and P. Savage, Chem Rev, 2002, 102 (8), 2725-2750.

[8] P. Wernet, D. Testemale, J. Hazemann, R. Argoud, P. Glatzel, L. Pettersson, A. Nilsson and U. Bergmann, Journal of Chemical Physics, 2005, 123.

[9] B. Winter, E. F. Aziz, U. Hergenhahn, M. Faubel and I. V. Hertel, Journal of Chemical Physics, 2007, 126, 124504.

[10] H. Siegbahn, J.Phys.Chem., 1985, 89, 897. 
[11] O. Link, Femtosekunden Photoelektronenspektroskopie mit extrem starker ultravioletter Strahlung an Flüssigkeitsgrenzflächen, Dissertation, 2007.

[12] B. L. Holian, Phys Rev A, 1988, 37 (7), 2562-2568.

[13] B. L. Holian, W. G. Hoover, B. Moran and G. K. Straub, Phys Rev A, 1980, 22 (6), 2798-2808.

[14] R. Knochenmuss and L. Zhigilei, Journal of Physical Chemistry B, 2005, 109 (22), 947.

[15] Y. Dou, N. Winograd, B. Garrison and L. Zhigilei, Journal of Physical Chemistry B, 2003, 107 (10), 2362-5.

[16] Z. Chen, A. Bogaerts and A. Vertes, Journal of Chemical Physics, 2006, 89, 041503.

[17] L. Zhigilei, E. Leveugle, B. Garrison, Y. Yingling and M. Zeifman, Chemical Reviews, 2003, 103 (2), 321-348.

[18] M. Karas and R. Kruger, Chemical Reviews, 2003, 103 (2), 427-40.

[19] A. Vogel and V. Venugopalan, Chemical Reviews, 2003, 103 (2), 577644.

[20] B. Abel, A. Charvat, U. Diederichsen, M. Faubel, B. Girmann, J. Niemeyer and A. Zeeck, International Journal of Mass Spectrometry, 2005, 243 (2), 177-188.

[21] A. Charvat, B. Stasicki and B. Abel, Journal of Physical Chemistry A, 2006, 110 (9), 3297-3306.

[22] J. Latham and B. Mason, Proc R Soc Lon Ser-A, 1961, 260 (130), 537.

[23] C. Gebhardt, H. Schroder and K. Kompa, Nature, 1999, 400 (6744), 544-547.

[24] M. Born and R. Oppenheimer, Ann. d Physik, 1927, 84, 457-484. 
[25] F. Daan and B. Smit, Understanding Molecular Simulation, 1996.

[26] L. Verlet, Physical Reviews B, 1967, 34, 1311-1327.

[27] S. Attila and O. Neil, Modern Quantum Chemistry, 1989.

[28] J. Sauer and M. Sierka, Journal of Computational Chemistry, 2000, 21, 1470 .

[29] J. Gao, Acc. Chem. Res, 1996, 29, 298.

[30] M. Field, P. Bash and M. Karplus, Journal of Computational Chemistry, 1990, 11, 700.

[31] R. Friesner and V. Gaullar, Annu. Rev. Phys. Chem., 2005, 56, 389.

[32] A. Warshel and M. Levitt, Journal of Molecular Biology, 1976, 103, 227.

[33] D. van der Spoel, E. Lindahl, B. Hess, G. Groenhof, A. E. Mark and H. J. C. Berendsen, Journal of Computational Chemistry, 2005, 26, $1701-1718$.

[34] W. van Gunsteren, S. Billeter, A. Huenenberger, P. Krueger, A. Mark, W. Scott and I. Tironi, Biomolecular simulation: GROMOS96 manual and user guide:, 1996.

[35] W. Jorgensen and J. Tirado-Rives, Journal of the American Chemical Society, 1988, 110, 1657.

[36] A. MacKerell, Journal of Physical Chemistry B, 1998, 102, 3586.

[37] J. Wang, P. Cieplak and P. Kollman, Journal of Computational Chemistry, 2000, 21, 1049.

[38] R. Bukowski, K. Szalewicz, G. C. Groenenboom and A. van der Avoird, Science, 2007, 315, 1249.

[39] B. Guilliot, Journal of Molecular Liquids, 2002, 101, 219-260.

[40] J. D. Bernal and R. H. Fowler, Journal of Chemical Physics, 1933, 1, 515. 
[41] F. Stillinger and A. Rahman, Journal of Chemical Physics, 1974, 60, 1545 .

[42] H. J. C. Berendsen, J. P. M. Postma, W. F. van Gunsteren and J. Hermans, Intermolecular Forces, 1981.

[43] W. L. Jorgensen, J. Chandrasekhar, J. D. Madura, R. W. Impey and M. Klein, Journal of Chemical Physics, 1983, 79, 926-935.

[44] M. Mahoney and W. Jorgensen, Journal of Chemical Physics, 2000, 112, 8910-8922.

[45] S. Rick, S. Stuart and B. Berne, Journal of Chemical Physics, 1994, 101, 6141-6156.

[46] H. J. C. Berendsen, J. R. Grigera and T. P. Straatsma, Journal of Chemical Physics, 1987, 91, 6269.

[47] W. Jorgensen, Journal of the American Chemical Society, 1981, 103, 335.

[48] W. Jorgensen, Journal of Physical Chemistry, 1986, 90, 1276.

[49] T. Yamaguchi, C. J. Benmore and A. K. Soper, Journal of Chemical Physics, 2000, 112, 8976.

[50] M. Chalaris and J. Samios, Journal of Physical Chemistry B, 1999, 103, 1161.

[51] E. Espinosa, E. Molins and C. Lecomte, Chemical Physics Letters, 1998, 285, 170-173.

[52] A. Luzar and D. Chandler, Nature, 1996, 379, 55-57.

[53] A. Luzar, Journal of Chemical Physics, 2000, 113, 10663.

[54] A. Luzar and D. Chandler, Physical Reviews Letters, 1996, 76 (6), 928-931.

[55] D. van der Spoel, P. J. van Maaren, P. Larsson and N. Tomneanu, Journal of Physical Chemistry B, 2006, 110, 4393-4398. 
[56] M. Faubel, S. Schlemmer and J. P. Z. Toennies, Z. Phys. D, 1988, 10, 269.

[57] M. Faubel and T. Kisters, Nature, 1989, 339, 527.

[58] B. Winter and F. Manfred, Chemical Reviews, 2006, 106, 1176-1211.

[59] H. Hertz, Ann. Physik, 1887, 31, 983.

[60] A. Einstein, Ann. Phys., 1905, 17, 132.

[61] I.-F. W. Kuo and C. J. Mundy, Science, 2004, 303, 658.

[62] H. Agren and H. Siegbahn, Journal of Chemical Physics, 1984, 81, 488.

[63] H. Agren and V. Carravetta, Molecular Physics, 1985, 55, 901.

[64] S. Myneni, Y. Luo, Laslund, M. Cavalleri, L. Ojamäe, H. Ogasawara, A. Pelmenschikov, P. Wernet, P. Vaterlein, C. Heske, Z. Hussain, L. G. M. Pettersson and A. Nilsson, Journal of Physics: Condensed Matter, 2002, 14, 213-219.

[65] H. J. C. Berendsen, J. P. M. Postma, A. DiNicola and J. R. Haak, Journal of Chemical Physics, 1984, 81, 3684-3690.

[66] T. Darden, D. York and L. Pedersen, Journal of Chemical Physics, 1993, 98, $10089-10092$.

[67] C. A. Jeffrey and P. H. Austin, Journal of Chemical Physics, 1999, 110, 484-496.

[68] L. Belau, K. R. Wilson, S. R. Leone and A. Musahid, Journal of Physical Chemistry A, 2007, 111, 10075-10083.

[69] A. Charvat, A. Bögehold and B. Abel, Australian journal of chemistry, 2006, 59 (2), 81-103.

[70] A. Beinsen, Flüssigstrahlmassenspektrometrie: Oxidationskinetik von Biomolekülen und Ionenanalytik in wässrigen Systemen, Diplomarbeit, 2006. 
[71] F. Wiederschein, to be published, Dissertation, 2008.

[72] F. Sciortino and S. Sastry, Journal of Chemical Physics, 1994, 100 (5), 3881-3893.

[73] J. Walsh and M. Rice, Journal of Chemical Physics, 1957, 26 (4), 815-823.

[74] J. Collett, A. Bator, D. Sherman, K. Moore, K. Hoag, B. Demoz, X. Rao and J. Reilly, Atmos Res, 2002, 64 (1-4), 29-40. 


\section{Lebenslauf}

\section{Persönliche Daten}

Esteban Vöhringer-Martinez

Lichtenbergstr. 1

37075 Göttingen

Tel.: (05 51) 3072733

E-Mail: esteban.voehringer@chemie.uni-goettingen.de

Geboren am 02.09. 1978 in Córdoba, Argentinien

verheiratet, deutsch

\section{Schulbildung}

03/1985-03/1990 Grundschule in Córdoba, Argentinien

03/1990-06/1999 Hochschulreife am Goethe-Gymnasium in Emmendingen (Leistungskurse Mathematik und Physik)

\section{Zivildienst}

07/1999-07/2000 Zivildienst im Landeskrankenhaus Göttingen

\section{Studium}

10/2000-10/2002 Chemie Vordiplom an der Universität Göttingen

10/2002-02/2005 Chemie Diplom an der Universität Göttingen 